\title{
Offset-Based Beamforming: A New Approach to Robust Downlink Transmission
}




\title{
OFFSET-BASED BEAMFORMING: A NEW APPROACH TO ROBUST DOWNLINK TRANSMISSION
}

\author{
BY \\ MOSTAFA MEDRA, B.Sc., M.Sc.
}

\begin{abstract}
A THESIS
SUBMITTED TO THE DEPARTMENT OF ELECTRICAL \& COMPUTER ENGINEERING AND THE SCHOOL OF GRADUATE STUDIES OF MCMASTER UNIVERSITY
\end{abstract}

IN PARTIAL FULFILMENT OF THE REQUIREMENTS

FOR THE DEGREE OF Doctor of Philosophy

(c) Copyright by Mostafa Medra, September 2017

All Rights Reserved 
Doctor of Philosophy (2017)

(Electrical \& Computer Engineering)
McMaster University

Hamilton, Ontario, Canada

TITLE:

Offset-Based Beamforming: A New Approach to Robust

Downlink Transmission

AUTHOR:

Mostafa Medra

B.Sc., M.Sc. (Electrical and Electronic Engineering)

Alexandria University,

Alexandria, Egypt

SUPERVISOR:

Timothy N. Davidson

NUMBER OF PAGES: xix, 164 
To my family and friends 


\section{Lay Abstract}

The increasing number of smart devices and Internet-based applications are driving the demand for higher data-rate wireless communication systems. One way to address that demand is to use multiple antennas at the base station to enable it to simultaneously serve multiple users instead of one. When such a base station is provided with the channel to each user, it can focus the energy of each data symbol towards the intended user while reducing the interference imposed on the other users. This is called beamforming. However, in practice the channel to each user is estimated and the beamformer design ought to include techniques that mitigate the impact of the resulting uncertainty. The goal of this thesis is to develop a suite of robust beamformer design techniques that do so, while also being of low-complexity, able to handle different power constraints, and being extendable to multi-cell systems in which multiple base stations may cooperate or coordinate in transmitting data to the users. 


\section{Abstract}

This thesis describes the design of low-complexity robust linear beamforming algorithms for multi-user downlink multiple-input single-output (MISO) communication systems. The goal of the algorithms is to provide the receivers with specified signalto-interference-and-noise ratios (SINRs) with high probability under certain power constraints. Unfortunately, the SINR outage constraint is intractable, and precise formulations of these problems are fundamentally hard to solve. The contribution of this thesis is a suite of algorithms that provide high-quality approximate solutions to a broad range of robust downlink beamforming problems, and do so at low computational cost. The unifying feature of these algorithms is that they are based, either explicitly or implicitly, on a transformation of each SINR outage constraint into a non-negativity constraint on a random variable, and the approximation of that non-negativity constraint by "offsets" on the mean of the distribution.

The first algorithm is developed for frequency division duplexing systems. Using a new extension of the S-Lemma, the channel uncertainty model is incorporated into the design problem using a zero-outage region approach. From that formulation, a new algorithm that is able to balance between the performance inside and outside the zero-outage region is developed. The resulting "offset maximization" algorithm has a low-complexity iterative closed-form solution that provides significant performance 
improvement, and can be extended to time division duplexing systems.

Analysis of the offset structure reveals a refined notion of the offset that incorporates information about each user's channel, and results in a convex semidefinite relaxation problem. When the channel uncertainty size is small, further approximations lead to an approximate iterative closed-form solution. When the beamforming directions are defined in advance, that algorithm provides near-optimal power loading.

Using subgradient methods, variants of the offset maximization algorithms that can accommodate per-antenna power constraints (PAPCs) are developed. Furthermore, the resulting offset-based power loading method can be combined with the maximum ratio transmission (MRT) or zero-forcing (ZF) directions, to provide robust algorithms that satisfy PAPCs with complexities low enough for massive MIMO applications.

Finally, the principles of the offset maximization algorithm are applied to multicell systems with the centralized cooperation, and with the centralized and decentralized architectures. The resulting algorithms provide significant performance improvement over those existing in the literature, and do so at substantially lower computational cost. 


\section{Acknowledgements}

I would like to thank my family, relatives and friends for their continuous support during my entire life. With them, I felt home in Canada.

I would like to thank my supervisory committee members Jun Chen, and Jian Kang Zhang for their insightful remarks and discussions during the committee meetings.

I would like to express my sincere appreciation to my supervisor, Timothy Davidson. Four years filled with accomplishments, work, joy and pleasure, is a great experience to live.

Finally, all praise and gratitude be to ALLAH. 


\title{
Abbreviations
}

\author{
BS base station \\ CSI channel state information \\ FDD frequency division duplexing \\ iid independent and identically distributed \\ KKT Karush-Kuhn-Tucker \\ LMI linear matrix inequality \\ MIMO multiple-input multiple-output \\ MISO multiple-input single-output \\ MRT maximum ratio transmission \\ MSE mean square error \\ OFDM orthogonal frequency division multiplexing \\ PAPC per-antenna power constraint \\ PAPR peak to average power ratio \\ QoS quality-of-service \\ RCI regularized channel inversion \\ RZF regularized zero-forcing \\ SB sphere bounding \\ SDR semidefinite relaxation
}


SINR signal-to-interference-and-noise ratio

SOC second order cone

TDD time division duplexing

ZF $\quad$ zero-forcing 


\section{Co-Authorship}

This thesis has been prepared in accordance with regulations for a "sandwich thesis" format. The thesis chapters two through five have been co-authored as follows:

\section{Chapter 2: Low-Complexity Robust MISO Downlink Precoder Design Under Imperfect CSI}

Authors: Mostafa Medra, Yongwei Huang, Wing-Kin Ma, and Timothy N. Davidson

Dr. Wing-Kin Ma helped with the formulation of the problem in (2.20), and Dr. Yongwei Huang provided the proof of the extension of the S-Lemma that was used in the solution of the problem in (2.20). That proof is provided in Appendix 2.A. Otherwise, the analysis and the simulations were carried by Mostafa Medra under the supervision of Dr. Timothy N. Davidson. The first draft of the paper was written by Mostafa Medra, under the supervision of Dr. Davidson, with modifications and revisions being made by all of the contributing authors. This chapter has been published in the IEEE Transactions on Signal Processing (Volume: 64, Issue: 12, Pages: 3237 - 3249, June 15, 2016). The copyright of the material in this chapter is held by the IEEE. The material is re-used with the permission of the IEEE, and this statement is included at the IEEE's request. 


\title{
Chapter 3: Offset-Based Beamforming: A New Approach to Robust Downlink Transmission
}

\author{
Authors: Mostafa Medra and Timothy N. Davidson
}

The refined notion of the offset maximization was conceived and developed by Mostafa Medra in the context of power loading (Medra and Davidson, 2015a). Dr. Yongwei Huang helped refine an initial extension of that notion to general beamforming (Medra and Davidson, 2016) which lead to the formulation of the problem in (3.11). Otherwise, the analysis, and the simulations were carried by Mostafa Medra under the supervision of Dr. Timothy N. Davidson. Mostafa Medra wrote the first draft of this paper, which has been revised in conjunction with Dr. Timothy N. Davidson, and is being finalized for submission to the IEEE Transactions on Signal Processing.

\section{Chapter 4: Low-Complexity Robust MISO Downlink Precoder Design With Per-Antenna Power Constraints} Authors: Mostafa Medra and Timothy N. Davidson

The analysis, and the simulations were carried by Mostafa Medra under the supervision of Dr. Timothy N. Davidson. Mostafa Medra wrote the first draft, which has been revised in conjunction with Dr. Timothy N. Davidson. This chapter has been submitted for possible publication in the IEEE Transactions on Signal Processing.

\section{Chapter 5: Low-Complexity Robust Multi-Cell MISO Downlink Precoder Design}

Authors: Mostafa Medra and Timothy N. Davidson

The analysis, and the simulations were carried by Mostafa Medra under the supervision of Dr. Timothy N. Davidson. Mostafa Medra wrote the first draft, which has 
been revised in conjunction with Dr. Timothy N. Davidson. This chapter has been published in the Proceedings of the 2016 IEEE International Conference on Acoustics, Speech and Signal Processing. The copyright of the material in this chapter is held by the IEEE. The material is re-used with the permission of the IEEE, and this statement is included at the IEEE's request. 


\section{Contents}

Lay Abstract $\quad$ iv

Abstract $\quad$ v

Acknowledgements vii

Abbreviations viii

Co-Authorship $\quad$ x

1 Introduction 1

1.1 Perfect CSI case beamforming . . . . . . . . . . . . . . . 2

1.2 Robust beamforming . . . . . . . . . . . . . . . 3

1.3 Robust power loading methods . . . . . . . . . . . . 5

1.4 Per-antenna power constraints . . . . . . . . . . . . 5

1.5 Multi-cell case . . . . . . . . . . . . . . . . . . . . 6

1.6 Thesis outline . . . . . . . . . . . . . . . . . . . . . . 7

2 Low-Complexity Robust MISO Downlink Precoder Design Under $\begin{array}{ll}\text { Imperfect CSI } & 11\end{array}$ 


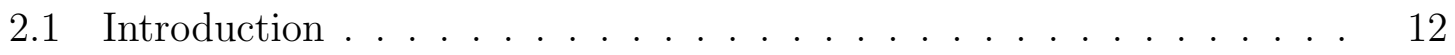

2.2 System Model . . . . . . . . . . . . . . . . . . . . . . . . . 15

2.2.1 Downlink precoding with perfect CSI _ . . . . . . . . 16

2.2.2 Principles of robust downlink precoding . . . . . . . . . . . 17

2.2 .3 Uncertainty models . . . . . . . . . . . . . . . . . . . . . . . . 19

2.3 Zero-outage region approach to the outage constrained problem . . . 21

2.4 Approaches to outage minimization problem . . . . . . . . 26

2.4.1 Zero-outage region maximization $\ldots \ldots \ldots \ldots$

2.4.2 Offset maximization for a given zero-outage region . . . . . . 28

2.4 .3 Offset maximization $\ldots \ldots \ldots \ldots$

2.4.4 Quasi-closed-form solution . . . . . . . . . . . . . . . . 32

2.4 .5 Computational cost . . . . . . . . . . . . . 34

2.4.6 Channel magnitude effect $\ldots \ldots \ldots \ldots$

2.5 Extensions to TDD case $\ldots \ldots \ldots \ldots \ldots \ldots \ldots$

2.6 Simulation studies $\ldots \ldots \ldots \ldots \ldots$

$2.6 .1 \quad$ FDD systems $\ldots \ldots \ldots \ldots \ldots$

2.6 .2 TDD systems $\ldots \ldots \ldots \ldots \ldots \ldots \ldots$

2.7 Conclusion . . . . . . . . . . . . . . . . . . . . . . . . . . . 43

2.A Appendix: Proof of Theorem $1 \ldots \ldots \ldots \ldots$

2.B Appendix: Bound derivation . . . . . . . . . . . . 50

2.C Appendix: Offset-maximization algorithm _... . . . . . 52

2.D Appendix: Rank one proof . . . . . . . . . . . . . . 53

3 Offset-Based Beamforming: A New Approach to Robust Downlink Transmission $\quad 55$ 


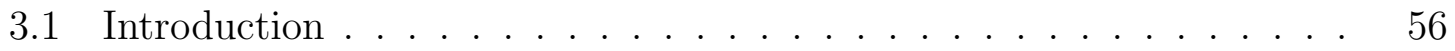

3.2 System model . . . . . . . . . . . . . . . . . . . . . . 60

3.3 Principles of Offset-based approach $\ldots \ldots \ldots \ldots$. . . . . . 62

3.4 Offset-based robust beamforming . . . . . . . . . . . . . . 64

3.4.1 Low-complexity precoding algorithm _ . . . . . . . . 66

3.4.2 Original constant-offset algorithm (Medra et al., 2016) _. . 71

3.4.3 Complexity analysis and further approximations . . . . . . 72

3.5 Offset-based robust power Loading . . . . . . . . . . . . . 74

3.5.1 Simplifying the SINR variance calculation $\ldots \ldots \ldots 76$

3.5 .2 User rescheduling . . . . . . . . . . . . . . . . . . 77

3.6 Simulation results . . . . . . . . . . . . . . . . . . . . . . . . . . . . 79

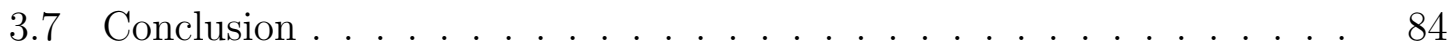

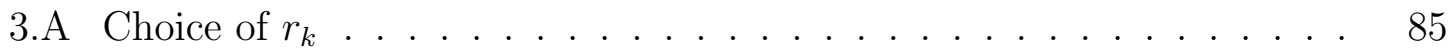

3.B Mean and variance derivations . . . . . . . . . . . . 85

4 Low-Complexity Robust MISO Downlink Precoder Design With $\begin{array}{lr}\text { Per-Antenna Power Constraints } & 87\end{array}$

4.1 Introduction . . . . . . . . . . . . . . . . . 88

4.2 System model and design approach $\ldots \ldots \ldots \ldots$

4.2 .1 Design approach . . . . . . . . . . . . . . . . . 93

4.2.2 Offset maximization beamforming directions . . . . . . . . 95

4.2 .3 Robust power loading . . . . . . . . . . . . . . . . 96

4.3 Offset maximization designs with PAPCs $\ldots \ldots \ldots \ldots$

4.3 .1 Dominant PAPCs . . . . . . . . . . . . . . . . . . . . . 101

4.3.2 Total and PAPCed algorithm . . . . . . . . . . 105 
4.3 .3 Algorithm acceleration . . . . . . . . . . . . . . . . 107

4.4 Conventional ZF beamforming with per-antenna power constraints . . 108

4.4.1 ZF beamforming with PAPCs only . . . . . . . . . . 111

4.4 .2 Generalized ZF beamforming . . . . . . . . . . . . . . . 113

4.5 Conventional MRT with per-antenna power constraints . . . . . . . 114

4.5.1 MRT with PAPCs . . . . . . . . . . . . . . . . 114

4.5.2 Generalized MRT . . . . . . . . . . . . . . . . . . . . . 119

4.6 Simulation results . . . . . . . . . . . . . . . . . . . . . . . . . . . . . 119

4.7 Conclusion . . . . . . . . . . . . . . . . . . . . . . . . . . . . 125

4.A Appendix: $\hat{\mathbf{Q}}$ update . . . . . . . . . . . . . . . . 126

5 Low-Complexity Robust Multi-Cell MISO Downlink Precoder De$\begin{array}{ll}\text { sign } & 128\end{array}$

5.1 Introduction . . . . . . . . . . . . . . . . . . . . . . 129

5.2 System Model . . . . . . . . . . . . . . . . . . . . . . . . 130

5.3 Offset-maximization for the single cell case . . . . . . . . . . . 132

5.4 Network MIMO offset-maximization . . . . . . . . . . . . . . . 135

5.4.1 Dominant total power constraint . . . . . . . . . . . 136

5.4.2 Dominant per-base station power constraints . . . . . . . . 137

5.5 Distributed algorithm based on virtual users . . . . . . . . . . . 139

5.6 Simulation results . . . . . . . . . . . . . . . . . . . . . . . . 142

5.7 Conclusion . . . . . . . . . . . . . . . . . . . . . . . . . . 144

6 Summary, conclusions and future work $\quad 145$

6.1 Summary . . . . . . . . . . . . . . . . . 145 
6.2 Conclusions . . . . . . . . . . . . . . . . . . . . . . . . . 147

6.3 Future work . . . . . . . . . . . . . . . . . . . . . 148 


\section{List of Figures}

2.1 Satisfaction probability=(1-outage probability) for the synthetic example involving an FDD system with 4 BS antennas, 3 users, and SINR targets $\gamma_{k}=9 \mathrm{~dB} \ldots \ldots \ldots \ldots \ldots \ldots \ldots \ldots \ldots \ldots$

2.2 Outage probability for an FDD system with 4 BS antennas, 3 users,

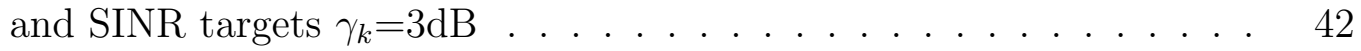

2.3 Outage probability for an FDD system with 4 BS antennas, 3 users, and SINR targets $\gamma_{k}=6 \mathrm{~dB} \ldots \ldots \ldots \ldots \ldots \ldots$

2.4 Outage probability for a TDD system with 4 BS antennas, 3 users, and SINR targets $\gamma_{k}=3 \mathrm{~dB} \ldots \ldots \ldots \ldots \ldots \ldots \ldots \ldots$

3.1 The average transmitted power against the outage probability for a system with 3 users, 4 BS antennas, $\gamma=6 \mathrm{~dB}$, and $\sigma_{e_{k}}=0.1 . \quad \ldots \quad 81$

3.2 The average transmitted power against the outage probability for a system with 3 users, 4 BS antennas, $\gamma=6 \mathrm{~dB}$, and $\sigma_{e_{k}}=0.05 \ldots \quad \ldots 2$

3.3 The average transmitted power against the outage probability for a system with 3 users, $4 \mathrm{BS}$ antennas, $\gamma=6 \mathrm{~dB}$, and $\sigma_{e_{k}}=0.1$. Here [15] referes to (Sohrabi and Davidson, 2016). . . . . . . . . . 83

3.4 The outage probability versus the number of antennas for a system with 6 users, $\gamma=6 \mathrm{~dB}$, and $\sigma_{e_{k}}=0.1 \ldots \ldots \ldots$ 
4.1 Outage probability for a 4 antenna BS serving 3 users with a total transmitted power of $P_{t}$. Here [18] refers to Medra and Davidson (2015a), and [23] refers to Yu and Lan (2007). . . . . . . . . . . . 122

4.2 Convergence behaviour for a 4 antenna BS serving 3 users with a total transmitted power of $P_{t}$. The violation probability measures the fraction of the 20,000 realizations for which at least one PAPC was violated by more then $10 \%$ at the given iteration of the algorithm.

4.3 Outage probability for a BS serving 3 users with a total transmitted power of $P_{t}=2$. Here [18] refers to Medra and Davidson (2015a), and [29] refers to Wiesel et al. (2008). . . . . . . . . . . . . . . . . . . 124

4.4 Outage probability for a BS serving 8 users with a total transmitted power of $P_{t}=1$. Here [18] refers to Medra and Davidson (2015a), and [24] refers to Feng and Jing (2016). . . . . . . . . . . . . . . . 125

5.1 Outage probability for 4 users, 4 antennas, $\gamma=3 \mathrm{~dB}, \sigma^{2}=-90 \mathrm{dBm}, \sigma_{h}=$ 0.2. Here [6] refers to Mirza et al. (2015), [7] refers to Qiu et al. (2010), and [10] refers to Pennanen et al. (2014) . . . . . . . . . . . . . . 144 


\section{Chapter 1}

\section{Introduction}

According to the annual visual network index reports that are released by Cisco, there is now quantitative evidence that the predicted rapid expansion in demand for wireless data has come to fruition and will continue (Cisco, 2016). This rapid expansion is mainly driven by the smart devices and the increasing number of applications. Accordingly, the recent report by Cisco and the forecast therein makes it plain that an incremental approach will not be enough to meet the network demands by 2020 (Cisco, 2016). One of the key technologies that next generation systems can use to meet the higher rate demands is to advance the use of multiple antennas. When the transmitter has many antennas instead of one, the transmitter can design complex-valued weights that can be applied to the symbols for multiple users and send those weighted symbols from the antennas in such a way that the signal adds constructively at the intended receiver, and destructively at other users. This is called beamforming. Beamforming can significantly increase the spectral efficiency at the receiver side without the need to change the users' equipment; a property that has made beamforming very appealing (e.g., Goldsmith et al., 2003; Gesbert et al., 
2007; Gershman et al., 2010). Although linear beamforming techniques do not enable a downlink system to operate at rates approaching the boundary of the capacity region, the complexity of the non-linear "dirty paper coding" technique (Costa, 1983) that is capacity achieving (Weingarten et al., 2006) is typically very high. Accordingly, the focus of this thesis will be on linear beamforming techniques. In this thesis, the focus will be on quality-of-service (QoS) type of problems. The bit error rate, and the capacity are among the most common QoS metrics, and both are functions of the signal-to-interference-and-noise ratio (SINR) (Wiesel et al., 2006). In the case of single-antenna receivers that are coherent, and the channels are memoryless, the linear QoS design problem can be reduced to optimizing the beamforming matrix to minimize the power required to satisfy predefined SINR constraints at the receivers (Schubert and Boche, 2007; Bengtsson and Ottersten, 2001; Rashid-Farrokhi et al., 1998a,b). As its name suggests, the SINR is the ratio of the power of the desired component of the received signal to the powers of the interference and noise components.

\section{$1.1 \quad$ Perfect CSI case beamforming}

When the base station (BS) has perfect knowledge of the channel state information (CSI), many conventional techniques can be used to control the signal and interference powers. The zero-forcing (ZF) method maximizes the received power of the desired signal while forcing the interference to be zero (Spencer et al., 2004). The regularized channel inversion (RCI) method allows for a little interference in order to achieve higher signal power (Peel et al., 2005). When the target is only to maximize the received signal power in noise-limited systems, maximum ratio transmission (MRT) 
can be used (Lo, 1999). Note that the ZF, RCI, and MRT precoding tackle the signal and interference powers separately, whereas the goal of the QoS design is to control the SINR itself. Fortunately, if the CSI at the BS is perfect, the problem of minimizing the transmitted power under prespecified SINR constraints can be solved, and an iterative closed-form solution can be derived (Bengtsson and Ottersten, 2001; Bjornson et al., 2014).

While conventional methods that assume perfect CSI are generally of low complexity, uncertainty in the CSI is inevitable. In frequency division duplexing (FDD) systems, the BS estimates the required CSI by transmitting training symbols to its users and then each user estimates the channel coefficients and sends a quantized version of the estimated channel vector back to the BS. In time division duplexing (TDD) systems, the BS designs the beamformers for the downlink phase based on the channel estimates that were obtained in the previous uplink slot, exploiting the channel reciprocity. In both settings, the BS has only estimates of the CSI and the performance of conventional beamforming techniques can be quite sensitive to the resulting uncertainty in the CSI, and consequently the BS's estimate of the SINRs at the receivers. Accordingly, the beamformer design ought to explicitly incorporate measures to mitigate the sensitivity to the estimation error (e.g., Kandukuri and Boyd, 2002).

\subsection{Robust beamforming}

There are several ways in which robustness to channel uncertainty can be explicitly incorporated into the beamforming design problem. The well-known "worst-case" approach involves finding a region around the estimated channel of each user so that 
the true channel lies in that region. Then the optimization problem is designed to provide a zero outage probability for any channel inside that region (e.g., Zheng et al., 2008; Shenouda and Davidson, 2007; Chang et al., 2011; Song et al., 2012; Shenouda and Davidson, 2008a; Huang et al., 2013; Vucic and Boche, 2009a; Ubaidulla and Chockalingam, 2008; Shenouda and Davidson, 2009). It is not always possible to find a region to contain the true channel (e.g., a Gaussian error is unbounded) which complicates the design problem. Alternatively, instead of that worst-case approach, the uncertainties can be modelled probabilistically and the requirement is then to minimize the total transmission power subject to the SINR constraints holding with a given probability. Accordingly, if a region can be defined in which the true channel lies with probability that is higher than $1-\delta_{k}$ in that region, and the beamformers are designed to provide a zero-outage probability in that region, then the resulting outage probability would be less than $\delta_{k}$ (e.g., Shenouda and Davidson, 2008b; Sohrabi and Davidson, 2016; He and Wu, 2013; Wang et al., 2014). In this case, the intractable SINR outage constraint is approximated by a zero-outage region constraint.

Both bounding methods use a "zero-outage" region approach, and that region is typically ellipsoidal. Using that region model, the worst-case or probabilistic approaches can be converted into convex optimization problems that can be efficiently solved. However, in most cases the optimization problem is complex and the computational complexity is high. In addition, the zero-outage region is by definition a conservative way to model uncertainty that provides no control to the performance outside that region. Accordingly, the resulting optimization problems are often infeasible, especially when the uncertainties are large, due to the conservative nature of the constraint. 


\subsection{Robust power loading methods}

The intricate nature of the SINR outage constraint has encouraged some authors try to decouple the problem into simpler problems. One way to do so is by choosing the beamforming directions in advance, and, then applying a robust power loading technique (e.g., Pascual-Iserte et al., 2006; Vucic and Boche, 2009b,a; Sohrabi and Davidson, 2016). Typically, the beamforming directions are chosen by applying a conventional design technique to the estimated channels. Vucic and Boche (2009b) suggested an iterative algorithm to minimize the mean square error (MSE) between the transmitted and received signal. When the uncertainty is additive and Gaussian, the optimal power loading in terms of the minimum power required by each user to achieve a certain outage probability can be obtained (Sohrabi and Davidson, 2016). However, the computational cost of that optimal algorithm is significant.

\subsection{Per-antenna power constraints}

In practical systems, each antenna at the transmitter has its own power amplifier which means that per-antenna power constraints (PAPCs) ought be added to the optimization problem. When the CSI is perfectly known at the BS, algorithms for incorporating the PAPCs into the conventional beamforming designs that separately control the signal and interference powers have been developed. Wiesel et al. (2008) designed beamformers that maximize the received signal power and cause no interference in addition to satisfying the PAPCs. This can be viewed as PAPCed-ZF beamforming. Since the ZF solution has a structure where the beamformer of each user should lie in the null space of other users' channels, Wiesel et al. (2008) were 
able to formulate the beamformer design problem as a second order cone (SOC) program that can be efficiently solved. Feng and Jing (2016) assumed nominal MRT beamforming and equal power loading, then scaled the beamforming vectors so that the PAPCs are met. Then they showed that the performance gap to MRT without PAPCs is negligible when the number of antennas is large. Note that the optimization problem of minimizing the total transmitted power under PAPCs to achieve certain SINR targets can be formulated as an optimization problem, and an iterative closed-form solution can be derived using subgradient methods (Yu and Lan, 2007).

One might think that the PAPCs can be directly added to the zero-outage based problems to provide robustness. While it is true that when the underlying optimization problem is convex, adding the PAPCs, which are convex constraints, will keep the problem convex, many of those optimization problems are based on semidefinite relaxation, and when the PAPCs are added there is no guarantee that the resulting solution will be close to being of rank one. Even if the resulting solution is rank-one, the computational complexity will definitely increase.

\subsection{Multi-cell case}

The single-cell beamforming schemes described above manage the intra-cell interference and assume that the interference from the other cells is managed independently, perhaps through a frequency allocation scheme that avoids reuse in neighbouring cells. However, there are opportunities to improve the performance of the network as a whole by allowing denser frequency reuse and jointly managing both the intra-cell and inter-cell interferences by jointly designing the beamformers that are to be used at each BS. The topology of the multi-cell scheme may be cooperative, in which multiple 
BSs work together to transmit the same information to each user, or coordinated, in which case each user is served by a single BS. When the BSs are coordinating, the beamformer design can be either centralized or distributed. Qiu et al. (2010) assumed a perfect CSI case, and suggested a bisection distributed algorithm for the maximum SINR target that can be achieved under a certain total power constraint. Pennanen et al. (2014) extended the algorithms that mitigate the uncertainty in the channel using a zero-outage region approach to accommodate for inter-cell interference. The centralized algorithm is then transformed into a distributed algorithm using dual decomposition and alternating direction method of multipliers-based approach. While this method provides robustness, the main draw back is the substantial computational cost and the conservative nature of the zero-outage region idea.

\subsection{Thesis outline}

The goal of this thesis is to suggest a new approach for robust downlink beamforming design, an approach that can provide algorithms with excellent performance and low computational cost. The design of beamforming vectors has many facets, including finding the beamforming directions to be used, and the power allocation for each user. Other facets include the addition of specific power constraints as the total power constraint or PAPCs, and the management of the inter-cell interference, as well as intra-cell interference, in different network architectures. Those design aspects should be addressed using algorithms that have low computational cost, so that they can be applied in high-rate systems. This thesis addresses the above mentioned aspects using a new offset-based approach. The proposed approach, in addition to addressing those aspects, also provides interesting power control and user admission features. 
This thesis is prepared in "sandwich" format, containing four self-contained chapters. Each chapter includes its own abstract, introduction, body, conclusion, and possibly appendices. For convenience, the references for each chapter are collected in a single bibliography at the end of the thesis.

Chapter 2 presents the problem of robust beamforming design in FDD systems using a zero-outage region approach. The design formulation takes into account the structure of the uncertainty that arises from downlink training and quantized feedback. By using an extension of the S-Lemma and a semidefinite relaxation, it is shown that the design problem can be formulated as a convex optimization problem. That convex optimization problem can be solved using interior point methods such as those available using the CVX tool in MatLaB (Grant et al., 2008). An analysis of the problem structure reveals that the performance outside the zero-outage region can be enhanced on the expense of a little performance degradation inside that region. In doing so, the resulting algorithm showed a better performance and has a structure that enabled the development of an iterative closed-form solution. The resulting "offset maximization" algorithm shares the same beamforming directions as the perfect CSI case (Bengtsson and Ottersten, 2001), but with different power loading. The results for TDD systems lead to similar conclusions.

Chapter 3 begins with the observation that despite being derived as the result of an approximation of linear matrix inequality (LMI) constraints, the offset maximization algorithm implicitly involves an approximation of the SINR outage constraint. Further investigation of this new approximation leads to an improved approximation of that outage constraint. Applying this new offset approximation, and a semidefinite 
relaxation, the beamforming design problem can be formulated as a convex optimization problem that contains SOC constraints and semidefinite constraints. When the uncertainty size is small, a further approximation allows for the development of an iterative closed-form solution. The performance gap between those approximations and the original formulation is shown to be small. The new offset approximation also provides a power loading technique when the beamforming directions are defined in advance. The resulting power loading algorithm performs quite well compared to the optimal power loading algorithm for additive Gaussian uncertainties (Sohrabi and Davidson, 2016). The proposed power loading provides an explicit relationship between the robustness required for each user and the power consumed by that user, which allows for simple, yet effective, power control techniques. The explicit relationship allows the transmitted power to be reduced when the channel is good, and can detect users with very weak or closely aligned channels. Such users typically consume most of the power resources, and may still be in outage. When that happens, the power can be allocated to other users, and the weak users can be rescheduled in other time or frequency slots.

Chapter 4 presents the application of PAPCs in the offset maximization framework. The PAPCed offset maximization algorithm makes use of insights from the subgradient methods developed by Yu and Lan (2007) for the perfect CSI case to provide a solution that satisfies the PAPCs. The near-optimal power loading can be combined into the design framework to provide robustness against channel uncertainties resulting in significant performance gains. The PAPCed offset maximization frame work can be extended to work with conventional beamforming directions as well. When the ZF and MRT beamforming directions are combined with the suggested robust 
power loading algorithms, the subgradient methods provide lower-computational cost algorithms that satisfy the PAPCs, while having reduced outage probability. When the number of antennas is large, the MRT-based PAPCed algorithm can be efficiently computed using operations that grow only linearly in the number of antennas.

Chapter 5 presents the application of the offset maximization family of algorithms to the multi-cell case. The offset maximization framework extends naturally to the cooperative case, and is shown to maintain its performance gains and the low-complexity design. In the coordinated distributed case, the offset maximization can be adapted to balance between providing better performance inside the cell, and decreasing the interference to other cells using very limited feedback.

Chapter 6 presents the summary and conclusions of the thesis, and suggests directions for future work. 


\title{
Chapter 2
}

\section{Low-Complexity Robust MISO Downlink Precoder Design Under Imperfect CSI}

\begin{abstract}
We consider the design of the linear precoder for a multiple-input single-output (MISO) downlink system with quality-of-service (QoS) constraints. The broad goal is to develop low-complexity techniques that mitigate the impact of the uncertainty in the transmitter's channel state information (CSI) by incorporating probabilistic models for the uncertainty into the design. The proposed techniques are developed for systems based on limited feedback, and they can be easily adapted to systems that acquire CSI using estimation on the uplink and channel reciprocity. We consider the conventional problem of minimizing the transmitted power under probability of outage constraints for a target signal-to-interference-and-noise ratio (SINR), and the
\end{abstract}


related problem of minimizing the outage probability under a transmitted power constraint. By approximating the outage constraint by a zero-outage region, employing a semidefinite relaxation, and applying an extension of the S-Lemma that is derived herein, these problems are converted into convex and quasi-convex problems, respectively. Insights into the structure of the solution of those problems are then used to generate an alternate design formulation that provides greater robustness in the presence of significant uncertainties and has a quasi-closed-form solution. As illustrated by simulations, the proposed alternate design provides significantly better performance than the conventional designs that do not incorporate uncertainty models, and better performance than existing robust designs in the presence of large uncertainties, and does so at a computational cost that is close to that of the conventional designs.

\section{$2.1 \quad$ Introduction}

In the communication of inelastic data traffic from a base station (BS) to multiple receivers, an effective strategy for the design of the transmitter is to seek to minimize the transmission power required to enable reliable communication to each receiver at the chosen data rates. Given the complexity of implementing the optimal encoding structure for the downlink (Weingarten et al., 2006), such quality-of-service (QoS) design problems are typically formulated for linear transmitters (e.g., Bengtsson and Ottersten, 2001; Rashid-Farrokhi et al., 1998a). In scenarios in which the receivers have a single antenna and are coherent, and the channels are memoryless, the linear QoS design problem reduces to optimizing the BS's precoding matrix so as to minimize the power required to satisfy signal-to-interference-and-noise ratio (SINR) constraints at the receivers (Bengtsson and Ottersten, 2001; Rashid-Farrokhi et al., 
1998a; Schubert and Boche, 2007; Wiesel et al., 2006). In order to perform this design, the BS must be able to determine the SINRs at the receivers as a function of the precoder. However, doing so requires the BS to obtain accurate information on the state of the channels to the receivers; i.e., accurate channel state information (CSI). In frequency division duplexing (FDD) systems, the BS obtains the required CSI by sending training symbols to the users and having each user estimate the channel coefficients and send a quantized version of the estimated channel vector back to the BS. In time division duplexing (TDD) systems, the uplink and the downlink operate in the same frequency band but in different time slots, and the BS designs its downlink precoder based on the channel estimate that was obtained in the previous uplink slot. In both settings, the BS has only estimates of the CSI, and hence can only estimate the SINR at each receiver. As a result, the precoder design must be performed in the presence of uncertainty in these estimates (e.g., Kandukuri and Boyd, 2002).

One approach to dealing with the uncertainty in the BS's estimates of the CSI is to develop a model for the uncertainty and incorporate that model into the design problem. One way to do that is to model the actual channel as lying in a compact set around the channel estimate at the BS, and to design the precoder so that the transmission power is minimized subject to the SINR constraints holding for all channels in the set (e.g., Zheng et al., 2008; Shenouda and Davidson, 2007; Chang et al., 2011; Song et al., 2012; Shenouda and Davidson, 2008a; Huang et al., 2013; Vucic and Boche, 2009a; Ubaidulla and Chockalingam, 2008; Shenouda and Davidson, 2009). (Analogous designs based on mean square error metrics are also available (Tseng and Gu, 2015; Zheng et al., 2009; Joham et al., 2012).) An alternative to that "worst-case" approach is to model the uncertainties probabilistically, and to seek to minimize the 
transmission power subject to the SINR constraints holding with a given probability; i.e., minimizing the transmission power subject to outage constraints (Shenouda and Davidson, 2008b; Sohrabi and Davidson, 2016; He and Wu, 2013; Wang et al., 2014). We will first develop techniques that are directly applicable to that problem. Then we will focus on the related problem of minimizing the outage probability subject to a constraint on the transmission power.

Many of the existing design techniques for probabilistic uncertainty models are based on conservative convex restrictions of the design problem that replace the chance constraint that enforces the specified outage condition by a deterministic constraint that specifies a set of channels over which zero outage is to be guaranteed (Shenouda and Davidson, 2008b; Wang et al., 2014; Pascual-Iserte et al., 2006). Typically, the set is contiguous and hence this generates a continuum of constraints, but doing so enables the application of existing techniques for the worst-case problem. Unfortunately, these techniques are inherently conservative and their performance degrades significantly in the presence of larger uncertainties or aggressive SINR targets. Furthermore, although the resulting design problems are convex, they typically involve linear matrix inequalities (LMIs), and the computational cost of solving them can be quite substantial. Finally, most of the existing approaches to this "zero-outage" conversion of outage-based problems have been developed for cases in which the uncertainty can be modelled as being additive and Gaussian. In TDD systems that exploit reciprocity, the Gaussian assumption is often quite reasonable (e.g., Sohrabi and Davidson, 2016), but in FDD systems that employ structured vector quantization schemes to feed CSI back to the BS, the Gaussian model is not appropriate.

In this paper we develop precoder design techniques that address these issues. 
We first develop a "zero-outage region" approach to approximating the problem of minimizing the transmission power required to achieve specified outage constraints for FDD schemes with structured vector quantization. That approach is enabled by the derivation, herein, of a variant of the S-Lemma for the complex-valued case. That technical result is also used to tackle the problem of outage minimization subject to a power constraint. Insight into the LMIs that arise in that problem enables the development of an alternate design approach. That approach generates quasi-closedform expressions for precoders that provide improved performance outside the zerooutage region. Our numerical experiments confirm the theoretical insights, and the resulting performance advantages can be quite significant in the presence of sizable uncertainties and aggressive SINR targets. The proposed designs extend naturally to the case of Gaussian uncertainties and similar performance advantages are achieved in the TDD case.

\subsection{System Model}

We consider a $K$-user unicast MISO downlink in which a BS equipped with $N_{t}$ antennas sends independent messages to $K$ single antenna users. The BS employs linear beamforming to construct the transmitted signal at each channel use, $\mathbf{x}=\sum_{k=1}^{K} \mathbf{w}_{k} s_{k}$, where $s_{k}$ is the normalized symbol intended for user $k$, and $\mathbf{w}_{k} \in \mathbb{C}^{N_{t}}$ is the associated beamformer. The signal received by the $k^{\text {th }}$ user is

$$
y_{k}=\mathbf{h}_{k}^{H} \mathbf{w}_{k} s_{k}+\sum_{j \neq k} \mathbf{h}_{k}^{H} \mathbf{w}_{j} s_{j}+n_{k}
$$


where $\mathbf{h}_{k}^{H} \in \mathbb{C}^{N_{t}}$ denotes the channel between the BS and receiver $k$, and $n_{k} \in \mathbb{C}$ represents the additive zero-mean circular complex Gaussian noise at that user.

\subsubsection{Downlink precoding with perfect CSI}

We will consider systems in which each user performs single-user detection, with the interference from other users being treated as noise. If receiver $k$ has obtained $\mathbf{h}_{k}^{H} \mathbf{w}_{k}$, say through a dedicated training step (Caire et al., 2010), then it can perform coherent detection. If, in addition, the noise and the interference terms in (2.1) are uncorrelated in time and their sum is approximately Gaussian, then the key performance metric of the link of user $k$ is the SINR,

$$
\operatorname{SINR}_{k}=\frac{\left|\mathbf{h}_{k}^{H} \mathbf{w}_{k}\right|^{2}}{\sum_{j \neq i}\left|\mathbf{h}_{k}^{H} \mathbf{w}_{j}\right|^{2}+\sigma_{k}^{2}},
$$

where $\sigma_{k}^{2}$ is the noise variance. In the case that the channel vectors and noise variances are known perfectly at the BS, the BS can design the beamformers to achieve a variety of goals (e.g., Gershman et al., 2010; Bjornson et al., 2014). One simple example is zero-forcing (ZF) beamforming (Spencer et al., 2004), in which each $\mathbf{w}_{k}$ is chosen such that $\mathbf{h}_{j}^{H} \mathbf{w}_{k}=0, \forall j \neq k$ and hence the interference is eliminated. A related beamforming technique is regularized channel inversion (RCI) (Peel et al., 2005). In this paper we will focus on beamforming techniques that provide a predefined level of QoS to each user (e.g., Bengtsson and Ottersten, 2001; Rashid-Farrokhi et al., 1998a; Schubert and Boche, 2007). In particular, we consider the problem in which the transmitter seeks to find a set of beamformers that minimizes the transmission power required to achieve certain SINR targets for each user. If we let $\gamma_{k}$ denote the 
target SINR for the $k^{\text {th }}$ user, that problem can be written as:

$$
\begin{aligned}
\min _{\left\{\mathbf{w}_{k}\right\}_{k=1}^{K}} & \sum_{k}\left\|\mathbf{w}_{k}\right\|^{2} \\
\text { subject to } & \mathrm{SINR}_{k} \geq \gamma_{k}, \quad k=1,2, \ldots, K,
\end{aligned}
$$

where $\|\cdot\|$ denotes the Euclidean norm. The formulation in (2.3) is not convex, but there are several efficient algorithms for finding globally optimum solutions (e.g., Bengtsson and Ottersten, 2001; Wiesel et al., 2006).

\subsubsection{Principles of robust downlink precoding}

In practice, the BS only has an estimate, $\hat{\mathbf{h}}_{k}$, of $\mathbf{h}_{k}$. Although a mismatched design in which the estimates $\hat{\mathbf{h}}_{k}$ are substituted for $\mathbf{h}_{k}$ in (2.3) could be attempted, the sensitivity of the SINR to the channel estimates suggests that it may be more effective to develop a model for the uncertainty and incorporate that model into the design. One way to do so is to postulate a conditional distribution $p_{k}\left(\mathbf{h}_{k} \mid \hat{\mathbf{h}}_{k}\right)$, select an outage probability $\delta_{k}$, then seek to minimize the transmission power subject to the $k^{\text {th }}$ SINR constraint holding with probability greater than or equal to $1-\delta_{k}$, or show that those specifications cannot be achieved; i.e., solve

$$
\begin{aligned}
\min _{\mathbf{w}_{k}} & \sum_{k}\left\|\mathbf{w}_{k}\right\|^{2} \\
\text { subject to } & \operatorname{Prob}\left(\operatorname{SINR}_{k} \geq \gamma_{k}\right) \geq 1-\delta_{k} .
\end{aligned}
$$

In (2.4) and the rest of the paper, we leave it implicit that the constraints hold for all $k \in\{1,2, \ldots, K\}$ and that the optimization is performed over the design variables associated with all users. We will also consider the related problem of minimizing the 
outage probability subject to a power constraint $P$; i.e.,

$$
\begin{array}{ll}
\min _{\mathbf{w}_{k}, \delta_{k}} \max _{k} & \delta_{k} \\
\text { subject to } & \operatorname{Prob}\left(\operatorname{SINR}_{k} \geq \gamma_{k}\right) \geq 1-\delta_{k}, \\
& \sum_{k}\left\|\mathbf{w}_{k}\right\|^{2} \leq P .
\end{array}
$$

In general, problems (2.4) and (2.5) are difficult to solve even for simple distributions. This is mainly due to the fact that even when deterministic expressions for the probabilistic constraints can be obtained, they are typically non-convex in the design parameters; see, (e.g., Sohrabi and Davidson, 2016).

One strategy for finding good solutions to the problem in (2.4) is to find a region $\mathcal{R}_{k}$ in which the channel $\mathbf{h}_{k}$ will lie with a probability of at least $1-\delta_{k}$ and ensure zero-outage in this region (e.g., Shenouda and Davidson, 2008b; Wang et al., 2014; Pascual-Iserte et al., 2006). That is, we design $\mathbf{w}_{k}$ so that

$$
\begin{aligned}
\min _{\mathbf{w}_{k}} & \sum_{k}\left\|\mathbf{w}_{k}\right\|^{2} \\
\text { subject to } & \operatorname{SINR}_{k} \geq \gamma_{k} \quad \forall \mathbf{h}_{k} \in \mathcal{R}_{k} .
\end{aligned}
$$

In other words, we approximate the chance constraint in (2.4b) by the deterministic, but semi-infinite, constraint in (2.6b). The constraint in (2.6b) is semi-infinite due to the continuum of members of $\mathcal{R}_{k}$. We note that, if $\mathcal{R}_{k}$ contains all the possible values that $\mathbf{h}_{k}$ can take on, then the problem in (2.6) is a typical worst-case problem. In certain cases, and in particular when $\mathcal{R}_{k}$ is a sphere centered on $\hat{\mathbf{h}}_{k}$, the worstcase problem in (2.6) can be conservatively bounded and solutions can be efficiently 
found (e.g., Zheng et al., 2008; Shenouda and Davidson, 2007). Although this method can be quite effective when the uncertainties are small, it is, by its very nature, conservative, and hence there may be instances in which (2.6) has no solution (i.e., (2.6) is infeasible) even though there are solutions to (2.4).

The concept of the zero-outage region can also be applied to the problem in (2.5). Rather than designing $\mathbf{w}_{k}$ to minimize the maximum outage, we can seek to maximize the volume of the zero-outage region; i.e.,

$$
\begin{array}{ll}
\underset{\mathbf{w}_{k}, \mathcal{R}_{k}}{\max } \min _{k} & \operatorname{vol}\left(\mathcal{R}_{k}\right) \\
\text { subject to } & \operatorname{SINR}_{k} \geq \gamma_{k}, \quad \forall \mathbf{h}_{k} \in \mathcal{R}_{k} \\
& \sum_{k}\left\|\mathbf{w}_{k}\right\|^{2} \leq P .
\end{array}
$$

A weakness of this formulation is that it simply attempts to maximize the size of the zero-outage region without regard for the behaviour outside this region. One of the main contributions of this paper will be to identify alternative strategies that enable the designer to balance the performance obtained when the uncertainties are small with the performance when the uncertainties are larger.

\subsubsection{Uncertainty models}

The specific techniques that we will develop in this paper are for FDD and TDD systems with quasi-static channels. We start with the case of an FDD system with structured vector quantization (Love et al., 2003). In these systems the receiver estimates the channel based on training signals sent by the BS and separately quantizes the gain and "direction" of the channel. More specifically, if we let $\tilde{\mathbf{h}}_{k}$ denote the receiver's estimate, the receiver quantizes $\sqrt{\alpha_{k}}=\left\|\tilde{\mathbf{h}}_{k}\right\|$ using a scalar quantizer and 
quantizes $\tilde{\mathbf{h}}_{n_{k}}=\tilde{\mathbf{h}}_{k} /\left\|\tilde{\mathbf{h}}_{k}\right\|$ using memoryless vector quantization over a Grassmannian codebook (Love et al., 2003); i.e., if $\mathcal{C}=\left\{\mathbf{v}_{1}, \mathbf{v}_{2}, \ldots, \mathbf{v}_{M}\right\}$ denotes a Grassmannian codebook of $M$ elements in $\mathbb{C}^{N_{t}}$, the codebook element that characterizes the direction of the channel is represented by $\mathbf{h}_{q_{k}}=\arg \min _{\mathbf{v} \in \mathcal{C}} 1-\left|\tilde{\mathbf{h}}_{n_{k}}^{H} \mathbf{v}\right|^{2}$. If we assume that (i) $\tilde{\mathbf{h}}_{k}$ is estimated accurately, i.e. perfect channel state information at the receiver (CSIR); (ii) $\alpha_{k}$ is quantized at a high resolution; and (iii) there are no errors in the feedback path, then the transmitter's estimate of the channel, $\sqrt{\alpha_{k}} \mathbf{h}_{q_{k}}$, is related to the actual channel by:

$$
\mathbf{h}_{k}=\sqrt{\alpha_{k}}\left(\mathbf{h}_{q_{k}}+\mathbf{e}_{k}\right)
$$

where the statistics of the error $\mathbf{e}_{k}$ are dependent on the codebook and the statistics of the channel. Given the intricate nature of the statistics of $\mathbf{e}_{k}$, even in the context of randomized codebooks (Jindal, 2006), our initial development will be based on a model in which $\mathbf{e}_{k}$ lies in a region $\mathcal{E}_{k}$ that is a spherical cap on the Grassmannian manifold of radius $\varepsilon$, centered at $\mathbf{h}_{q_{k}}$; i.e.,

$$
\mathcal{E}_{k}=\left\{\mathbf{e}_{k} \in \mathbb{C}^{N_{t}}:\left\|\mathbf{e}_{k}\right\| \leq \varepsilon,\left\|\mathbf{h}_{q_{k}}+\mathbf{e}_{k}\right\|=1\right\} .
$$

In practice, it is likely that each user in the cell will use a rotated version of the same codebook (Ding et al., 2007), and hence a common value for $\varepsilon$ is appropriate. However, the techniques below extend naturally to scenarios in which a different $\varepsilon$ for each user would be appropriate. As we will see in Sections 2.3 and 2.4, respectively, we can incorporate this uncertainty model into both problems (2.6) and (2.7).

In the TDD case, the BS estimates the channel during the uplink training phase. Assuming that (i) the channel is quasi-static; (ii) that appropriate compensation of 
the RF chain is employed (e.g., Kaltenberger et al., 2010), the transmitter's estimate $\mathbf{h}_{e_{k}}$ can be related to the channel $\mathbf{h}_{k}$ as

$$
\mathbf{h}_{k}=\mathbf{h}_{e_{k}}+\mathbf{e}_{k},
$$

where $\mathbf{e}_{k}$ is a zero mean complex Gaussian random variable, whose covariance is determined by the statistics of the channel and the training sequence (Poor, 1994). This uncertainty model will be considered in Section 2.5.

\subsection{Zero-outage region approach to the outage con- strained problem}

Let us first consider the problem of minimizing the transmission power subject to a zero-outage constraint (cf. (2.6)) for an FDD system. By defining $\mathbf{W}_{k} \in \mathbb{C}^{N_{t} \times N_{t}}$, where $\mathbf{W}_{k}=\mathbf{w}_{k} \mathbf{w}_{k}^{H}$ and applying the uncertainty model in (2.8), the SINR expression for user $k$ can be written as

$$
\operatorname{SINR}_{k}\left(\mathbf{e}_{k}\right)=\frac{\alpha_{k}\left(\mathbf{h}_{q_{k}}+\mathbf{e}_{k}\right)^{H} \mathbf{W}_{k}\left(\mathbf{h}_{q_{k}}+\mathbf{e}_{k}\right)}{\alpha_{k}\left(\mathbf{h}_{q_{k}}+\mathbf{e}_{k}\right)^{H}\left(\sum_{j \neq k} \mathbf{W}_{j}\right)\left(\mathbf{h}_{q_{k}}+\mathbf{e}_{k}\right)+\sigma_{k}^{2}} .
$$


Therefore, for a given uncertainty size $\varepsilon$, the worst-case problem in (2.6) can be written as

$$
\begin{array}{ll}
\min _{\mathbf{W}_{k}} & \sum_{k=1}^{K} \operatorname{tr}\left(\mathbf{W}_{k}\right) \\
\text { subject to } & \operatorname{SINR}_{k}\left(\mathbf{e}_{k}\right) \geq \gamma_{k}, \quad \forall \mathbf{e}_{k} \in \mathcal{E}_{k}, \\
& \mathbf{W}_{k} \succeq \mathbf{0}, \operatorname{rank}\left(\mathbf{W}_{k}\right)=1,
\end{array}
$$

where $\mathcal{E}_{k}$ was defined in (2.9), $\operatorname{tr}(\cdot)$ denotes the trace of a matrix, and $\mathbf{A} \succeq \mathbf{B}$ denotes that the matrices $\mathbf{A}$ and $\mathbf{B}$ are Hermitian symmetric and that $\mathbf{A}-\mathbf{B}$ is positive semidefinite.

The problem in (2.12) is difficult to solve for two reasons. First, the rank constraint is non-convex. Second, the SINR constraints are non-convex and there is an infinite number of them. We will address the first difficulty by removing the rank constraint, which results in a semidefinite relaxation (SDR) of the problem (Bengtsson and Ottersten, 2001; Gershman et al., 2010; Luo et al., 2010). The extraction of "good" beamformers $\mathbf{w}_{k}$ from that relaxation will be discussed below.

To address the second difficulty we rewrite the SINR constraint in (2.12b) as

$$
\left(\mathbf{h}_{q_{k}}+\mathbf{e}_{k}\right)^{H} \mathbf{Q}_{k}\left(\mathbf{h}_{q_{k}}+\mathbf{e}_{k}\right)-\sigma_{k}^{2} / \alpha_{k} \geq 0,
$$

where $\mathbf{Q}_{k} \in \mathbb{C}^{N_{t} \times N_{t}}$ is defined as

$$
\mathbf{Q}_{k}=\mathbf{W}_{k} / \gamma_{k}-\sum_{j \neq k} \mathbf{W}_{j}
$$


and we rewrite $\mathcal{E}_{k}$ in $(2.9)$ as

$$
\mathcal{E}_{k}=\left\{\mathbf{e}_{k} \in \mathbb{C}^{N_{t}}:\left\|\mathbf{e}_{k}\right\|^{2}-\varepsilon^{2} \leq 0,\left\|\mathbf{e}_{k}\right\|^{2}+2 \operatorname{Re}\left\{\mathbf{e}_{k}^{H} \mathbf{h}_{q_{k}}\right\}=0\right\} .
$$

Here, $\operatorname{Re}\{\cdot\}$ denotes the real part of the argument. By doing so, the constraint in (2.12b) can be reformulated as requiring (2.13) to hold for all $\mathbf{e}_{k}$ satisfying both the quadratic inequality and the quadratic equality in (2.15). That reformulation enables the application of the following theorem that precisely characterizes this semi-infinite constraint in a finite manner. To state the theorem concisely, we let $\mathbb{H}^{n \times n}$ denote the set of Hermitian symmetric matrices of size $n \times n$.

Theorem 1 Let $f_{i}(\mathbf{x})=\mathbf{x}^{H} \mathbf{A}_{i} \mathbf{x}+2 \operatorname{Re}\left\{\mathbf{x}^{H} \mathbf{b}_{i}\right\}+c_{i}, i=0,1,2$, where $\mathbf{x} \in \mathbb{C}^{n}$ and $\mathbf{A}_{i} \in \mathbb{H}^{n \times n}, \mathbf{b}_{i} \in \mathbb{C}^{n}, c_{i} \in \mathbb{R}, i=0,1,2$. Define

$$
\mathbf{F}_{i}=\left(\begin{array}{cc}
\mathbf{A}_{i} & \mathbf{b}_{i} \\
\mathbf{b}_{i}^{H} & c_{i}
\end{array}\right), \quad i=0,1,2 .
$$

Suppose that $\mathbf{F}_{2}$ is indefinite, and that there exists a vector $\mathbf{x}_{0} \in \mathbb{C}^{n}$ such that $f_{1}\left(\mathbf{x}_{0}\right)<$ 0 and $f_{2}\left(\mathbf{x}_{0}\right)=0$. Then the following two statements are equivalent:

1. $f_{0}(\mathbf{x}) \geq 0$ for all $\mathbf{x}$ satisfying $f_{1}(\mathbf{x}) \leq 0$ and $f_{2}(\mathbf{x})=0$.

2. There exists $\lambda \geq 0, \mu \in \mathbb{R}$ such that

$$
\mathbf{F}_{0}+\lambda \mathbf{F}_{1}+\mu \mathbf{F}_{2} \succeq \mathbf{0} .
$$

The above theorem can be viewed as an extension of the S-Lemma (Pólik and Terlaky, 2007) to the complex-valued case with one additional equality constraint. The proof is 
provided in Appendix 2.A. The proof leverages the fact that certain complex-valued semidefinite programs can be shown to have rank-one solutions (Huang and Zhang, 2007).

To apply Theorem 1 to the problem in hand, we associate the function $f_{0}$ with the expression on the left hand side of (2.13), the function $f_{1}$ with $\left\|\mathbf{e}_{k}\right\|^{2}-\varepsilon^{2}$ and the function $f_{2}$ with $\left\|\mathbf{e}_{k}\right\|^{2}+2 \operatorname{Re}\left\{\mathbf{e}_{k}^{H} \mathbf{h}_{q_{k}}\right\}$. Doing so enables us to transform the semiinfinite SINR constraint for each user in (2.12b) into a finite matrix inequality of the form

$$
\left(\begin{array}{cc}
\mathbf{Q}_{k} & \mathbf{Q}_{k} \mathbf{h}_{q_{k}} \\
\mathbf{h}_{q_{k}}^{H} \mathbf{Q}_{k} & \mathbf{h}_{q_{k}}^{H} \mathbf{Q}_{k} \mathbf{h}_{q_{k}}-\sigma_{k}^{2} / \alpha_{k}
\end{array}\right)+\lambda_{k}\left(\begin{array}{cc}
\mathbf{I} & \mathbf{0} \\
\mathbf{0} & -\varepsilon^{2}
\end{array}\right)+\mu_{k}\left(\begin{array}{cc}
\mathbf{I} & \mathbf{h}_{q_{k}} \\
\mathbf{h}_{q_{k}}^{H} & 0
\end{array}\right) \succeq \mathbf{0} .
$$

For ease of notation we will write (2.18) as $\mathbf{F}_{0 k}\left(\mathbf{Q}_{k}\right)+\lambda_{k} \mathbf{F}_{1}(\varepsilon)+\mu_{k} \mathbf{F}_{2 k} \succeq \mathbf{0}$, and we observe that $\mathbf{F}_{0 k}\left(\mathbf{Q}_{k}\right)$ and $\mathbf{F}_{1}(\varepsilon)$ are linear in their arguments, and that $\mathbf{Q}_{k}$ is linear in each $\mathbf{W}_{j}$.

Employing the above transformation, the relaxation of (2.12) obtained by removing the rank constraints on $\mathbf{W}_{k}$ can be written as

$$
\begin{array}{cl}
\min _{\mathbf{W}_{k}, \lambda_{k}, \mu_{k}} & \sum_{k=1}^{K} \operatorname{tr}\left(\mathbf{W}_{k}\right) \\
\text { subject to } & \mathbf{F}_{0 k}\left(\mathbf{Q}_{k}\right)+\lambda_{k} \mathbf{F}_{1}(\varepsilon)+\mu_{k} \mathbf{F}_{2 k} \succeq \mathbf{0}, \\
& \mathbf{W}_{k} \succeq \mathbf{0}, \quad \lambda_{k} \geq 0 .
\end{array}
$$

The problem in (2.19) has a linear objective, and constraints in the form of scalar linear inequalities and LMIs. Hence, it is a convex problem and can be efficiently solved using interior point methods (e.g., Sturm, 1999). Implementations of those 
methods can be conveniently accessed using the Matlab-based CVX interface (Grant et al., 2008). In the generic case, the remaining step is to use the optimal matrices $\left\{\mathbf{W}_{k, \mathrm{SDR}}^{\star}\right\}$ for the problem in (2.19) to generate feasible rank-one solutions to (2.12) with low objective values using randomization techniques (Wang et al., 2014; Luo et al., 2010). In Appendix 2.B we adapt derivations used in (Song et al., 2012) to show that for sufficiently small $\varepsilon$ there is guaranteed to be an optimal solution to (2.19) in which all $\mathbf{W}_{k, \mathrm{SDR}}^{\star}$ have rank one. Furthermore, consistent with the observations of others for related problems (e.g., Chang et al., 2011; Song et al., 2012; Wang et al., 2014), our experience with numerical experiments for larger values of $\varepsilon$ has shown that when the problem in (2.19) is feasible it almost always has a solution in which each $\mathbf{W}_{k, \text { SDR }}^{\star}$ has rank one.

To conclude this section we revisit the original goal, which was to develop an effective algorithm for the outage constrained problem in (2.4) in the case of channel "directions" feedback. The development was based on the zero-outage region approach in (2.6). As we will show in our numerical results in Section 2.6, the proposed approach is indeed effective when the distribution of the uncertainties is concentrated in a region in which $\mathbf{e}_{k}$ is relatively small. (Those observations are consistent with the observations in related works (e.g., Zheng et al., 2008; Shenouda and Davidson, 2007; Wang et al., 2014).) However, in the limited feedback systems that have currently been envisioned, the number of bits allocated to the feedback process is small (e.g., Love et al., 2008). Therefore, the quantization codebooks are quite small and, as a result, the probability of having sizeable uncertainties can be reasonably high. When the size of the zero-outage regions are increased to cover the bulk of uncertainties in envisioned systems, the conservatism in the zero-outage approach becomes apparent 
and the relaxed design problem in (2.19) is often infeasible. (That observation is also consistent with those in related works (e.g., Zheng et al., 2008; Shenouda and Davidson, 2007; Wang et al., 2014).) For that reason, we will now examine the application of the zero-outage region approach, and other ad-hoc approaches, to the outage minimization problem in (2.5).

\subsection{Approaches to outage minimization problem}

In this section we develop several approaches for generating good solutions to the outage minimization problem in (2.5) for FDD systems. We will begin with an approach that seeks to maximize a zero-outage region. Insights obtained from that approach will drive the development of an alternate approach that yields a quasi-closed-form solution.

\subsubsection{Zero-outage region maximization}

To apply the zero-outage region maximization approach in (2.7) to the case of FDD systems we choose the region $\mathcal{E}_{k}$ to contain all $\mathbf{e}_{k}$ satisfying (2.9), and we use the radius $\varepsilon$ as a measure of the volume. With those choices in place, the semidefinite relaxation of (2.7) in the case of FDD systems can be written as 


$$
\begin{array}{cl}
\underset{\mathbf{W}_{k}, \lambda_{k}, \mu_{k}, \varepsilon}{\max } & \varepsilon \\
\text { subject to } & \sum_{k=1}^{K} \operatorname{tr}\left(\mathbf{W}_{k}\right) \leq P, \\
& \mathbf{F}_{0 k}\left(\mathbf{Q}_{k}\right)+\lambda_{k} \mathbf{F}_{1}(\varepsilon)+\mu_{k} \mathbf{F}_{2 k} \succeq \mathbf{0}, \\
& \mathbf{W}_{k} \succeq \mathbf{0}, \quad \lambda_{k} \geq 0 .
\end{array}
$$

Since (2.20c) contains the bilinear term $\lambda_{k}\left(\begin{array}{cc}\mathbf{I} & \mathbf{0} \\ \mathbf{0} & -\varepsilon^{2}\end{array}\right)$, the problem in $(2.20)$ is not convex. However, when $\varepsilon$ is fixed the problem is convex in the other variables, and the feasible sets in (2.20) with increasing values of $\varepsilon$ are nested within each other. Therefore, an optimal solution to (2.20) can be efficiently found by performing a bisection search on $\varepsilon$ in which the problem solved at each step is the feasibility problem that arises when $\varepsilon$ is fixed in (2.20).

As alluded to earlier, a weakness in the zero-outage region maximization approach in (2.7), and hence a weakness in the formulation in (2.20), is that it attempts to maximize the zero-outage region without regard for the behaviour outside this region. In the case of Gaussian uncertainties, the size of the zero-outage region that can be achieved is relatively small (e.g., Shenouda and Davidson, 2008b; Wang et al., 2014). As we will see in Section 2.6, that is also true in the limited feedback case. In the next subsection we will develop an approximation of (2.20) that provides better performance for larger uncertainties. 


\subsubsection{Offset maximization for a given zero-outage region}

As stated in the original chance constraints in $(2.5 \mathrm{~b})$, what we are really seeking is a set of beamformers $\left\{\mathbf{w}_{k}\right\}$ that ensures that for each user $k, \operatorname{SINR}_{k} \geq \gamma_{k}$ with high probability. Using the notation in (2.13), we can express that constraint as

$$
\mathbf{h}_{q_{k}}^{H} \mathbf{Q}_{k} \mathbf{h}_{q_{k}}+2 \operatorname{Re}\left\{\mathbf{e}_{k}^{H} \mathbf{Q}_{k} \mathbf{h}_{q_{k}}\right\}+\mathbf{e}_{k}^{H} \mathbf{Q}_{k} \mathbf{e}_{k}-\sigma_{k}^{2} / \alpha_{k} \geq 0
$$

holding with high probability. To gain some insight into how this is achieved in (2.20), consider the "South-East" block of the LMI in (2.18). This ensures that any feasible point for (2.20) satisfies

$$
\mathbf{h}_{q_{k}}^{H} \mathbf{Q}_{k} \mathbf{h}_{q_{k}}-\sigma_{k}^{2} / \alpha_{k}-\lambda_{k} \varepsilon^{2} \geq 0 .
$$

A comparison between (2.21) and (2.22) shows that for a zero-outage region of a given size $\varepsilon$, the feasible points of (2.20) with larger values of $\lambda_{k}$ have larger values for $\mathbf{h}_{q_{k}}^{H} \mathbf{Q}_{k} \mathbf{h}_{q_{k}}-\sigma_{k}^{2} / \alpha_{k}$. Therefore, they have greater robustness to uncertainties of size larger than $\varepsilon$. That is true because the error $\mathbf{e}_{k}$ has to make $2 \operatorname{Re}\left\{\mathbf{e}_{k}^{H} \mathbf{Q}_{k} \mathbf{h}_{q_{k}}\right\}+\mathbf{e}_{k}^{H} \mathbf{Q}_{k} \mathbf{e}_{k}$ "more negative" in order for (2.21) not to be satisfied. We will now use that insight to develop an alternate ad-hoc algorithm that yields solutions with a smaller zero-outage region, but with greater robustness against larger uncertainties.

Let $\varepsilon^{\star}$ denote the optimal value of (2.20). Our goal now is to give up a little of the zero-outage region in order to obtain larger values for the offset, $\lambda_{k} \varepsilon^{2}$, and hence greater robustness to larger uncertainties. If we seek to maximize the minimum offset

for a given value of $\varepsilon$, denoted by $\varepsilon_{0}$, that satisfies $\varepsilon_{0}<\varepsilon^{\star}$, the semidefinite relaxation 
of the problem can be formulated as

$$
\begin{array}{rl}
\max _{\mathbf{W}_{k}, \lambda_{k}, \mu_{k}, t} & t \\
\text { subject to } & \sum_{k=1}^{K} \operatorname{tr}\left(\mathbf{W}_{k}\right) \leq P, \\
& \mathbf{F}_{0 k}\left(\mathbf{Q}_{k}\right)+\lambda_{k} \mathbf{F}_{1}\left(\varepsilon_{0}\right)+\mu_{k} \mathbf{F}_{2 k} \succeq \mathbf{0}, \\
& \mathbf{W}_{k} \succeq \mathbf{0}, \quad \lambda_{k} \geq t, \quad t \geq 0 .
\end{array}
$$

This problem is convex in all the design parameters and can be efficiently solved.

To assess the structure of solutions to this problem, we observe that given $\varepsilon_{0}<\varepsilon^{\star}$, if $\left\{\mu_{k}^{\star}\right\}$ and $\left\{\lambda_{k}^{\star}\right\}$ denote the optimal values for (2.23) and if $\mathbf{Q}_{k}^{\star}$ denotes the value of $\mathbf{Q}_{k}$ for the optimal $\left\{\mathbf{W}_{k}^{\star}\right\}$, then from (2.23c) we have

$$
\mathbf{F}_{0 k}\left(\mathbf{Q}_{k}^{\star}\right)+\lambda_{k}^{\star} \mathbf{F}_{1}\left(\varepsilon_{0}\right)+\mu_{k}^{\star} \mathbf{F}_{2 k} \succeq \mathbf{0} .
$$

To explore some of the scaling effects we observe that for any $a \geq 1$, we can rewrite the LMI in (2.24) as

$$
\mathbf{F}_{0 k}\left(\mathbf{Q}_{k}^{\star}\right)+a \lambda_{k}^{\star} \mathbf{F}_{1}\left(\varepsilon_{0} / \sqrt{a}\right)+\mu_{k}^{\star} \mathbf{F}_{2 k}-(a-1) \lambda_{k}^{\star}\left(\begin{array}{cc}
\mathbf{I} & \mathbf{0} \\
\mathbf{0} & 0
\end{array}\right) \succeq \mathbf{0} .
$$

Since $(a-1) \lambda_{k}^{\star}\left(\begin{array}{ll}\mathbf{I} & 0 \\ 0 & 0\end{array}\right)$ is positive semidefinite, this implies that if we reduce $\varepsilon_{0}$ by a factor $\sqrt{a}$, we can increase $\lambda_{k}$ by at least a factor $a$ while maintaining feasibility. This implies that if we solve (2.23) for a given $\varepsilon_{0}<\varepsilon^{\star}$, then the resulting offset, $\lambda_{k}^{\star} \varepsilon_{0}^{2}$, will be at least as large as the corresponding offset provided by the optimal solution to (2.20). In Section 2.6, we will provide an illustration of how the choice of $\varepsilon_{0}$ provides 
a trade-off between the size of the zero-outage region and the performance outside that region.

\subsubsection{Offset maximization}

Although the problem in (2.23) is convex, the $K$ LMIs in (2.23c) impose a significant computational burden on the solver; see Section 2.4.5. To examine opportunities to develop an approximation of (2.23) that may be amenable to a more efficient solution, we observe that the South-East block of (2.23c), which appears in (2.22), is the only block in which the term $\lambda_{k}$ appears with a negative multiplier. This suggests that this block, which is only a scalar, may be the dominant block in (2.23c). By using insights from the Schur Complement Theorem and the scaling arguments in the previous subsection, it can be shown that as $\varepsilon_{0}$ decreases, the South-East block of the LMI in (2.23c) does indeed become the dominant component of the constraint. In particular, the South-East block of the LMI does not depend on $\mu_{k}$, and as $\varepsilon_{0}$ is decreased, if the South-East block of the LMI is satisfied, it becomes increasingly likely that a value of $\mu_{k}$ can be found such that the LMI holds. (The details of that argument are implicit in Appendix 2.C) This observation suggests the following approximation of (2.23) in which each LMI is replaced by the scalar South-East block (cf. (2.22)). Defining $r_{k}=\lambda_{k} \varepsilon_{0}^{2}$, the approximation of (2.23) is

$$
\begin{array}{rl}
\max _{\mathbf{W}_{k}, r_{k}, t} & t \\
\text { subject to } & \sum_{k=1}^{K} \operatorname{tr}\left(\mathbf{W}_{k}\right) \leq P, \\
& \mathbf{h}_{q_{k}}^{H} \mathbf{Q}_{k} \mathbf{h}_{q_{k}}-\sigma_{k}^{2} / \alpha_{k}-r_{k} \geq 0, \\
& \mathbf{W}_{k} \succeq \mathbf{0}, \quad r_{k} \geq t, \quad t \geq 0 .
\end{array}
$$


In Appendix 2.C we show that this approximation is asymptotically tight in the sense that as $\varepsilon_{0} \rightarrow 0$ any optimal set $\left\{\mathbf{W}_{k}\right\}$ for (2.26) approaches an optimal set $\left\{\mathbf{W}_{k}\right\}$ for $(2.23)$.

Further simplification is also possible. Indeed, as we show in Appendix 2.D, there is an optimal solution of (2.26) in which all the $r_{k}$ 's are equal. As a result, the following simpler problem generates an optimal solution to (2.26):

$$
\begin{aligned}
\underset{\mathbf{W}_{k}, r}{\max } & r \\
\text { subject to } & \sum_{k=1}^{K} \operatorname{tr}\left(\mathbf{W}_{k}\right) \leq P, \\
& \mathbf{h}_{q_{k}}^{H} \mathbf{Q}_{k} \mathbf{h}_{q_{k}}-\sigma_{k}^{2} / \alpha_{k}-r \geq 0, \\
& \mathbf{W}_{k} \succeq \mathbf{0}, \quad r \geq 0 .
\end{aligned}
$$

In Appendix 2.D we use a minor variation of a result of Bengtsson and Ottersten (2001) to show that the problem in (2.27) has an optimal solution in which each $\mathbf{W}_{k}$ has rank one, and in Section 2.4.4 below we will show that a rank-one solution can be obtained in a quasi-closed form.

The SINR constraint in $(2.27 \mathrm{c})$ has an interesting interpretation. If we recall that the BS's estimate of $\mathbf{h}_{k}$ is $\hat{\mathbf{h}}_{k}=\sqrt{\alpha_{k}} \mathbf{h}_{q_{k}}$, the SINR condition can be rewritten as

$$
\frac{\hat{\mathbf{h}}_{k}^{H} \mathbf{W}_{k} \hat{\mathbf{h}}_{k}}{\hat{\mathbf{h}}_{k}^{H}\left(\sum_{j \neq k} \mathbf{W}_{j}\right) \hat{\mathbf{h}}_{k}+\sigma_{k}^{2}+\alpha_{k} r} \geq \gamma_{k} .
$$

That is, the problem in (2.27) obtains robustness to uncertainties in the CSI by seeking, with a particular affine scaling, the largest noise variances for which a mismatched design can satisfy the original SINR constraints. The expression in (2.28) also shows 
that if the problem in (2.27) is not feasible, then even in the perfect CSI case, there is no precoder of power less than or equal to $P$ that can satisfy the SINR requirements. More precisely, the perfect CSI problem in (2.3) with channels $\mathbf{h}_{i}=\hat{\mathbf{h}}_{k}$ has no feasible solution with that power constraint.

\subsubsection{Quasi-closed-form solution}

The expression in (2.28) not only provides an interesting interpretation of the problem in (2.27), it also facilitates the development of a robust precoder design problem that has a quasi-closed-form solution. In particular, if $r^{\star}$ denotes the optimal value of $r$ in (2.27), then since (2.27) has an optimal solution with rank one $\mathbf{W}_{k}$ 's, for any $r_{0} \leq r^{\star}$ we can directly obtain the beamformers that provide a feasible solution to (2.27) by solving

$$
\begin{array}{ll}
\min _{\mathbf{w}_{k}} & \sum_{k} \mathbf{w}_{k}^{H} \mathbf{w}_{k} \\
\text { s.t. } & \frac{\alpha_{k} \mathbf{h}_{q_{k}}^{H} \mathbf{w}_{k} \mathbf{w}_{k}^{H} \mathbf{h}_{q_{k}}}{\alpha_{k} \mathbf{h}_{q_{k}}^{H} \sum_{j \neq k} \mathbf{w}_{j} \mathbf{w}_{j}^{H} \mathbf{h}_{q_{k}}+\sigma_{k}^{2}+\alpha_{k} r_{0}} \geq \gamma_{k} .
\end{array}
$$

This problem is a variation of the standard QoS problem in (2.3) with mismatched channels and an affine adjustment of the noise power. That connection enables us to analyse the KKT optimality conditions of (2.29) in a similar way to the analysis of Bengtsson and Ottersten (2001); Wiesel et al. (2006); Bjornson et al. (2014) to obtain a quasi-closed-form solution. In particular, if we rewrite the constraint in (2.29b) as $\mathbf{h}_{q_{k}}^{H}\left(\mathbf{w}_{k} \mathbf{w}_{k}^{H} / \gamma_{k}-\sum_{j \neq k} \mathbf{w}_{j} \mathbf{w}_{j}^{H}\right) \mathbf{h}_{q_{k}}-\sigma_{k}^{2} / \alpha_{k} \geq r_{0}$ and let $\nu_{k}$ denote the Lagrange multiplier for this constraint, then we obtain the following characterization 
of the directions

$$
\mathbf{w}_{k}=\left(\frac{\nu_{k}}{\gamma_{k}} \mathbf{h}_{q_{k}} \mathbf{h}_{q_{k}}^{H}-\sum_{j \neq k} \nu_{j} \mathbf{h}_{q_{j}} \mathbf{h}_{q_{j}}^{H}\right) \mathbf{w}_{k},
$$

where the Lagrange multipliers must satisfy the fixed-point relation

$$
\nu_{k}^{-1}=\mathbf{h}_{q_{k}}^{H}\left(\mathbf{I}_{N_{t}}+\sum_{j} \nu_{j} \mathbf{h}_{q_{j}} \mathbf{h}_{q_{j}}^{H}\right)^{-1} \mathbf{h}_{q_{k}}\left(1+\frac{1}{\gamma_{k}}\right) .
$$

To obtain an optimal solution to (2.29), we first find the values for the Lagrange multipliers $\nu_{k}$ in (2.31) using a conventional fixed-point algorithm. Then, the normalized directions $\overline{\mathbf{w}}_{k}=\mathbf{w}_{k} /\left\|\mathbf{w}_{k}\right\|$ can be obtained from (2.30). What then remains to determine is the power allocation to each user, $\beta_{k}=\left\|\mathbf{w}_{k}\right\|^{2}$. Using the fact that at optimality the constraints in (2.29b) hold with equality (Bengtsson and Ottersten, 2001; Wiesel et al., 2006; Bjornson et al., 2014), we obtain a set of linear equations for the vector $\boldsymbol{\beta}=\left[\beta_{1}, \beta_{2}, \ldots, \beta_{K}\right]^{T}$ that takes the form $\mathbf{A} \boldsymbol{\beta}=\mathbf{c}+r_{0} \mathbf{1}$, where $[\mathbf{A}]_{i i}=\left|\mathbf{h}_{q_{i}}^{H} \overline{\mathbf{w}}_{i}\right|^{2} / \gamma_{i},[\mathbf{A}]_{i j}=-\left|\mathbf{h}_{q_{i}}^{H} \overline{\mathbf{w}}_{j}\right|^{2}, \forall i \neq j, \mathbf{c}_{i}=\sigma_{i}^{2} / \alpha_{i}$ and $\mathbf{1}$ is a vector of all ones. Here, $[\mathbf{A}]_{i j}$ denotes the $(i, j)$ th element of the matrix $\mathbf{A}$. Therefore, the solution to (2.29) can be computed by solving that set of equations. That set of equations also provides the connection between (2.27) and (2.29). In particular, since $\sum_{k} \beta_{k}$ is the transmitted power, for an arbitrary $r_{0}>0$, the solution to (2.29) is optimal for (2.27) if $P=\sum_{k} \beta_{k}=\mathbf{1}^{T} \mathbf{A}^{-1} \mathbf{c}+r_{0} \mathbf{1}^{T} \mathbf{A}^{-1} \mathbf{1}$. Since $\mathbf{1}^{T} \mathbf{A}^{-1} \mathbf{c}$ is the power that would be consumed if the CSI was perfect, this expression quantifies the additional power required to provide the robustness specified by $r_{0}$. The inverse of that relation shows that $r^{\star}$, the optimal value in $(2.27)$, is given by $r^{\star}=\left(P-\mathbf{1}^{T} \mathbf{A}^{-1} \mathbf{c}\right) /\left(\mathbf{1}^{T} \mathbf{A}^{-1} \mathbf{1}\right)$.

The expressions obtained above are in a quasi-closed form because they depend on the solution of the $K$ scalar fixed point equations in (2.31). The presence of the 
matrix inversion in (2.31) suggests the use of a Jacobi-style scheme, as it would need only one matrix inversion to be computed in each update cycle of the $\nu_{k}$ 's. The presence of the inverse also suggests the value of obtaining a "good" starting point for the iterations. With that in mind, we observe that if the normalized channel vectors $\mathbf{h}_{q_{k}}$ are nearly orthogonal, $\left(\mathbf{I}_{N_{t}}+\sum_{j} \nu_{j} \mathbf{h}_{q_{j}} \mathbf{h}_{q_{j}}^{H}\right)^{-1} \approx \sum_{j} \frac{1}{1+\nu_{j}} \mathbf{h}_{q_{j}} \mathbf{h}_{q_{j}}^{H}$ and hence $\mathbf{h}_{q_{k}}^{H}\left(\mathbf{I}_{N_{t}}+\sum_{j} \nu_{j} \mathbf{h}_{q_{j}} \mathbf{h}_{q_{j}}^{H}\right)^{-1} \mathbf{h}_{q_{k}} \approx \frac{1}{1+\nu_{j}}$. Employing that approximation in (2.31) we get $\nu_{k}=\gamma_{k}$, and we will use that value as a starting point for the fixed-point equations in (2.31).

An interesting observation from (2.30) and (2.31) is that the beamforming directions depend on the estimates of the channel directions and the SINR targets, but do not depend on the channel gains nor the noise power. On the other hand, the power loading depends on both the channel gains and the noise power in addition to the directions and targets. (Similar observations have been made in the perfect CSI case, Bengtsson and Ottersten, 2001; Wiesel et al., 2006; Bjornson et al., 2014).

\subsubsection{Computational cost}

Employing the quasi-closed-form solution method for the offset maximization design involves three main operations. First, we need to compute $\left\{\nu_{k}\right\}_{k=1}^{K}$ using the fixed-point equations in (2.31). In each of the fixed-point iterations we need to invert $\left(\mathbf{I}_{N_{t}}+\sum_{j} \nu_{j} \mathbf{h}_{q_{j}} \mathbf{h}_{q_{j}}^{H}\right)$ once and for each user we have to pre- and post-multiply that inverse by $\mathbf{h}_{q_{k}}$. As a result, the computational cost per fixed-point iteration is $\mathcal{O}\left(N_{t}^{3}\right)+\mathcal{O}\left(K N_{t}^{2}\right)$. In our numerical experiments reported in Section 2.6, 5 to 15 fixed-point iterations were sufficient to obtain a solution whose outage performance is indistinguishable from that obtained by solving the convex problem in (2.27) using 
the state-of-the-art interior point methods that can be accessed from the CVX tool (Grant et al., 2008). After obtaining $\left\{\nu_{k}\right\}_{k=1}^{K}$, the second step in the quasi-closed-form solution is to solve the eigen equation in (2.30) for each user to obtain the beamforming directions. This requires $\mathcal{O}\left(K N_{t}^{3}\right)$ operations. The third and final step is the power allocation step, which is dominated by the inversion of the matrix $\mathbf{A}$ to find $r^{\star}$ and $\boldsymbol{\beta}$; see the discussion that follows (2.31). Such an inversion can be performed in $\mathcal{O}\left(K^{3}\right)$ operations. In summary, if we let $M$ denote the number of fixed-point iterations, the computational cost of the quasi-closed-form solution contains terms that are $\mathcal{O}\left(M N_{t}^{3}\right), \mathcal{O}\left(M K N_{t}^{2}\right), \mathcal{O}\left(K N_{t}^{3}\right)$ and $\mathcal{O}\left(K^{3}\right)$.

The computational cost of the perfect CSI problem in (2.3), (Bengtsson and Ottersten, 2001; Wiesel et al., 2006; Bjornson et al., 2014), shares the same analysis of that of the offset maximization algorithm above, except for the power allocation part. In the perfect CSI case we solve $\mathbf{A} \boldsymbol{\beta}=\mathbf{c}$, with $r$ set to zero. However, this operation is still dominated by the inversion of the matrix A. According to the previous discussion, we can conclude that the offset maximization algorithm offers a robust precoding design with essentially the same computational cost as that of the conventional perfect CSI problem.

In contrast to the quasi-closed-form solution, the original formulation of the offset maximization problem in (2.27) is a semidefinite program with $K$ LMI constraints of size $N_{t}$. Accordingly, solving this problem using a generic interior point method results in a computational cost per interior-point iteration that is dominated by terms that are $\mathcal{O}\left(K^{3} N_{t}^{6}\right)$ operations (Ye, 2011). The worst-case number of interior-point iterations can be upper bounded by a term that is $\mathcal{O}\left(\sqrt{K N_{t}} \log (1 / \epsilon)\right)$, where $\epsilon$ represents the accuracy of the solution (Ye, 2011). This is a significantly higher computational 
cost than that of the quasi-closed-form solution. Furthermore, the computational costs of generic algorithms for solving the original zero-outage region maximization problem in (2.20) and the constrained offset maximization problem in (2.23) have even higher computational costs, as they share the same $K$ LMI constraints of size $N_{t}$ of the problem in (2.27), and also have additional linear and LMI constraints.

\subsubsection{Channel magnitude effect}

Up until this point, we have considered scenarios in which the dominant quantization errors arise from the quantization of the channel "directions". Hence, we have assumed that the BS has accurate knowledge of the channel gains $\alpha_{k}$. To examine the effect of quantizing $\alpha_{k}$ we rewrite the expression in (2.11) as

$$
\operatorname{SINR}_{k}\left(\mathbf{e}_{k}\right)=\frac{\left(\mathbf{h}_{q_{k}}+\mathbf{e}_{k}\right)^{H} \mathbf{W}_{k}\left(\mathbf{h}_{q_{k}}+\mathbf{e}_{k}\right)}{\left(\mathbf{h}_{q_{k}}+\mathbf{e}_{k}\right)^{H}\left(\sum_{j \neq k} \mathbf{W}_{j}\right)\left(\mathbf{h}_{q_{k}}+\mathbf{e}_{k}\right)+\sigma_{k}^{2} / \alpha_{k}} .
$$

This expression shows that the quantity to be quantized is actually the noise variance to the power gain ratio, $\sigma_{k}^{2} / \alpha_{k}$. It also suggests that in scenarios in which the interference dominates the noise, which are common in practice, the effects of quantizing $\sigma_{k}^{2} / \alpha_{k}$ using a scalar codebook will be small. Indeed, as discussed at the end of the previous subsection, it can be seen from (2.30) and (2.31) that the beamforming directions $\overline{\mathbf{w}}_{k}$ are independent of $\sigma_{k}^{2} / \alpha_{k}$. It is only the power allocation that is dependent on this ratio. As we will illustrate in Section 2.6, in interference limited scenarios even if the BS has no knowledge of $\sigma_{k}^{2} / \alpha_{k}$ and removes this term from (2.27c), the resulting beamformers still provide good performance. 


\subsection{Extensions to TDD case}

In this section, we show how the analysis in the previous section, which was developed for FDD systems in which the dominant uncertainties are those of channel directions, can be adapted to TDD systems in which the dominant uncertainties are uplink estimation errors that can be modelled as being Gaussian. In that case, the uncertainty model takes the form in (2.10), and we will focus on the case in which the elements of $\mathbf{e}_{k}$ are uncorrelated and have the same variance. In that case, a sphere of radius $\varepsilon$ is the appropriate shape for the zero-outage region, and the semidefinite relaxation of the problem in (2.6) of power minimization subject to a zero-outage constraint can be written as (Wang et al., 2014)

$$
\begin{aligned}
\min _{\mathbf{W}_{k}, \lambda_{k}} & \sum_{k=1}^{K} \operatorname{tr}\left(\mathbf{W}_{k}\right) \\
\text { s.t. } & \left(\begin{array}{cc}
\mathbf{Q}_{k} & \mathbf{Q}_{k} \mathbf{h}_{e_{k}} \\
\mathbf{h}_{e_{k}}^{H} \mathbf{Q}_{k} & \mathbf{h}_{e_{k}}^{H} \mathbf{Q}_{k} \mathbf{h}_{e_{k}}-\sigma_{k}^{2}
\end{array}\right)+\lambda_{k}\left(\begin{array}{cc}
\mathbf{I} & \mathbf{0} \\
\mathbf{0} & -\varepsilon^{2}
\end{array}\right) \succeq \mathbf{0 .} . \\
& \mathbf{W}_{k} \succeq \mathbf{0}, \quad \lambda_{k} \geq 0 .
\end{aligned}
$$

Similarly, the SDR of the problem in (2.7) of maximizing the volume of the zerooutage region subject to a power constraint can be written as

$$
\begin{aligned}
\max _{\mathbf{W}_{k}, \lambda_{k}, \varepsilon} & \varepsilon \\
\text { subject to } & \sum_{k=1}^{K} \operatorname{tr}\left(\mathbf{W}_{k}\right) \leq p \\
& \mathbf{F}_{0 k}\left(\mathbf{Q}_{k}\right)+\lambda_{k} \mathbf{F}_{1}(\varepsilon) \succeq \mathbf{0} \\
& \mathbf{W}_{k} \succeq \mathbf{0}, \quad \lambda_{k} \geq 0,
\end{aligned}
$$


where $\mathbf{F}_{0 k}\left(\mathbf{Q}_{k}\right)$ and $\mathbf{F}_{1}(\varepsilon)$ were defined after (2.18).

As discussed earlier, the weakness of the formulation in (2.7), and hence that in (2.34), is that the performance of the system under certainties outside the zero-outage region is not considered. By extending the concept of offset maximization developed in Section 2.4.3 to the TDD case, we obtain the following variant of the problem in

$$
\begin{array}{rl}
\max _{\mathbf{W}_{k} \succeq \mathbf{0}, r} & r \\
\text { subject to } & \sum_{k} \operatorname{tr}\left(\mathbf{W}_{k}\right) \leq P, \\
& \mathbf{h}_{e_{k}}^{H} \mathbf{Q}_{k} \mathbf{h}_{e_{k}}-\sigma_{k}^{2}-r \geq 0 .
\end{array}
$$

By making some straightforward modifications to the analysis in Sections 2.4.3 and 2.4.4, it can be be shown that the problem in (2.35) always has an optimal solution that has rank one and such a solution can be found in a quasi-closed form.

\subsection{Simulation studies}

In this section we provide simulation results that demonstrate the performance of the proposed designs, and, in particular, the designs based on the notion of offset maximization; cf. (2.27) and (2.35). We consider a single-cell downlink system in which the BS has four antennas and three single-antenna users served concurrently. An independent Rayleigh fading channel model is used, with the channel from each BS antenna to each user is modelled as being independent with a circular complex Gaussian distribution with zero mean and unit variance. The additive noise at each receiver has a variance $\sigma_{k}^{2}=0.01$. 


\subsubsection{FDD systems}

To study the performance of the algorithms proposed for FDD systems, we consider a Grassmanian feedback scheme (Love et al., 2003) in which each user employs a randomly rotated version of a single 13-bit codebook. That codebook was selected from a set of codebooks that was generated randomly using the isotropic distribution on the manifold. The size of the error $\mathbf{e}_{k}$ is assessed in terms of the Grassmanian distance between the quantized and the true channels; i.e., $d\left(\mathbf{e}_{k}\right)=\min _{\theta}\left\|\mathbf{h}_{k} e^{j \theta} / \sqrt{\alpha_{k}}-\mathbf{h}_{q_{k}}\right\|$.

To illustrate the basic properties of the proposed scheme, we first consider a synthetic example that enables us to visualize the statistics of the quantization errors. In this example we first picked, at random, three elements of the codebook as the quantized directions for the three users. Then we generated a sufficient number of Rayleigh channel realizations so that at least 5000 channel realizations were quantized to each of the three selected elements of the codebook. The empirical distribution of the Grassmanian distances of those channel realizations from the corresponding codebook element is plotted as the lower curve with the right hand axis in Fig. 2.1. Next, using the quantized channels, we designed a variety of robust beamformers for a system with a transmission power constraint of $P=1$ and an SINR target for each user of $\gamma_{k}=9 \mathrm{~dB}$. The design methods we considered are the zero-outage region maximization design (ZOR max.) in (2.20), the constrained offset maximization design (Constr. offset max.) in (2.26) with a value of the zero-outage region size $\varepsilon_{0}=(5 / 6) \varepsilon^{\star}$, where $\varepsilon^{\star}$ is the optimal value for the zero-outage region maximization design in (2.20), and the quasi-closed-form solution developed in Section 2.4.4 for the offset maximization (Offset max.) problem in (2.27). Those designs were then evaluated on the actual channels that were quantized to the chosen $\mathbf{h}_{q_{k}}$. In the upper 


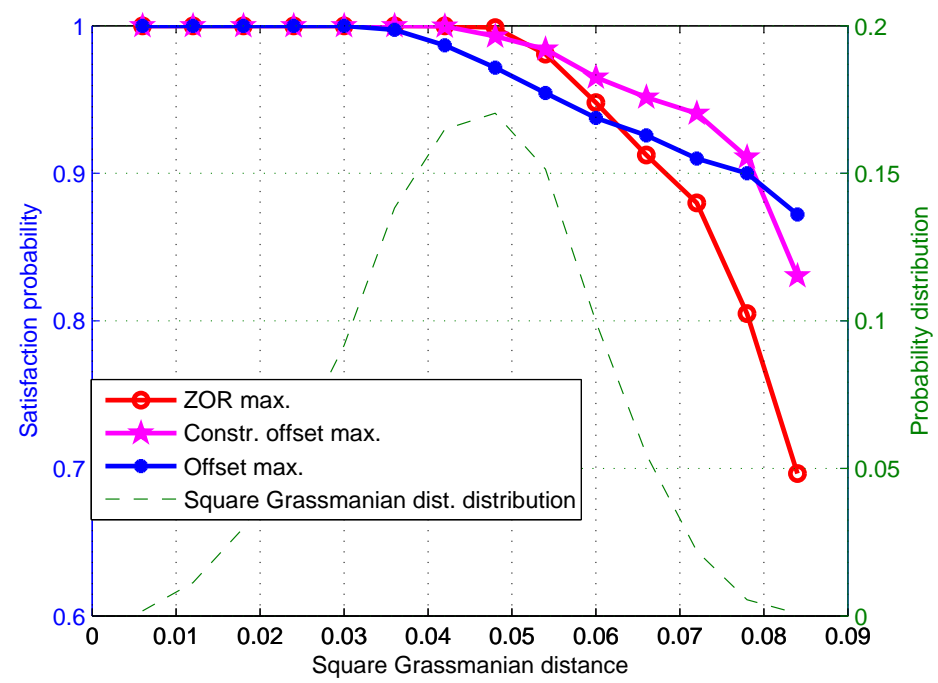

Figure 2.1: Satisfaction probability $=(1$-outage probability $)$ for the synthetic example involving an FDD system with 4 BS antennas, 3 users, and SINR targets $\gamma_{k}=9 \mathrm{~dB}$.

curves of Fig. 2.1, we have plotted the probability that each of the considered design methods satisfies the SINR constraints against the size of the squared Grassmanian distances. These results outline the basic tradeoffs between the three proposed algorithms. By design, the method that maximizes the zero-outage region maintains zero-outage for larger uncertainties than the other two designs, but its performance degrades rapidly as the size of uncertainty grows beyond that point. The constrained offset maximization design trades a small degradation in the size of the zero-outage region for better performance under large uncertainties. The offset maximization approach does not seek a zero-outage region, but provides improved robustness to large uncertainties, and does so at a much lower computational cost than the other methods.

Having illustrated the principles of the approaches through the results in Fig. 2.1 for a synthetic experiment, we now consider the practical performance of the proposed 
schemes. We will include as benchmarks the performance of the conventional mismatched designs based on $\mathrm{ZF}$ and $\mathrm{RCI}$ with the regularization coefficient of $N_{t} \sigma_{k}^{2} / P$ suggested by Peel et al. (2005), and the mismatched design based on solving (2.3) under the assumption that the estimated CSI is perfect (cf. Bjornson et al., 2014). In the experiments, the receivers are assumed to identify the channels with sufficient accuracy so that the quantized error incurred by using the Grassmanian codebooks at each user are the dominant errors. The precoders were designed using the quantized channel directions and the SINR of each user was evaluated using the actual channels. The outage probability was estimated by repeating the experiment over 8,000 channel realizations. In Figs 2.2 and 2.3 we have plotted the outage probability as a function of the bound on the transmitted power, where in the case of the conventional ZF, RCI and "perfect CSI" precoders, the precoding vectors are scaled to match the power constraint. In Fig. 2.2 the target SINR was $\gamma_{k}=3 \mathrm{~dB}$ and in Fig. 2.3 it was $\gamma_{k}=6 \mathrm{~dB}$. We observe that the proposed methods provide significantly lower outage probability than the existing techniques, and that when the SINR targets are more aggressive (i.e., in Fig. 2.3 relative to Fig. 2.2, or at lower transmission power in either Fig. 2.2 or 2.3), the offset maximization design provides significantly better performance than the zero-outage region maximization design. (Recall that the offset maximization design also has the advantage that it has a quasi-closed-form solution.)

The solid curves in Figs 2.2 and 2.3 illustrate the performance of the various algorithms with perfect knowledge of the gains $\alpha_{k}$ and the noise variance $\sigma_{k}^{2}$. Actually, as mentioned in Section 2.4.6, all that is needed is $\sigma_{k}^{2} / \alpha_{k}$. To assess the sensitivity of the proposed offset maximization algorithm to errors in quantizing $\sigma_{k}^{2} / \alpha_{k}$, the dashed curves in Figs 2.2 and 2.3 show the performance of the algorithm in the extreme 


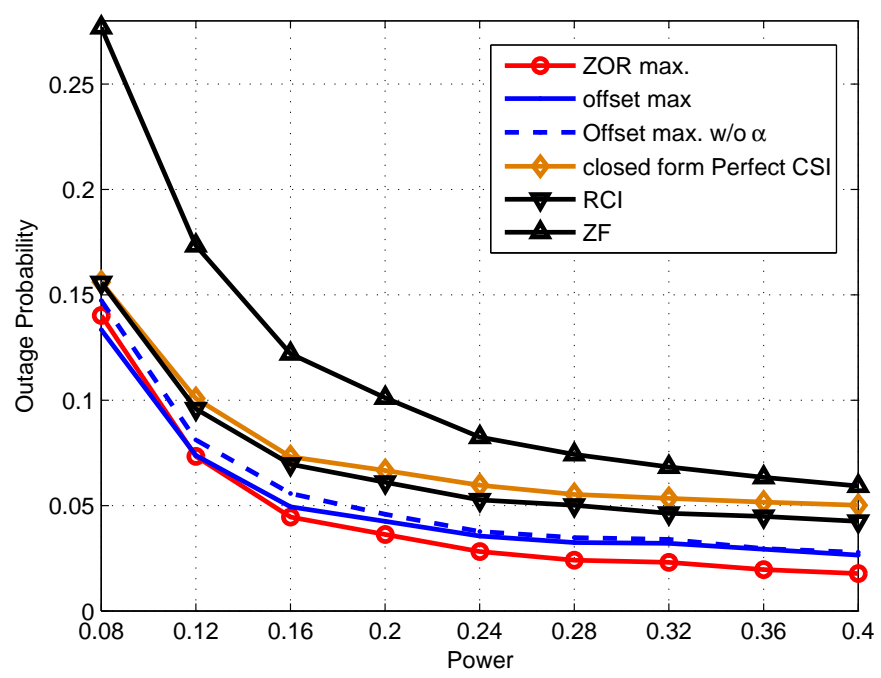

Figure 2.2: Outage probability for an FDD system with 4 BS antennas, 3 users, and SINR targets $\gamma_{k}=3 \mathrm{~dB}$

case in which the BS has no knowledge of $\sigma_{k}^{2} / \alpha_{k}$. In that case, the beamformers are designed using the variant of (2.27) in which $\sigma_{k}^{2} / \alpha_{k}$ is removed from $(2.27 \mathrm{c})$. The fact that the dashed curves are close to the corresponding solid curves confirms the analysis in Section 2.4.6, which suggests that in interference limited scenarios the proposed design is quite robust to the quantization of $\sigma_{k}^{2} / \alpha_{k}$.

\subsubsection{TDD systems}

To illustrate the performance of the variant of the offset maximization algorithm for TDD systems, we consider the same physical scenario as in the previous experiments, but now the channel uncertainty takes the form of an additive zero-mean circular Gaussian random vector with covariance $0.005 \mathbf{I}_{N_{t}}$. The performance of the offset maximization is compared to that of the sphere bounding approach of Wang et al. 


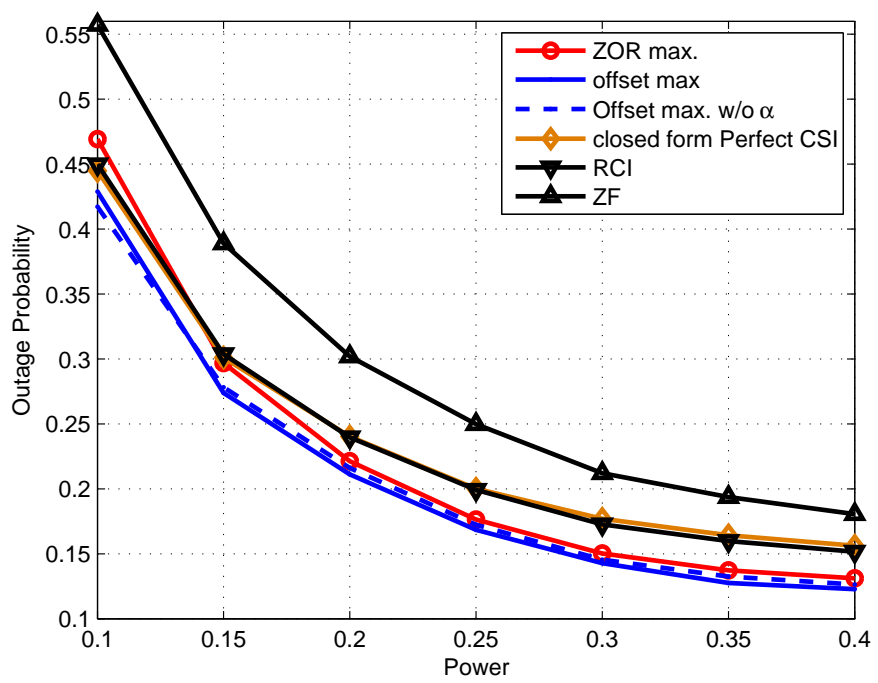

Figure 2.3: Outage probability for an FDD system with 4 BS antennas, 3 users, and SINR targets $\gamma_{k}=6 \mathrm{~dB}$

(2014) (SB algorithm), and the conventional RCI approach. Although the relative performance gains in Fig. 2.4 are smaller than those for the FDD case, the proposed offset maximization design provides better performance than the SB and RCI approaches and has a quasi-closed-form solution.

\subsection{Conclusion}

Although various approaches for incorporating robustness to channel uncertainty in the design of downlink beamforming schemes are available, none of them is based on uncertainty models that reflect the nature of the errors that arise in systems with limited feedback. In Section 2.3 we derived a variant of the S-Lemma for complexvalued systems that enabled the development of such a scheme. The resulting design 


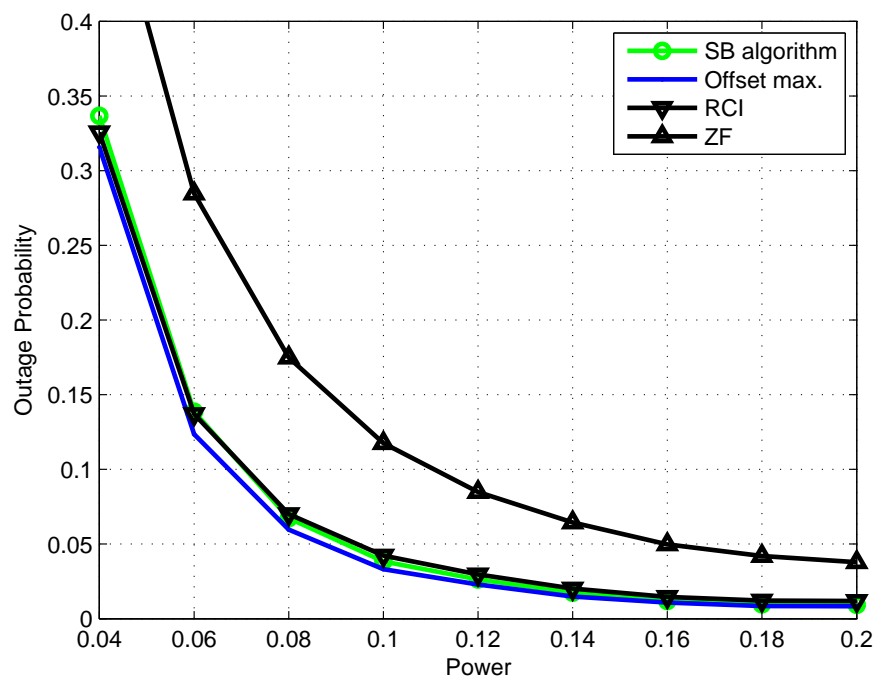

Figure 2.4: Outage probability for a TDD system with 4 BS antennas, 3 users, and SINR targets $\gamma_{k}=3 \mathrm{~dB}$

approach provides excellent performance for small uncertainties. However, as has typically been the case for the existing approaches, its conservatism results in degraded performance for larger uncertainties, and it imposes a significantly larger computational burden on the base station than conventional mismatched designs (in which the channel estimate is employed as if it were precise). In Section 2.4.3, we used insights into the structure of the proposed scheme for limited feedback systems to develop an alternate design formulation that provides greater robustness in the presence of larger uncertainties, and has a quasi-closed-form solution. Simulation results confirmed these theoretical insights and suggest that highly effective robust designs can be obtained with essentially the same computational effort as conventional designs. 


\section{A Appendix: Proof of Theorem 1}

Our first step is to prove a homogeneous version of Theorem 1.

Lemma 1 Let

$$
\mathbf{F}_{i}=\left(\begin{array}{cc}
\mathbf{A}_{i} & \mathbf{b}_{i} \\
\mathbf{b}_{i}^{H} & c_{i}
\end{array}\right) \in \mathbb{H}^{(n+1) \times(n+1)}, i=0,1,2,
$$

suppose that $\mathbf{F}_{2}$ is indefinite, and that there exists $\mathbf{y}_{0} \in \mathbb{C}^{n+1}$ such that $\mathbf{y}_{0}^{H} \mathbf{F}_{1} \mathbf{y}_{0}<0$ and $\mathbf{y}_{0}^{H} \mathbf{F}_{2} \mathbf{y}_{0}=0$. Then the following two statements are equivalent:

1. $\mathbf{y}^{H} \mathbf{F}_{0} \mathbf{y} \geq 0$ for all $\mathbf{y}$ satisfying $\mathbf{y}^{H} \mathbf{F}_{1} \mathbf{y} \leq 0$ and $\mathbf{y}^{H} \mathbf{F}_{2} \mathbf{y}=0$.

2. There exists $\lambda \geq 0, \mu \in \mathbb{R}$ such that

$$
\mathbf{F}_{0}+\lambda \mathbf{F}_{1}+\mu \mathbf{F}_{2} \succeq \mathbf{0} .
$$

Proof of Lemma 1: To establish the implication from Statement 2 to Statement 1 we observe that for any $\mathbf{y}$ satisfying $\mathbf{y}^{H} \mathbf{F}_{1} \mathbf{y} \leq 0$ and $\mathbf{y}^{H} \mathbf{F}_{2} \mathbf{y}=0$ and for any $\lambda \geq 0, \mu \in \mathbb{R}$ satisfying (2.37), one has

$$
\mathbf{y}^{H} \mathbf{F}_{0} \mathbf{y} \geq \mathbf{y}^{H}\left(\mathbf{F}_{0}+\lambda \mathbf{F}_{1}+\mu \mathbf{F}_{2}\right) \mathbf{y} \geq 0
$$


The implication from Statement 1 to Statement 2 is proved as follows. Consider the semidefinite program (SDP):

$$
\begin{aligned}
\min _{\mathbf{X}} & \operatorname{tr}\left(\mathbf{F}_{0} \mathbf{X}\right) \\
\text { subject to } & \operatorname{tr}\left(\mathbf{F}_{1} \mathbf{X}\right) \leq 0, \operatorname{tr}\left(\mathbf{F}_{2} \mathbf{X}\right)=0, \\
& \operatorname{tr}(\mathbf{X})=1, \\
& \mathbf{X} \succeq \mathbf{0},
\end{aligned}
$$

where $\mathbf{X} \in \mathbb{H}^{\left(N_{t}+1\right) \times\left(N_{t}+1\right)}$. The (Lagrangian) dual problem of (2.38) is

$$
\begin{aligned}
\max _{\lambda, \mu, \nu} & \nu \\
\text { subject to } & \mathbf{F}_{0}+\lambda \mathbf{F}_{1}+\mu \mathbf{F}_{2}-\nu \mathbf{I}_{N_{t}+1} \succeq \mathbf{0}, \\
& \lambda \geq 0,
\end{aligned}
$$

where $\lambda, \mu, \nu \in \mathbb{R}$. Let $p^{\star}$ and $\nu^{\star}$ denote the optimal values of the problems in (2.38) and (2.39), respectively. Suppose that the problems in (2.38) and (2.39) are both strictly feasible. Then, strong duality holds (i.e., $p^{\star}=d^{\star}$ ), the (shared) optimal value is finite, and in both problems the optimal value is attained (Ben-Tal and Nemirovski, 2001, Theorem 2.4.1, Corollary 2.4.1). Therefore, using a specific rankone decomposition theorem (Huang and Zhang, 2007; Sturm and Zhang, 2003), there exists a rank-one solution to problem (2.38). ${ }^{1}$ Let us denote that rank-one solution by $\mathbf{Y}^{\star}=\mathbf{y}^{\star} \mathbf{y}^{\star H}$, where $\mathbf{y}^{\star} \in \mathbb{C}^{n+1}$. If Statement 1 holds, then we have that

$$
0 \leq \mathbf{y}^{\star H} \mathbf{F}_{0} \mathbf{y}^{\star}=p^{\star}=\nu^{\star} .
$$

\footnotetext{
${ }^{1}$ Alternatively, the existence of a rank-one solution can be established using the SDP rank reduction procedure of Huang and Palomar (2010) or the randomized postprocessing procedure of Huang and Palomar (2014).
} 
Let $\left(\lambda^{\star}, \mu^{\star}, \nu^{\star}\right)$ denote the solution to the problem in (2.39). Eq. (2.40) implies that

$$
\mathbf{F}_{0}+\lambda^{\star} \mathbf{F}_{1}+\mu^{\star} \mathbf{F}_{2} \succeq \nu^{\star} \mathbf{I}_{N_{t}+1} \succeq \mathbf{0} .
$$

Hence, the choice of $\lambda^{\star}$ and $\mu^{\star}$ satisfies Statement 2 .

The above argument is based on the supposition that the problems in (2.38) and (2.39) are both strictly feasible. To complete the proof we need to establish that that is indeed the case. To verify the strict feasibility of (2.39) we can choose a small $\lambda>0$, choose $\mu=0$ and then find a negative value of $\nu$ with a magnitude that is large enough for $\mathbf{F}_{0}+\lambda \mathbf{F}_{1}+\mu \mathbf{F}_{2}-\nu \mathbf{I}_{N_{t}+1} \succ \mathbf{0}$. To establish the strict feasibility of (2.38) we use the following argument. First, since $\mathbf{F}_{2}$ is indefinite, we can find $\mathbf{Y}_{0} \succ \mathbf{0}$ such that $\operatorname{tr}\left(\mathbf{F}_{2} \mathbf{Y}_{0}\right)=0$. Now, recall from the statement of Lemma 1 that there exists $\mathbf{y}_{0}$ such that $\mathbf{y}_{0}^{H} \mathbf{F}_{1} \mathbf{y}_{0}<0$ and $\mathbf{y}_{0}^{H} \mathbf{F}_{2} \mathbf{y}_{0}=0$. Using $\mathbf{Y}_{0}, \mathbf{y}_{0}$, and an $\epsilon \in(0,1)$ construct

$$
\mathbf{X}(\epsilon)=(1-\epsilon) \frac{1}{\left\|\mathbf{y}_{0}\right\|^{2}} \mathbf{y}_{0} \mathbf{y}_{0}^{H}+\frac{\epsilon}{\operatorname{tr}\left(\mathbf{Y}_{0}\right)} \mathbf{Y}_{0}
$$

It can be verified that for any $\epsilon \in(0,1), \mathbf{X}(\epsilon) \succ \mathbf{0}, \operatorname{tr}(\mathbf{X}(\epsilon))=1$, and $\operatorname{tr}\left(\mathbf{F}_{2} \mathbf{X}(\epsilon)\right)=$ 0. Also, it can be checked that for a sufficiently small $\epsilon>0$, it holds true that $\operatorname{tr}\left(\mathbf{F}_{1} \mathbf{X}(\epsilon)\right)<0$. Therefore, the problem in (2.38) is strictly feasible.

Having established Lemma 1, we are now ready to prove Theorem 1 . We divide the proof into two steps.

Step 1: Let

$$
\begin{array}{r}
\mathcal{A}=\left\{\mathbf{F}_{0} \mid \mathbf{y}^{H} \mathbf{F}_{0} \mathbf{y} \geq 0, \quad \forall \mathbf{y} \text { such that } \mathbf{y}^{H} \mathbf{F}_{1} \mathbf{y} \leq 0, \mathbf{y}^{H} \mathbf{F}_{2} \mathbf{y}=0\right\}, \\
\mathcal{A}_{1}=\left\{\mathbf{F}_{0} \mid f_{0}(\mathbf{x}) \geq 0, \forall \mathbf{x} \text { such that } f_{1}(\mathbf{x}) \leq 0, f_{2}(\mathbf{x})=0\right\} .
\end{array}
$$


Suppose that $\mathcal{A}$ and $\mathcal{A}_{1}$ are equivalent. Then, using Lemma 1, the two statements in Theorem 1 can be shown to be equivalent. Specifically, given a vector $\mathbf{y} \in \mathbb{C}^{n+1}$, partition

$$
\mathbf{y}=\left(\begin{array}{c}
\mathbf{x} \\
t
\end{array}\right),
$$

and note that

$$
\mathbf{y}^{H} \mathbf{F}_{i} \mathbf{y}=|t|^{2} f_{i}(\mathbf{x} / t), i=0,1,2,
$$

whenever $t \neq 0$. Suppose that $\mathbf{F}_{0} \in \mathcal{A}_{1}$, and that there exists $\mathbf{x}_{0}$ such that $f_{1}\left(\mathbf{x}_{0}\right)<0$ and $f_{2}\left(\mathbf{x}_{0}\right)=0$. By denoting

$$
\mathbf{y}_{0}=\left(\begin{array}{c}
\mathbf{x}_{0} \\
1
\end{array}\right),
$$

we see that $\mathbf{y}_{0}^{H} \mathbf{F}_{1} \mathbf{y}_{0}=f_{1}\left(\mathbf{x}_{0}\right)<0$ and $\mathbf{y}_{0}^{H} \mathbf{F}_{2} \mathbf{y}_{0}=f_{2}\left(\mathbf{x}_{0}\right)=0$. Consequently we can apply Lemma 1 to show that

$$
\begin{gathered}
\exists \lambda \geq 0, \mu \text { such that } \mathbf{F}_{0}+\lambda \mathbf{F}_{1}+\mu \mathbf{F}_{2} \succeq \mathbf{0} \\
\Longleftrightarrow \mathbf{y}^{H} \mathbf{F}_{0} \mathbf{y} \geq 0, \forall \mathbf{y} \text { such that } \mathbf{y}^{H} \mathbf{F}_{1} \mathbf{y} \leq 0, \mathbf{y}^{H} \mathbf{F}_{2} \mathbf{y}=0 \\
\Longleftrightarrow f_{0}(\mathbf{x}) \geq 0, \forall \mathbf{x} \text { such that } f_{1}(\mathbf{x}) \leq 0, f_{2}(\mathbf{x})=0,
\end{gathered}
$$

where the second equivalence follows from the assumption that $\mathcal{A}=\mathcal{A}_{1}$.

Step 2: To complete the proof we now establish the equivalence $\mathcal{A}=\mathcal{A}_{1}$. From (2.41)-(2.42) one can readily see that any $\mathbf{F}_{0} \in \mathcal{A}$ also lies in $\mathcal{A}_{1}$. (In particular, set $t=1$.) Thus, let us focus on the converse; i.e., $\mathbf{F}_{0} \in \mathcal{A}_{1} \Longrightarrow \mathbf{F}_{0} \in \mathcal{A}$. Suppose $\mathbf{F}_{0} \in \mathcal{A}_{1}$. We examine two cases, namely, i) $t \neq 0$ and ii) $t=0$. 
For the first case, we see from (2.42) that for any $\mathbf{y}$ satisfying $t \neq 0, \mathbf{y}^{H} \mathbf{F}_{1} \mathbf{y} \equiv$ $|t|^{2} f_{1}(\mathbf{x} / t) \leq 0$ and $\mathbf{y}^{H} \mathbf{F}_{2} \mathbf{y} \equiv|t|^{2} f_{2}(\mathbf{x} / t)=0$, and we have that $\mathbf{y}^{H} \mathbf{F}_{0} \mathbf{y}=|t|^{2} f_{0}(\mathbf{x} / t) \geq$ 0 .

For the second case, consider any

$$
\mathbf{y}=\left(\begin{array}{l}
\mathbf{x} \\
0
\end{array}\right)
$$

that satisfies $\mathbf{y}^{H} \mathbf{F}_{1} \mathbf{y} \leq 0$ and $\mathbf{y}^{H} \mathbf{F}_{2} \mathbf{y}=0$.

We wish to show that $\mathbf{y}^{H} \mathbf{F}_{0} \mathbf{y} \geq 0$. To do so, let $\mathbf{x}_{0}$ be an arbitrary vector satisfying $f_{1}\left(\mathbf{x}_{0}\right) \leq 0$ and $f_{2}\left(\mathbf{x}_{0}\right)=0$, which must exist, due to the fact that $\mathbf{F}_{0} \in \mathcal{A}_{1}$. From $\mathbf{x}_{0}$ we construct $\mathbf{y}_{0}$ using (2.43), and note that the corresponding $\mathbf{y}_{0}$ satisfies $\mathbf{y}_{0}^{H} \mathbf{F}_{1} \mathbf{y}_{0} \leq 0$ and $\mathbf{y}_{0}^{H} \mathbf{F}_{2} \mathbf{y}_{0}=0$. Also, let

$$
\mathbf{y}(\epsilon)=\mathbf{y}+\epsilon \mathbf{y}_{0},
$$

where $\epsilon=r e^{j \theta} \neq 0$ (i.e., $r>0$ ). We have

$$
\mathbf{y}(\epsilon)^{H} \mathbf{F}_{1} \mathbf{y}(\epsilon)=|\epsilon|^{2} \mathbf{y}_{0}^{H} \mathbf{F}_{1} \mathbf{y}_{0}+2 \operatorname{Re}\left\{\epsilon \mathbf{y}^{H} \mathbf{F}_{1} \mathbf{y}_{0}\right\}+\mathbf{y}^{H} \mathbf{F}_{1} \mathbf{y} \leq 2 \operatorname{Re}\left\{\epsilon \mathbf{y}^{H} \mathbf{F}_{1} \mathbf{y}_{0}\right\},
$$

and

$$
\begin{aligned}
\mathbf{y}(\epsilon)^{H} \mathbf{F}_{2} \mathbf{y}(\epsilon) & =|\epsilon|^{2} \mathbf{y}_{0}^{H} \mathbf{F}_{2} \mathbf{y}_{0}+2 \operatorname{Re}\left\{\epsilon \mathbf{y}^{H} \mathbf{F}_{2} \mathbf{y}_{0}\right\}+\mathbf{y}^{H} \mathbf{F}_{2} \mathbf{y} \\
& =2 \operatorname{Re}\left\{\epsilon \mathbf{y}^{H} \mathbf{F}_{2} \mathbf{y}_{0}\right\} .
\end{aligned}
$$

Let $\mathbf{y}^{H} \mathbf{F}_{i} \mathbf{y}_{0}=r_{i} e^{j \theta_{i}}, i=1,2$. Then,

$$
\mathbf{y}(\epsilon)^{H} \mathbf{F}_{1} \mathbf{y}(\epsilon) \leq 2 r r_{1} \operatorname{Re}\left\{e^{j\left(\theta+\theta_{1}\right)}\right\}
$$


and

$$
\mathbf{y}(\epsilon)^{H} \mathbf{F}_{2} \mathbf{y}(\epsilon)=2 r r_{2} \operatorname{Re}\left\{e^{j\left(\theta+\theta_{2}\right)}\right\}
$$

Observing (2.47) and (2.48), we can take the value of $\theta$ as either $\bar{\theta}=\frac{\pi}{2}-\theta_{2}$ or $\bar{\theta}=-\frac{\pi}{2}-\theta_{2}$ such that $\operatorname{Re}\left\{e^{j\left(\bar{\theta}+\theta_{2}\right)}\right\}=0$ and $\operatorname{Re}\left\{e^{j\left(\bar{\theta}+\theta_{1}\right)}\right\} \leq 0$. Hence we have

$$
\mathbf{y}\left(r e^{j \bar{\theta}}\right)^{H} \mathbf{F}_{1} \mathbf{y}\left(r e^{j \bar{\theta}}\right) \leq 0 \text { and } \mathbf{y}\left(r e^{j \bar{\theta}}\right)^{H} \mathbf{F}_{2} \mathbf{y}\left(r e^{j \bar{\theta}}\right)=0
$$

for any $r>0$. By also noting that the last component of $\mathbf{y}\left(r e^{j \bar{\theta}}\right)$ is nonzero, it follows from the first case that

$$
\mathbf{y}\left(r e^{j \bar{\theta}}\right)^{H} \mathbf{F}_{0} \mathbf{y}\left(r e^{j \bar{\theta}}\right) \geq 0
$$

for any $r>0$. By letting $r \rightarrow 0$, one has $\mathbf{y}\left(r e^{j \bar{\theta}}\right) \rightarrow \mathbf{y}$ and $\mathbf{y}^{H} \mathbf{F}_{0} \mathbf{y} \geq 0$.

By combining the above two cases, we conclude that $\mathbf{F}_{0} \in \mathcal{A}$ whenever $\mathbf{F}_{0} \in \mathcal{A}_{1}$. Thus, the proof of Theorem 1 is complete.

\section{B Appendix: Bound derivation}

We will establish the existence of rank-one solutions for sufficiently small $\varepsilon$ by adapting the analysis of Song et al. (2012) to the problem at hand. To begin, let $\mathbf{U}_{k}=$ $\left(\begin{array}{cc}\mathbf{Y}_{k} & \mathbf{y}_{k} \\ \mathbf{y}_{k}^{H} & \zeta_{k}\end{array}\right) \in \mathbb{H}^{(n+1) \times(n+1)}$ denote the dual variable associated with the $k$ th constraint in (2.19b), and let $\mathbf{Z}_{k} \in \mathbb{H}^{n \times n}$ and $\eta_{k}$ denote the dual variables associated with the constraints in (2.19c), respectively. In addition, let $P^{\star}$ denote the optimal value for 
the problem in (2.19). Then the Lagrangian dual of (2.19) can be written as

$$
\begin{aligned}
\max _{\mathbf{Y}_{k}, \mathbf{y}_{k}, \zeta_{k}} & \sum_{k=1}^{K} \zeta_{k} \sigma_{k}^{2} / \alpha_{k} \\
\text { s.t. } & \eta_{k} \triangleq \zeta_{k} \varepsilon^{2}-\operatorname{tr}\left(\mathbf{Y}_{k}\right) \geq 0, \\
& \mathbf{U}_{k} \triangleq\left(\begin{array}{cc}
\mathbf{Y}_{k} & \mathbf{y}_{k} \\
\mathbf{y}_{k}^{H} & \zeta_{k}
\end{array}\right) \succeq \mathbf{0}, \\
& \mathbf{Z}_{k} \triangleq \mathbf{I}_{n}-\frac{1}{\gamma_{k}}\left(\mathbf{Y}_{k}+\mathbf{y}_{k} \mathbf{h}_{q_{k}}^{H}+\mathbf{h}_{q_{k}} \mathbf{y}_{k}^{H}+\zeta_{k} \mathbf{h}_{q_{k}} \mathbf{h}_{q_{k}}^{H}\right) \\
& +\sum_{j \neq k}\left(\mathbf{Y}_{j}+\mathbf{y}_{j} \mathbf{h}_{q_{j}}^{H}+\mathbf{h}_{q_{j}} \mathbf{y}_{j}^{H}+\zeta_{j} \mathbf{h}_{q_{j}} \mathbf{h}_{q_{j}}^{H}\right) \succeq \mathbf{0}, \\
& \operatorname{tr}\left(\mathbf{Y}_{k}+\mathbf{y}_{k} \mathbf{h}_{q_{k}}^{H}+\mathbf{h}_{q_{k}} \mathbf{y}_{k}^{H}\right)=0 .
\end{aligned}
$$

If we were to remove (2.49e), then (2.49) would become the same as the dual problem studied by Song et al. (2012); cf. $\left(D_{\epsilon}\right)$ in (Song et al., 2012). Hence we can adopt the same principles employed therein to identify instances where (2.49a)-(2.49d) can be utilized to imply that the primal problem (2.19) has a rank-one solution. Doing so shows that if $\varepsilon^{2} \leq \min _{k} \frac{\sigma_{k}^{2} \gamma_{k}}{P^{\star} \alpha_{k}}$, then there is an optimal solution to (2.19) in which each $\mathbf{W}_{k}^{\star}$ has rank one. It is difficult to use this result to predict whether a particular instance of the problem in (2.19) is guaranteed to have an optimal solution that has rank one, because the bound depends on $P^{\star}$. However, the bound does demonstrate that for sufficiently small $\varepsilon$ there is a rank-one optimal solution. 


\section{C Appendix: Offset-maximization algorithm}

In this appendix we establish the fact that as $\varepsilon_{0} \rightarrow 0$ any optimal set for (2.26) approaches an optimal set for (2.23). As a first step, we observe that any feasible point for the problem in (2.23), including the optimum point, is a feasible point for (2.26). This is due to the fact that the condition in (2.26c) is a necessary condition for the LMIs in (2.23c) to hold. Next, we consider the following variant of (2.26)

$$
\begin{array}{rl}
\max _{\mathbf{W}_{k}, r_{k}, t} & t \\
\text { subject to } & \sum_{k} \operatorname{tr}\left(\mathbf{W}_{k}\right) \leq P, \\
& \mathbf{h}_{q_{k}}^{H} \mathbf{Q}_{k} \mathbf{h}_{q_{k}}-\sigma_{k}^{2} / \alpha_{k}-r_{k} \geq \delta, \\
& \mathbf{W}_{k} \succeq \mathbf{0}, \quad r_{k} \geq t, \quad t \geq 0 .
\end{array}
$$

where $\delta$ is a small positive number. Given the optimal solution to (2.50), denoted $\left\{\mathbf{W}_{k}^{\star}\right\},\left\{r_{k}^{\star}\right\}, t^{\star}$, we can construct $\lambda_{k}=r_{k}^{\star} / \varepsilon_{0}^{2}$. To establish the connection between (2.23) and (2.26), we will show that as $\varepsilon_{0} \rightarrow 0$, for any $\delta>0$ there exists a set $\left\{\mu_{k}\right\}$ such that the collection $\left\{\mathbf{W}_{k}^{\star}\right\},\left\{\lambda_{k}\right\}, t^{\star}$ and $\left\{\mu_{k}\right\}$ represents a feasible point for (2.23).

To establish this fact, we apply the theory of Schur complements to the LMI condition in (2.23c). This LMI takes the form $\left(\begin{array}{cc}\mathbf{A} & \mathbf{b} \\ \mathbf{b}^{H} & c\end{array}\right) \succeq \mathbf{0}$, and a sufficient condition for the LMI to hold is $\mathbf{A} \succ \mathbf{0}$ and $s=c-\mathbf{b}^{H} \mathbf{A}^{-1} \mathbf{b} \geq 0$. For the LMI in (2.23c), $\mathbf{A}=\mathbf{Q}_{k}+\lambda_{k} \mathbf{I}_{N_{t}}+\mu_{k} \mathbf{I}_{N_{t}}$. If we let $\rho_{k}(\cdot)$ denote the $k^{t h}$ eigenvalue of a symmetric matrix, then $\rho_{k}(\mathbf{A})=\rho_{k}\left(\mathbf{Q}_{k}\right)+\lambda_{k}+\mu_{k}$, where the power constraints ensures that $\rho_{k}\left(\mathbf{Q}_{k}\right)$ is bounded (both above and below). With $\rho_{k}\left(\mathbf{Q}_{k}\right)$ being bounded and $\lambda_{k}=r_{k}^{\star} / \varepsilon_{0}^{2}$, since (2.50) implicitly maximizes the smallest of the $r_{k}$ 's, for sufficiently small $\varepsilon_{0}$, the optimal solution for (2.50) will be such that there is a large range of values 
for $\mu_{k}$ such that $\mathbf{A} \succ \mathbf{0}$. The Schur complement of $\mathbf{A}$ in the LMI in (2.23c) is $s=\left(\mathbf{h}_{q_{k}}^{H} \mathbf{Q}_{k} \mathbf{h}_{q_{k}}-\sigma_{k}^{2} / \alpha_{k}-r_{k}\right)-\mathbf{y}^{H} \mathbf{A}^{-1} \mathbf{y}$, where $\mathbf{y}=\mathbf{Q}_{k} \mathbf{h}_{q_{k}}+\mu_{k} \mathbf{h}_{q_{k}}$. Since the solution to (2.50) guarantees that $\mathbf{h}_{q_{k}}^{H} \mathbf{Q}_{k} \mathbf{h}_{q_{k}}-\sigma_{k}^{2} / \alpha_{k}-r_{k} \geq \delta>0$, we need to show that for sufficiently small $\varepsilon_{0}$, there exist a $\mu_{k}$ such that $\mathbf{y}^{H} \mathbf{A}^{-1} \mathbf{y} \leq \delta$, while $\mathbf{A}$ remains positive definite. To do so, we observe that when $\lambda_{k}$ is large $\mathbf{A}^{-1}$ will have small eigenvalues, which will ensure that $\mathbf{y}^{H} \mathbf{A}^{-1} \mathbf{y}$ is small since $\mathbf{y}$ is bounded. To show that explicitly, we observe that if $\mathbf{Q}_{k}=\mathbf{V}_{k} \operatorname{Diag}\left(\rho_{k}\left(\mathbf{Q}_{k}\right)\right) \mathbf{V}_{k}^{H}$ denotes the eigen decomposition of $\mathbf{Q}_{k}$, we can rewrite $\mathbf{y}^{H} \mathbf{A}^{-1} \mathbf{y}=\mathbf{h}_{q_{k}}^{H} \mathbf{V}_{k} \operatorname{Diag}\left(\frac{\left(\rho_{k}\left(\mathbf{Q}_{k}\right)+\mu_{k}\right)^{2}}{\rho_{k}\left(\mathbf{Q}_{k}\right)+\mu_{k}+\lambda_{k}}\right) \mathbf{V}_{k}^{H} \mathbf{h}_{q_{k}}$. The above arguments establish that for any given $\delta$, there is a sufficiently small $\varepsilon_{0}$ for which the solution to $(2.50)$ can be augmented by a choice for $\left\{\mu_{k}\right\}$ that results in a feasible point for (2.23). Since (2.23) and (2.26) have the same objective, and the matrices $\left\{\mathbf{W}_{k}\right\}$ in both problems are bounded, that establishes that as $\varepsilon_{0} \rightarrow 0$, any optimal set $\left\{\mathbf{W}_{k}\right\}$ for (2.26) approaches an optimal set $\left\{\mathbf{W}_{k}\right\}$ for (2.23).

\section{D Appendix: Rank one proof}

Analysis of the problem in (2.26) reveals that at optimality (2.26c) holds with equality. Indeed if that were not the case, then we could choose a larger $r_{k}$ and maintain feasibility. A larger $r_{k}$ would not decrease the objective value. Furthermore, at optimality all $r_{k}$ s are equal. To show that we observe that if, at optimality, $r_{1}$ was smaller than the other $r_{k} \mathrm{~s}$ then we could decrease the power of any of $\mathbf{W}_{k}, k \geq 2$, which would retain feasibility and yet allow for a larger value for $r_{1}$. That would contradict the assumed optimality.

To establish that (2.27) has an optimal solution that has rank one, we first assume that we have solved (2.27) for the power constraint $P$ and have obtained the optimal 
value $r_{\max }$. Now let us consider the problem,

$$
\begin{aligned}
P^{\star}=\min _{\mathbf{W}_{k}} & \sum_{k=1}^{K} \operatorname{tr}\left(\mathbf{W}_{k}\right), \\
& \mathbf{h}_{q_{k}}^{H} \mathbf{Q}_{k} \mathbf{h}_{q_{k}}-\sigma_{k}^{2} / \alpha_{k}-r_{\max } \geq 0, \\
& \mathbf{W}_{k} \succeq \mathbf{0} .
\end{aligned}
$$

The optimal value of this problem is $P^{\star}=P$. If $P^{\star}<P$, that would contradict $r_{\max }$ being the optimal value of (2.27), and if $P^{\star}>P$ that would contradict $r_{\max }$ being feasible for (2.27). Since $\sigma_{k}^{2} / \alpha_{k}+r_{\max }$ is a constant, the problem in (2.51) is precisely in the form of minimizing the transmitted power subject to SINR constraints in the presence of perfect CSI, but with a mismatched noise variance. That problem is known to have an optimal solution that has rank one (Bengtsson and Ottersten, 2001). 


\title{
Chapter 3
}

\section{Offset-Based Beamforming: A New Approach to Robust Downlink Transmission}

\begin{abstract}
The design of a set of beamformers for the multi-user multiple-input single-output (MISO) downlink so that the receivers are provided with prespecified levels of qualityof-service (QoS) can be quite challenging when the channel state information is not perfectly known at the base station. The constraint of having the SINR meet or exceed a given threshold with high probability is intractable in general, which results in problems that are fundamentally hard to solve. In this paper, we will develop a highquality approximation of the SINR outage constraint that enables us to formulate the precoder design problem as a convex optimization problem that can be efficiently solved. For systems in which the uncertainty size is small, a further approximation yields algorithms based on iterative evaluations of closed-form expressions that have
\end{abstract}


substantially lower computational cost. Since finding the beamforming directions incurs most of the computational load of these algorithms, analogous power loading algorithms for predefined beamforming directions are developed and their performance is shown to be close to optimal. When the system contains a large number of antennas, the proposed power loading can be obtained at a computational cost that grows only linearly in the number of antennas. The proposed power loading algorithm provides an explicit relationship between the outage probability required and the power consumed, which allows us to precisely control the power consumption, and automatically identifies users who are consuming most of the power resources .

\subsection{Introduction}

The directional signalling capabilities of base stations (BSs) that have multiple transmit antennas enable a variety of signalling techniques (Alodeh et al., 2017) for simultaneously transmitting independent messages to multiple single-antenna receivers, including dirty paper coding (Weingarten et al., 2006), vector perturbation precoding (Hochwald et al., 2005), lattice reduction precoding (Windpassinger et al., 2004a), Tomlinson-Harashima precoding (Windpassinger et al., 2004b), rate splitting (Joudeh and Clerckx, 2016), per-symbol beamforming (Alodeh et al., 2015), and conventional linear beamforming (Gesbert et al., 2007). Of these signalling techniques, conventional linear beamforming has the simplest implementation and will be the focus of this paper. In particular, we will consider scenarios in which the users that have been scheduled for transmission specify the quality-of-service (QoS) that they expect to receive. In that setting, the BS designs the set of beamformers to ensure that the signal-to-interference-and-noise ratio (SINR) at each receiver meets the target 
level that is implicitly specified by that user's QoS requirements. When the BS has perfect knowledge of the channel to each user, the beamformers that minimize the total transmitted power required to achieve the SINR targets can be efficiently found (Rashid-Farrokhi et al., 1998a; Bengtsson and Ottersten, 2001; Schubert and Boche, 2004). However, in practice these channels are estimated and possibly predicted. In time division duplexing (TDD) systems the estimation is typically performed during the training phase on the uplink, whereas in frequency division duplexing (FDD) systems, each receiver estimates its channel and feeds back a quantized version of that estimate to the BS. Since the BS has only estimates of the users' channels, it can only estimate the receivers' SINRs. Those estimates are, quite naturally, uncertain and hence there is a possibility that a design performed using the estimated channels will fail to meet the SINR targets when the beamformers are implemented.

A prominent approach to designing a precoder that can control the consequent outage is to postulate a model for the uncertainty in the channel estimates and to seek designs that control the outage probability under that uncertainty model. In some cases the approach involves jointly designing the beamforming directions and the power allocated to these directions (e.g., Kandukuri and Boyd, 2002; Shenouda and Davidson, 2008b; Wang et al., 2014; Medra et al., 2016), while in other cases the beamforming directions are designed based on the channel estimates only, and the uncertainty model is incorporated into the design of the power loading (e.g., Vucic and Boche, 2009a; Sohrabi and Davidson, 2016; Medra and Davidson, 2015a; PascualIserte et al., 2006). Unfortunately, in most settings the outage constraint has proven to be intractable (except for the case in Sohrabi and Davidson, 2016), and hence the goal has been to develop computationally efficient algorithms that can manage the 
outage probability. One possible strategy for doing so is to seek "safe" or conservative approximations of the robust optimization problem. When such approximations are feasible, their solution is guaranteed to satisfy the constraints of the original problem (Ben-Tal et al., 2009), but these approximations can be quite conservative (e.g., Wang et al., 2014; Shenouda and Davidson, 2008b). An alternative strategy is to develop approximations of the outage constraint that typically provide good performance, but might not necessarily guarantee that their solution is feasible for the original problem (e.g., Medra et al., 2016; Kandukuri and Boyd, 2002). The approach taken in this paper falls into that class.

The development of the proposed offset-based approach begins with the rewriting of the SINR constraint as the non-negativity of a random variable. That random variable is a non-convex quadratic function of the uncertainties, in which the quadratic kernel is a quartic function of the beamformers. Then, we approximate the nonnegativity constraint on the random variable by the constraint that its mean is larger than a given multiple of its standard deviation. For the case of Gaussian channel uncertainty, the mean and standard deviation are quadratic and quartic function of the beamformers, respectively. That fact enables the application of semidefinite relaxation techniques to obtain a convex formulation of (a relaxed version of) the approximated problem. While that design technique has proven to be quite effective, the computational cost of solving the convex conic program with semidefinite constraints is significant. By making a further approximation that is suitable for systems with reasonably small uncertainties, we obtain a design formulation for which the KKT optimality conditions have a simpler structure. That simpler structure facilitates the 
development of an approximate solution method that only requires the iterative evaluation of closed-form expressions. Further approximations reveal a connection with the low-complexity technique developed by Medra et al. (2016).

An analysis of the computational cost of these precoder design techniques shows that it is the calculation of the beamforming directions that consumes most of the required computational resources, and that when these directions are defined inadvance, the computational load can be significantly reduced. Accordingly, we develop variants of our precoder design algorithms that perform power loading on the set of fixed beamforming directions. These algorithms have low computational costs, and provide performance that is close to that of the optimal power loading algorithm (Sohrabi and Davidson, 2016). Furthermore, for systems with a large number of antennas, (i.e., massive MIMO) in which the channel hardens, we develop a variant of our power loading algorithm that has a computational cost that grows only linearly with the number of antennas.

In practice, the BS has limited power available for transmission, and it is possible that the power required to serve the scheduled users with the required outage probabilities may exceed that limit. In some of these scenarios, some users suffer from a weak channel, or from having their channels closely aligned with those of other users. When that happens, such users consume most of the power transmitted by the BS, and this suggests opportunities to reschedule users. On the other hand, some users might be close to the BS and experiencing a relatively strong channel; a case that suggests opportunities for doing some sort of power saving. The proposed power loading algorithm provides an explicit relationship between the required outage probabilities and the consumed power, which allows us to address those issues. Using 
this explicit power-outage relationship we can reduce the required power when the resulting increases in the outage probabilities are tolerable, and we can identify users that consume excessive amounts of power.

\subsection{System model}

We consider a scenario in which a BS that has $N_{t}$ antennas communicates with $K$ single-antenna users over a narrow-band channel. In the linear beamforming transmission case, the transmitted signal can be written as $\mathbf{x}=\sum_{k=1}^{K} \mathbf{w}_{k} s_{k}$, where $s_{k}$ is the normalized data symbol intended for user $k$, and $\mathbf{w}_{k}$ is the associated beamformer vector. For later reference we let $\mathbf{u}_{k}=\mathbf{w}_{k} /\left\|\mathbf{w}_{k}\right\|$ denote the beamforming direction for user $k$, and let $\beta_{k}=\left\|\mathbf{w}_{k}\right\|^{2}$ denote the power allocated to that direction. Hence, $\mathbf{w}_{k}=\sqrt{\beta_{k}} \mathbf{u}_{k}$. The received signal at user $k$ is modelled as

$$
y_{k}=\mathbf{h}_{k}^{H} \mathbf{w}_{k} s_{k}+\sum_{j \neq k} \mathbf{h}_{k}^{H} \mathbf{w}_{j} s_{j}+n_{k}
$$

where $\mathbf{h}_{k}^{H}$ is the vector of complex channel gains between the antennas at the BS and user $k$, and $n_{k}$ is the additive zero-mean circular complex Gaussian noise at that user. Under this model, if we let $\sigma_{k}^{2}$ denote the noise variance, then the SINR at user $k$ is

$$
\operatorname{SINR}_{k}=\frac{\left|\mathbf{h}_{k}^{H} \mathbf{w}_{k}\right|^{2}}{\sum_{j \neq k}\left|\mathbf{h}_{k}^{H} \mathbf{w}_{j}\right|^{2}+\sigma_{k}^{2}}
$$

The design of a set of beamformers $\left\{\mathbf{w}_{k}\right\}_{k=1}^{K}$ so that the SINRs satisfy specified target values; i.e., $\operatorname{SINR}_{k} \geq \gamma_{k}$ requires the knowledge of the channel vectors $\left\{\mathbf{h}_{k}\right\}_{k=1}^{K}$. However, the BS has only estimates of $\left\{\mathbf{h}_{k}\right\}_{k=1}^{K}$, and its estimates of the SINRs at 
the receivers are uncertain. Accordingly, we will incorporate the channel uncertainty model into the design process. In particular, we will consider systems in which the uncertainty can be modelled using the simple additive model,

$$
\mathbf{h}_{k}=\mathbf{h}_{e_{k}}+\mathbf{e}_{k},
$$

where $\mathbf{h}_{e_{k}}$ is the BS's estimate of the channel to user $k$, and the uncertainty in that estimate is characterized by the distribution of the elements of $\mathbf{e}_{k}$. In this paper, we will focus on scenarios in which $\mathbf{e}_{k}$ can be modelled as a circular complex Gaussian random variable with mean $\mathbf{m}_{k}$ and covariance $\mathbf{C}_{k}$; i.e., $\mathbf{e}_{k} \sim \mathcal{C N}\left(\mathbf{m}_{k}, \mathbf{C}_{k}\right)$. One scenario in which is model ia applicable is that of a TDD scheme operating in a slow fading environment, in which the BS estimates the channel on the uplink using a linear estimator and exploits channel reciprocity. When the channel gains are uncorrelated and the BS employs the best linear unbiased estimator (BLUE), $\mathbf{e}_{k} \backsim \mathcal{C N}\left(0, \sigma_{e_{k}}^{2} \mathbf{I}\right)$, and we will pay particular attention to that case. (Robust beamforming schemes for uncertainty models tailored to the FDD case are developed by Medra et al. (2016).)

Now if we let $\delta_{k}$ denote the maximum tolerable outage probability for user $k$, the generic joint beamforming and power loading problem can be written as

$$
\begin{aligned}
\min _{\mathbf{w}_{k}} & \sum_{k=1}^{K} \mathbf{w}_{k}^{H} \mathbf{w}_{k} \\
\text { subject to } & \operatorname{Prob}\left(\operatorname{SINR}_{k} \geq \gamma_{k}\right) \geq 1-\delta_{k}, \quad \forall k .
\end{aligned}
$$

This problem is hard to solve due to the intractable probabilistic outage constraint in (3.4b) even when the uncertainty is Gaussian (Kandukuri and Boyd, 2002; Shenouda and Davidson, 2008b; Wang et al., 2014). In order to resolve that intractability, a 
variety of approximations of the problem in (3.4) by problems that are tractable have been proposed (Kandukuri and Boyd, 2002; Shenouda and Davidson, 2008b; Wang et al., 2014; Medra et al., 2016). However, many of those alternate problems are based on conservative approximations, in the sense that the feasible set of beamformers for the safe approximation is significantly smaller than the actual feasible set in (3.4b).

\subsection{Principles of Offset-based approach}

The derivation of the proposed approximation of the outage probability begins by rewriting $\operatorname{SINR}_{k} \geq \gamma_{k}$ as $\mathbf{h}_{k}^{H} \mathbf{Q}_{k} \mathbf{h}_{k}-\sigma_{k}^{2} \geq 0$, where

$$
\begin{aligned}
\mathbf{Q}_{k} & =\mathbf{w}_{k} \mathbf{w}_{k}^{H} / \gamma_{k}-\sum_{j \neq k} \mathbf{w}_{j} \mathbf{w}_{j}^{H} \\
& =\beta_{k} \mathbf{u}_{k} \mathbf{u}_{k}^{H} / \gamma_{k}-\sum_{j \neq k} \beta_{j} \mathbf{u}_{j} \mathbf{u}_{j}^{H} .
\end{aligned}
$$

That is, the probability that $\operatorname{SINR}_{k} \geq \gamma_{k}$ is the same as the probability that the term $\mathbf{h}_{k}^{H} \mathbf{Q}_{k} \mathbf{h}_{k}-\sigma_{k}^{2}$ is non-negative. Under the additive uncertainty model in (3.3), we observe that $\mathbf{h}_{k}^{H} \mathbf{Q}_{k} \mathbf{h}_{k}-\sigma_{k}^{2}$ is an indefinite quadratic function of the uncertainty, $\mathbf{e}_{k}$. In particular, we can formulate the SINR constraint as follows

$$
f_{k}\left(\mathbf{e}_{k}\right)=\mathbf{h}_{e_{k}}^{H} \mathbf{Q}_{k} \mathbf{h}_{e_{k}}+2 \operatorname{Re}\left(\mathbf{e}_{k}^{H} \mathbf{Q}_{k} \mathbf{h}_{e_{k}}\right)+\mathbf{e}_{k}^{H} \mathbf{Q}_{k} \mathbf{e}_{k}-\sigma_{k}^{2} \geq 0 .
$$

The key observation that underlies the offset approximation is that for uncertainties $\mathbf{e}_{k}$ that are reasonably concentrated, if we design the beamforming vectors so that the mean value of $f_{k}\left(\mathbf{e}_{k}\right)$, denoted by $\mu_{f_{k}}$, is a significant multiple of its standard deviation, denoted by $\sigma_{f_{k}}$, then that user will achieve a low outage probability. If we let $r_{k}$ denote that multiple for the $k$ th user, then the resulting approximation of the 
SINR constraint, $\operatorname{Prob}\left(\operatorname{SINR}_{k} \geq \gamma_{k}\right) \geq 1-\delta_{k}$, can be written as

$$
\mu_{f_{k}} \geq r_{k} \sigma_{f_{k}}
$$

In order to develop an intuitive rationale for that approximation for the outage probability, we observe that when $\mathbf{e}_{k}$ in (3.3) is Gaussian, $f_{k}\left(\mathbf{e}_{k}\right)$ has a generalized chi-square distribution (Al-Naffouri et al., 2016). We also observe that the term that complicates the calculation of the relevant tail probability (i.e., $\operatorname{Prob}\left(f_{k}\left(\mathbf{e}_{k}\right)<0\right)$ ) is the indefinite quadratic term $\mathbf{e}_{k}^{H} \mathbf{Q}_{k} \mathbf{e}_{k}$ in (3.6). To have reasonable outage performance, the norm of the channel uncertainty $\mathbf{e}_{k}$ in (3.3) should be relatively small compared to the norm of the channel (cf. Jindal, 2006). In that case, the constant and linear terms in (3.6) will tend to dominate the quadratic term. Furthermore, the distribution of $\mathbf{e}_{k}^{H} \mathbf{Q}_{k} \mathbf{e}_{k}$ is "bell shaped" since $\mathbf{Q}_{k}$ generically has one positive and $K-1$ negative eigenvalues. Now if we approximate the quadratic term $\mathbf{e}_{k}^{H} \mathbf{Q}_{k} \mathbf{e}_{k}$ by a Gaussian term of the same mean and variance, then the distribution of $f_{k}\left(\mathbf{e}_{k}\right)$ becomes Gaussian and the constraint in (3.7) provides precise control over the tail probability. In other words, the constraint in (3.7) provides precise control of the tail probability of the Gaussian approximation of $f_{k}\left(\mathbf{e}_{k}\right)$. These insights, and the guidance that they provide on the choice of $r_{k}$, are discussed more formally in Appendix 3.A.

To be able to use the offset approximation in (3.7) in a low-complexity design algorithm, we need to obtain expressions for $\mu_{f_{k}}$ and $\sigma_{f_{k}}$ in terms of the design 
variables $\mathbf{w}_{k}=\sqrt{\beta_{k}} \mathbf{u}_{k}$. As shown in Appendix 3.B, when $\mathbf{e}_{k} \backsim \mathcal{C N}\left(\mathbf{m}_{k}, \mathbf{C}_{k}\right)$

$$
\begin{aligned}
\mu_{f_{k}} & =\mathbb{E}\left\{f_{k}\left(\mathbf{e}_{k}\right)\right\} \\
& =\left(\mathbf{h}_{e_{k}}+\mathbf{m}_{k}\right)^{H} \mathbf{Q}_{k}\left(\mathbf{h}_{e_{k}}+\mathbf{m}_{k}\right)-\sigma_{k}^{2}+\mathbf{w}_{k}^{H} \mathbf{C}_{k} \mathbf{w}_{k} / \gamma_{k}-\sum_{j \neq k} \mathbf{w}_{j}^{H} \mathbf{C}_{k} \mathbf{w}_{j}^{H}, \\
\sigma_{f_{k}}^{2} & =\operatorname{var}\left\{f_{k}\left(\mathbf{e}_{k}\right)\right\} \\
& =2\left(\mathbf{h}_{e_{k}}+\mathbf{m}_{k}\right)^{H} \mathbf{C}_{k}^{1 / 2} \mathbf{Q}_{k}^{2} \mathbf{C}_{k}^{1 / 2}\left(\mathbf{h}_{e_{k}}+\mathbf{m}_{k}\right)+\operatorname{tr}\left(\mathbf{C}_{k}^{1 / 2} \mathbf{Q}_{k} \mathbf{C}_{k}^{1 / 2}\right)^{2} .
\end{aligned}
$$

An important observation is that $\mu_{f_{k}}$ is a non-convex quadratic function of the beamformers $\left\{\mathbf{w}_{k}\right\}_{k=1}^{K}$, but for fixed beamforming directions $\left\{\mathbf{u}_{k}\right\}_{k=1}^{K}$ it is a linear function of the power loading $\left\{\beta_{k}\right\}_{k=1}^{K}$. The variance $\sigma_{f_{k}}^{2}$ is a quartic function of the beamformers, and for fixed directions is a non-convex quadratic function of the power loading. In scenarios in which the model $\mathbf{e}_{k} \backsim \mathcal{C N}\left(0, \sigma_{e_{k}}^{2} \mathbf{I}\right)$ is appropriate, these expressions simplify to

$$
\begin{aligned}
& \mu_{f_{k}}=\mathbf{h}_{e_{k}}^{H} \mathbf{Q}_{k} \mathbf{h}_{e_{k}}-\sigma_{k}^{2}+\sigma_{e_{k}}^{2}\left(\beta_{k} / \gamma_{k}-\sum_{j \neq k} \beta_{j}\right) . \\
& \sigma_{f_{k}}^{2}=2 \sigma_{e_{k}}^{2} \mathbf{h}_{e_{k}}^{H} \mathbf{Q}_{k}^{2} \mathbf{h}_{e_{k}}+\sigma_{e_{k}}^{4} \operatorname{tr}\left(\mathbf{Q}_{k}^{2}\right) .
\end{aligned}
$$

We will focus on this simplified case in the following sections.

\subsection{Offset-based robust beamforming}

As discussed above, the robust beamforming problem in (3.4) is fundamentally hard to solve due to the intractability of the probabilistic SINR outage constraint in (3.4b). If we were to replace that constraint with its offset approximation, $\mu_{f_{k}} \geq r_{k} \sigma_{f_{k}}$, then 
the problem in (3.4) can be closely approximated by

$$
\begin{array}{cl}
\min _{\mathbf{w}_{k}} & \sum_{k=1}^{K} \mathbf{w}_{k}^{H} \mathbf{w}_{k} \\
\text { subject to } & \mu_{f_{k}} \geq r_{k} \sigma_{f_{k}}, \quad \forall k .
\end{array}
$$

Some insights into the behaviour of solutions to (3.10) can be obtained by observing that when the values of $r_{k}$ are chosen to be the same, the beamforming vectors are designed so that users with a large SINR variance are provided with a larger SINR mean. To do so, those users with a lower SINR variance are not provided with as large mean SINR as they do not need the same protection against the uncertainty.

To develop an algorithm to obtain good solutions to (3.10), we observe that in (3.10b) we have the term $\mu_{f_{k}}$ which is quadratic in $\mathbf{w}_{k}$, and we also have the term $\sigma_{f_{k}}^{2}=2 \sigma_{e_{k}}^{2} \mathbf{h}_{e_{k}}^{H} \mathbf{Q}_{k}^{2} \mathbf{h}_{e_{k}}+\sigma_{e_{k}}^{4} \operatorname{tr}\left(\mathbf{Q}_{k}^{2}\right)$, which includes the square of the matrix $\mathbf{Q}_{k}$ and, accordingly, is quartic in $\mathbf{w}_{k}$. If we make the substitution $\mathbf{W}_{k}=\mathbf{w}_{k} \mathbf{w}_{k}^{H}$, then the functions in (3.10b) become linear and quadratic functions of $\mathbf{W}_{k}$ and the objective becomes linear. As such, the remaining difficulty in the reformulation of the problem is the set of rank-one constraints on $\mathbf{W}_{k}$. If we relax those constraints we obtain the 
following semidefinite relaxation of the problem in (3.10)

$$
\begin{aligned}
\min _{\mathbf{W}_{k}, d_{1 k}, d_{2 k}} & \operatorname{tr}\left(\sum_{k=1}^{K} \mathbf{W}_{k}\right) \\
\text { s.t. } & \mathbf{h}_{e_{k}}^{H} \mathbf{Q}_{k} \mathbf{h}_{e_{k}}-\sigma_{k}^{2}+\sigma_{e_{k}}^{2} \operatorname{tr}\left(\mathbf{W}_{k}\right) / \gamma_{k}-\sigma_{e_{k}}^{2} \operatorname{tr}\left(\sum_{j \neq k} \mathbf{W}_{j}\right) \\
& \geq r_{k}\left\|\left[d_{1 k} d_{2 k}\right]\right\|, \\
& d_{1 k} \geq \sqrt{2} \sigma_{e_{k}}\left\|\mathbf{h}_{e_{k}}^{H} \mathbf{Q}_{k}\right\|, \\
& d_{2 k} \geq \sigma_{e_{k}}^{2}\left\|\mathbf{Q}_{k}\right\|_{F}, \\
& \mathbf{W}_{k} \succeq \mathbf{0}, \quad \forall k,
\end{aligned}
$$

where $\|\cdot\|_{F}$ represents the Frobenius norm of the matrix. In this formulation, each SINR constraint in (3.10b) is replaced by three second order cone constraints (SOCs). Thus, the problem in (3.11) is a convex conic optimization problem and can be efficiently solved using interior point methods. Two refined implementations of those methods are easily accessible through the MatLaB-based CVX tool (Grant et al., 2008). In our numerical experience, the rank of the optimal $\mathbf{W}_{k}$ 's in (3.11) has always been one. When that occurs, the semidefinite relaxation is tight and the optimal beamformer vectors $\mathbf{w}_{k}$ can be directly obtained from the optimal matrices $\mathbf{W}_{k}$. This

phenomenon has been established in related beamforming problems (Bengtsson and Ottersten, 2001; Song et al., 2012; Ma et al., 2017), and has been observed numerically in a number of other downlink beamforming problems (e.g., Wang et al., 2014).

\subsubsection{Low-complexity precoding algorithm}

Although the problem in (3.11) is convex, it contains $3 K$ SOC constraints, plus the $K$ semidefinite constraints. As a result, solving (3.11) incurs a significant computational 
load even for a moderate number of antennas. In this section, we will first show how a mild approximation of the problem in (3.11) leads to an optimization problem with only $K \mathrm{SOC}$ constraints. We will then use insights from the KKT conditions of that problem to show that it can be approximately solved using the iterative evaluation of a sequence of closed-form expressions.

The approximation is based on the observation, made above, that in practical downlink systems the uncertainty in the channel estimates must be small in order for the system to support reasonable rates (Jindal, 2006). In such scenarios, the term in (3.10b) containing $\sigma_{e_{k}}^{4}$ will typically be significantly smaller than the other term. Accordingly, $\sigma_{f_{k}}^{2} \approx 2 \sigma_{e_{k}}^{2} \mathbf{h}_{e_{k}}^{H} \mathbf{Q}_{k}^{2} \mathbf{h}_{e_{k}}$ is a reasonable approximation. Applying this approximation in the context of the problem in (3.10) we obtain the following approximation of (3.10b)

$$
\mathbf{h}_{e_{k}}^{H} \mathbf{Q}_{k} \mathbf{h}_{e_{k}}-\sigma_{k}^{2}+\sigma_{e_{k}}^{2} \mathbf{w}_{k}^{H} \mathbf{w}_{k} / \gamma_{k}-\sigma_{e_{k}}^{2} \sum_{j \neq k} \mathbf{w}_{j}^{H} \mathbf{w}_{j} \geq r_{k} \sqrt{2} \sigma_{e_{k}}\left\|\mathbf{h}_{e_{k}}^{H} \mathbf{Q}_{k}\right\|
$$

The semidefinite relaxation of the resulting approximation of the problem in (3.10) can be written as

$$
\begin{aligned}
\min _{\mathbf{W}_{k}, \mathbf{d}_{k}} & \operatorname{tr}\left(\sum_{k=1}^{K} \mathbf{W}_{k}\right) \\
\text { s.t. } & \mathbf{h}_{e_{k}}^{H} \mathbf{Q}_{k} \mathbf{h}_{e_{k}}-\sigma_{k}^{2}+\sigma_{e_{k}}^{2} \operatorname{tr}\left(\mathbf{W}_{k}\right) / \gamma_{k}-\sigma_{e_{k}}^{2} \operatorname{tr}\left(\sum_{j \neq k} \mathbf{W}_{k}\right) \\
& \geq\left\|\mathbf{d}_{k}\right\|, \\
& \mathbf{d}_{k}=r_{k} \sqrt{2} \sigma_{e_{k}} \mathbf{Q}_{k} \mathbf{h}_{e_{k}}, \\
& \mathbf{W}_{k} \succeq \mathbf{0}, \quad \forall i .
\end{aligned}
$$


We note that the problem in (3.13) is over parameterized (the vectors $\mathbf{d}_{k}$ are not needed), but this over parameterization will simplify the following analysis.

The problem in (3.13) is another convex conic program, but it has significantly fewer constraints than that in (3.11); there are $K$ SOC constraints rather than the $3 K$ in (3.11). While it can be solved with less computational effort than (3.11), the presence of the semidefinite constraints means that considerable effort is still required. To derive a more efficient algorithm, we examine the Lagrangian of (3.13), assuming that the matrices $\mathbf{W}_{k}$ are of rank one. If we let $\nu_{k}$ denote the dual variable for the constraint in (3.13b), and $\boldsymbol{\psi}_{f_{k}}$ denote the vector of dual variables for the equality constraint in (3.13c), the Lagrangian can be written as

$$
\begin{aligned}
\mathcal{L}\left(\mathbf{w}_{k}, \mathbf{d}_{k}, \nu_{k}, \boldsymbol{\psi}_{f_{k}}\right) & =\sum_{k=1}^{K} \mathbf{w}_{k}^{H} \mathbf{w}_{k}-\sum_{k=1}^{K} \nu_{k}\left(\mathbf{h}_{e_{k}}^{H} \mathbf{Q}_{k} \mathbf{h}_{e_{k}}-\sigma_{k}^{2}+\sigma_{e_{k}}^{2} \mathbf{w}_{k}^{H} \mathbf{w}_{k} / \gamma_{k}\right. \\
& \left.-\sigma_{e_{k}}^{2} \sum_{j \neq k} \mathbf{w}_{j}^{H} \mathbf{w}_{j}-\left\|\mathbf{d}_{k}\right\|\right)-\sum_{k=1}^{K} \boldsymbol{\psi}_{f_{k}}^{H}\left(\mathbf{d}_{k}-r_{k} \sqrt{2} \sigma_{e_{k}} \mathbf{Q}_{k} \mathbf{h}_{e_{k}}\right) .
\end{aligned}
$$

From the KKT conditions of the problem in (3.13), we can deduce that

$$
\begin{aligned}
\mathbf{w}_{k}=\left(\frac{\nu_{k}}{\gamma_{k}} \mathbf{h}_{e_{k}} \mathbf{h}_{e_{k}}^{H}-\sum_{j \neq k} \nu_{j} \mathbf{h}_{e_{j}} \mathbf{h}_{e_{j}}^{H}+\frac{\nu_{k} \sigma_{e_{k}}^{2}}{\gamma_{k}} \mathbf{I}-\sum_{j \neq k} \nu_{j} \sigma_{e_{k}}^{2} \mathbf{I}\right. \\
\left.\quad-\frac{r_{k} \sqrt{2} \sigma_{e_{k}}}{\gamma_{k}} \operatorname{Re}\left\{\boldsymbol{\psi}_{f_{k}} \mathbf{h}_{e_{k}}^{H}\right\}+\sum_{j \neq k} r_{j} \sqrt{2} \sigma_{e_{k}} \operatorname{Re}\left\{\boldsymbol{\psi}_{j} \mathbf{h}_{e_{j}}^{H}\right\}\right) \mathbf{w}_{k},
\end{aligned}
$$

which is an eigen equation for the direction $\mathbf{u}_{k}$. Using a similar approach to the perfect CSI case (Bjornson et al., 2014), we can rearrange this equation to obtain the 
following fixed-point equation for $\nu_{k}$,

$$
\begin{aligned}
\nu_{k}^{-1}=\mathbf{h}_{e_{k}}^{H}\left(\mathbf{I}+\sum_{j} \nu_{j} \mathbf{h}_{e_{j}} \mathbf{h}_{e_{j}}^{H}-\frac{\nu_{k} \sigma_{e_{k}}^{2}}{\gamma_{k}} \mathbf{I}+\sum_{j \neq k} \nu_{j} \sigma_{e_{k}}^{2} \mathbf{I}+\right. \\
\left.\quad \frac{r_{k} \sqrt{2} \sigma_{e_{k}}}{\gamma_{k}} \operatorname{Re}\left\{\boldsymbol{\psi}_{f_{k}} \mathbf{h}_{e_{k}}^{H}\right\}-\sum_{j \neq k} r_{j} \sqrt{2} \sigma_{e_{k}} \operatorname{Re}\left\{\boldsymbol{\psi}_{j} \mathbf{h}_{e_{j}}^{H}\right\}\right)^{-1} \mathbf{h}_{e_{k}}\left(1+\frac{1}{\gamma_{k}}\right) .
\end{aligned}
$$

The expressions in (3.15) and (3.16) share a similar structure to those obtained for the corresponding QoS problem in the case of perfect CSI at the BS (Bjornson et al., 2014), but the matrix components of each equation contain four additional terms that are dependent on the variance of the channel estimation error. To exploit this structure and obtain an efficient algorithm for good solutions to (3.13) we observe that if we were given $\left\{\boldsymbol{\psi}_{f_{k}}\right\}$, then we could solve the fixed-point equations in (3.16) for $\left\{\nu_{k}\right\}$, and then we could solve the eigen equations in (3.15) for the beamforming directions $\left\{\mathbf{u}_{k}\right\}$. The solution could then be completed by performing the appropriate power loading, which will be explained in the following section. Therefore, if we could find a reasonable approximation for the vectors $\boldsymbol{\psi}_{f_{k}}$, we would obtain an iterative closed-form solution. To do so, we observe that the variable $\mathbf{d}_{k}$ in (3.13c) appears in the Lagrangian in the term $\nu_{k}\left\|\mathbf{d}_{k}\right\|-\boldsymbol{\psi}_{f_{k}}^{H} \mathbf{d}_{k}$. Accordingly, from the stationarity component of the KKT conditions we have that $\left\|\boldsymbol{\psi}_{f_{k}}\right\|=\nu_{k}$ and that $\mathbf{d}_{k}$ and $\boldsymbol{\psi}_{f_{k}}$ are in the same direction; i.e., $\mathbf{d}_{k} /\left\|\mathbf{d}_{k}\right\|=\boldsymbol{\psi}_{f_{k}} /\left\|\boldsymbol{\psi}_{f_{k}}\right\|$. Accordingly, we can write

$$
\boldsymbol{\psi}_{f_{k}}=\nu_{k} \mathbf{d}_{k} /\left\|\mathbf{d}_{k}\right\|
$$

Since $\mathbf{d}_{k}=r_{k} \sqrt{2} \sigma_{e_{k}} \mathbf{Q}_{k} \mathbf{h}_{e_{k}}, \boldsymbol{\psi}_{f_{k}}$ explicitly depends on the beamforming directions, which have not yet been determined. However, we observe that if we substitute 
(3.17) into (3.16), the terms involving $\mathbf{d}_{k}$ are multiplied by the standard deviation of the error, $\sigma_{e_{k}}$. As we have already argued in the derivation of the approximations that lead to (3.11), $\sigma_{e_{k}}$ will be small in effective downlink beamforming schemes, and this suggests that reasonable initial approximations of the directions should yield a good approximation of $\left\{\nu_{k}\right\}$, and hence a good set of beamforming directions. We suggest the use of the zero-forcing (ZF) directions (Spencer et al., 2004) for the estimated channels, which we will denote by $\mathbf{u}_{z_{k}}$. When we use that initialization, the initial direction of $\mathbf{d}_{k}$ will be the same as $\mathbf{u}_{z_{k}}$, which allows us to rewrite the fixed-point equations in (3.16) as

$$
\begin{aligned}
\nu_{k}^{-1}=\mathbf{h}_{e_{k}}^{H}\left(\mathbf{I}+\sum_{j} \nu_{j} \mathbf{h}_{e_{j}} \mathbf{h}_{e_{j}}^{H}-\frac{\nu_{k} \sigma_{e_{k}}^{2}}{\gamma_{k}} \mathbf{I}+\sum_{j \neq k} \nu_{j} \sigma_{e_{k}}^{2} \mathbf{I}+\right. \\
\left.\quad \frac{r \sqrt{2} \sigma_{e_{k}} \nu_{k}}{\gamma_{k}} \operatorname{Re}\left\{\mathbf{u}_{z_{k}} \mathbf{h}_{e_{k}}^{H}\right\}-\sum_{j \neq k} r \sqrt{2} \sigma_{e_{k}} \nu_{j} \operatorname{Re}\left\{\mathbf{u}_{z_{j}} \mathbf{h}_{e_{j}}^{H}\right\}\right)^{-1} \mathbf{h}_{e_{k}}\left(1+\frac{1}{\gamma_{k}}\right) .
\end{aligned}
$$

The derivations outlined above are summarized in the sequence of closed-form operations in Alg. 1. While the initial approximation can be improved by using the beamformers obtained in step 4 to obtain a refined estimate of the direction of $\mathbf{d}_{k}$ and returning to step 2 of the algorithm, the simulation results in Section 3.6 suggest that the one-shot approach taken in Alg. 1 produces a solution whose performance is quite close to that of the first offset-based design formulation in (3.11). That suggests that in the scenarios that we have considered, the underlying approximations are working quite well. 


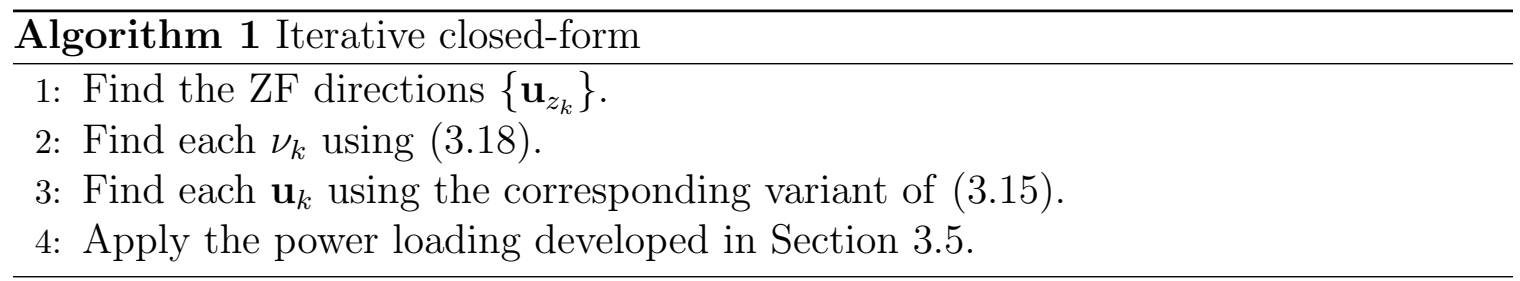

\subsubsection{Original constant-offset algorithm (Medra et al., 2016)}

As is apparent from the derivation in the previous section, one of the challenges that complicates the closed-form calculations is the quartic dependence of the variances $\sigma_{f_{k}}^{2}$ on the beamforming vectors $\mathbf{w}_{k}$. One way in which these complications can be reduced is to modify the offset approximation in (3.7) so that the mean, $\mu_{f_{k}}$, is constrained to be greater than a constant; i.e., the SINR constraint is replaced by

$$
\mu_{f_{k}} \geq r_{k} .
$$

If we make the approximation that the channel estimation errors are small enough that the third term on the right hand side of (3.9a) can be neglected, the semidefinite relaxation of the resulting approximation of (3.10) can be written as

$$
\begin{array}{ll}
\min _{\mathbf{W}_{k}} & \operatorname{tr}\left(\sum_{k=1}^{K} \mathbf{W}_{k}\right) \\
\text { s.t. } & \mathbf{h}_{e_{k}}^{H} \mathbf{Q}_{k} \mathbf{h}_{e_{k}}-\sigma_{k}^{2} \geq r_{k}, \\
& \mathbf{W}_{k} \succeq \mathbf{0}, \quad \forall k .
\end{array}
$$

Interestingly, this problem arose previously in the context of a low-complexity solution to the beamforming design in FDD systems, and the semidefinite relaxation was shown to be tight (Medra et al., 2016). 
The iterative closed-form solution to (3.19) has a similar structure to that in Alg. 1, but given the simpler structure of the problem, the Lagrange multipliers $\boldsymbol{\psi}_{f_{k}}$ disappear, and the expressions in (3.15) and (3.16) simplify to

$$
\begin{gathered}
\mathbf{w}_{k}=\left(\frac{\nu_{k}}{\gamma_{k}} \mathbf{h}_{e_{k}} \mathbf{h}_{e_{k}}^{H}-\sum_{j \neq k} \nu_{j} \mathbf{h}_{e_{j}} \mathbf{h}_{e_{j}}^{H}\right) \mathbf{w}_{k}, \\
\nu_{k}^{-1}=\mathbf{h}_{e_{k}}^{H}\left(\mathbf{I}+\sum_{j} \nu_{j} \mathbf{h}_{e_{j}} \mathbf{h}_{e_{j}}^{H}\right)^{-1} \mathbf{h}_{e_{k}}\left(1+\frac{1}{\gamma_{k}}\right) .
\end{gathered}
$$

After obtaining the beamforming directions from (3.21) and (3.20), the power loading in (Medra et al., 2016) is performed based on the fact that the constraints in (3.19b) should be satisfied with equality at optimality. (If this were not the case for constraint $k$, then the power allocated to $\mathbf{w}_{k}$ could be reduced in a way that will still satisfy

all the constraints and provide a lower objective value, contradicting the presumed optimality.) While doing so generates a solution to (3.19), significant performance gains can be obtained when the beamforming directions obtained from (3.20) are combined with the power loading algorithm presented in Section 3.5.

\subsubsection{Complexity analysis and further approximations}

The problems in (3.11) and (3.13) are convex optimization problems with secondorder cone and semidefinite constraints. General purpose interior point methods for such problems require $\mathcal{O}\left(K N_{t}^{6}\right)$ operation per iteration, which represents a significant computational load. In contrast, the key computational steps in the iterative closedform approximation, Alg. 1, are those in (3.15), (3.18) and the calculation of the ZF directions that are used in the initialization. The ZF directions can be obtained in $\mathcal{O}\left(N_{t}^{2} K\right)$ operations. The computational cost of solving (3.18) is dominated by 
the matrix inversion required for each user and hence it grows as $\mathcal{O}\left(N_{t}^{3} K\right)$. We can exploit the factorized matrix structure in (3.15) which allows for an efficient use of the power iteration method. The cost of that step grows as $\mathcal{O}\left(N_{t} K^{2}\right)$. We can see that it is the computation of the Lagrange multipliers (3.18) that requires most of the resources to compute the beamforming directions.

The original constant-offset algorithm (Medra et al., 2016) that was reviewed in Section 3.4.2 does not require an initial set of directions and the expression for $\nu_{k}$ is significantly simpler. In particular, the matrix to be inverted is the same for each user, which reduces the number of computations required to $\mathcal{O}\left(N_{t}^{3}\right)$. Furthermore, additional approximations can be applied to avoid the matrix inversion all together. When the channels are nearly orthogonal, as they tend to be in massive MISO channels that "harden" as the number of antennas increases (Hochwald et al., 2004), then if we let $\alpha_{k}=\left\|\mathbf{h}_{e_{k}}\right\|^{2}$, then we can write $\sum_{j} \nu_{j} \mathbf{h}_{e_{j}} \mathbf{h}_{e_{j}}^{H}$ as an eigen decomposition $\sum_{j} \nu_{j} \alpha_{j} \frac{\mathbf{h}_{e_{j}}}{\sqrt{\alpha_{j}}} \frac{\mathbf{h}_{e_{j}}^{H}}{\sqrt{\alpha_{j}}}$, and hence,

$$
\mathbf{h}_{e_{k}}^{H}\left(\mathbf{I}+\sum_{j} \nu_{j} \alpha_{j} \frac{\mathbf{h}_{e_{j}}}{\sqrt{\alpha_{j}}} \frac{\mathbf{h}_{e_{j}}^{H}}{\sqrt{\alpha_{j}}}\right)^{-1} \mathbf{h}_{e_{k}} \approx \frac{\alpha_{k}}{1+\nu_{k} \alpha_{k}} .
$$

Accordingly, we can approximate (3.21) by

$$
\nu_{k} \approx \gamma_{k} / \alpha_{k}
$$

To find the channel norms $\alpha_{k}=\left\|\mathbf{h}_{e_{k}}\right\|^{2}$ we need only $\mathcal{O}\left(N_{t}\right)$ operations. Hence, that approximation enables us to compute all $\nu_{k} \mathrm{~s}$ in only $\mathcal{O}\left(N_{t} K\right)$ operations. 


\subsection{Offset-based robust power Loading}

In this section, we will show how to apply the offset-based approach to the power loading problem that remains once the beamforming directions have been designed. Examples of choices for those directions include the maximum ratio transmission (MRT), zero-forcing (ZF), or regularized zero-forcing (RZF) directions, which are calculated from the estimated channels, or any of the directions generated by the previously described algorithms. Once the directions are chosen, we can rewrite the problem in (3.10) as

$$
\begin{array}{cl}
\min _{\beta_{k}} & \sum_{k=1}^{K} \beta_{k} \\
\text { subject to } & \mu_{f_{k}} \geq r_{k} \sigma_{f_{k}}, \quad \forall k,
\end{array}
$$

where for fixed directions $\left\{\mathbf{u}_{k}\right\}$ the expressions for $\mu_{f_{k}}$ and $\sigma_{f_{k}}$ in (3.9a) and (3.9b) simplify to

$$
\begin{aligned}
& \mu_{f_{k}}=\left|\mathbf{h}_{e_{k}}^{H} \mathbf{u}_{k}\right|^{2} \beta_{k} / \gamma_{k}-\sum_{j \neq k}\left|\mathbf{h}_{e_{k}}^{H} \mathbf{u}_{j}\right|^{2} \beta_{j}-\sigma_{k}^{2}+\sigma_{e_{k}}^{2}\left(\beta_{k} / \gamma_{k}-\sum_{j \neq k} \beta_{j}\right) . \\
& \sigma_{f_{k}}^{2}=2 \sigma_{e_{k}}^{2} \mathbf{h}_{e_{k}}^{H}\left(\beta_{k} \mathbf{u}_{k} \mathbf{u}_{k}^{H} / \gamma_{k}-\sum_{j \neq k} \beta_{j} \mathbf{u}_{j} \mathbf{u}_{j}^{H}\right)^{2} \mathbf{h}_{e_{k}}+\sigma_{e_{k}}^{4} \operatorname{tr}\left(\beta_{k} \mathbf{u}_{k} \mathbf{u}_{k}^{H} / \gamma_{k}-\sum_{j \neq k} \beta_{j} \mathbf{u}_{j} \mathbf{u}_{j}^{H}\right)^{2} .
\end{aligned}
$$

To develop an efficient algorithm for finding solutions to (3.22), we observe that at optimality the constraints in $(3.22 \mathrm{~b})$ hold with equality. If this were not the case for constraint $k$, then $\beta_{k}$ could be reduced in a way that still satisfies the constraints and yet provides a lower objective value, which would contradict the presumed optimality. 
To use that observation, we note that if the variances $\sigma_{f_{k}}^{2}$ are fixed, then the set of equations $\left\{\mu_{k}=r_{k} \sigma_{f_{k}}\right\}$ yields $K$ linear equations in the $K$ design variables $\left\{\beta_{k}\right\}_{k=1}^{K}$. If we define $\boldsymbol{\beta}=\left[\beta_{1}, \beta_{2}, \ldots, \beta_{K}\right]^{T}, \boldsymbol{\sigma}_{f}=\left[\sigma_{f_{1}}, \sigma_{f_{2}}, \ldots, \sigma_{f_{K}}\right]^{T}, \boldsymbol{\sigma}=\left[\sigma_{1}, \sigma_{2}, \ldots, \sigma_{K}\right]^{T}$, and the matrix A such that $[\mathbf{A}]_{i i}=\left|\mathbf{h}_{e_{i}}^{H} \mathbf{u}_{i}\right|^{2} / \gamma_{i}+\sigma_{e_{i}}^{2} / \gamma_{i}$, and $[\mathbf{A}]_{i j}=-\left|\mathbf{h}_{e_{i}}^{H} \mathbf{u}_{j}\right|^{2}-\sigma_{e_{i}}^{2}$, $\forall i \neq j$, then the set of linear equations can be written as

$$
\mathbf{A} \boldsymbol{\beta}=\boldsymbol{\sigma}^{2}+\boldsymbol{\sigma}_{f} \odot r_{k}
$$

in which $\odot$ represents element by element multiplication. Once the values of $\left\{\beta_{k}\right\}$ have been found, we can update the value of $\boldsymbol{\sigma}_{f}$ using (3.23b). That suggests the iterative linearization algorithm for solving (3.22) that is summarized in Alg. 2.

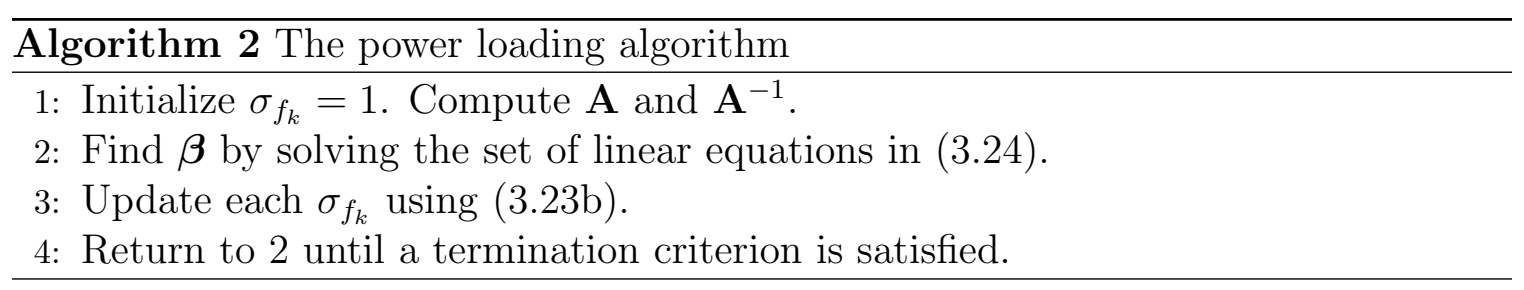

By observing the dependence of $\boldsymbol{\sigma}_{f}$ on $\boldsymbol{\beta}$ in (3.23b), Alg. 2 can be written in the form of a fixed point technique by writing $\boldsymbol{\beta}=\mathbf{A}^{-1} \boldsymbol{\sigma}^{2}+\mathbf{A}^{-1}\left(\boldsymbol{\sigma}_{f} \odot r_{k}\right)$. The eigenvalues of $\mathbf{A}^{-1}$ determine the convergence properties for these fixed-point equations. Since the matrix A typically has large diagonal values representing the signal powers, and lower values on the off-diagonal elements representing the interference powers, the eigen values of $\mathbf{A}^{-1}$ will typically be less than one. Our numerical experience not only confirms this observation, but also suggests that the number of iterations needed for near-optimal performance is very small. In terms of computational cost, the initialization step in Alg. 2 requires $\mathcal{O}\left(K^{2} N_{t}\right)$ operations to compute $\mathbf{A}$ and $\mathcal{O}\left(K^{3}\right)$ operations to compute $\mathbf{A}^{-1}$. In each iteration the computational cost for step 2 is 
$\mathcal{O}\left(K^{2}\right)$ operations, and the cost of step 3 is $\mathcal{O}\left(K N_{t}^{2}\right)$ operations.

\subsubsection{Simplifying the SINR variance calculation}

The above analysis shows that the only step in Alg. 2 whose computational cost grows faster than linearly in the number of antennas is the computation of $\sigma_{f_{k}}$. In massive MISO systems, the resulting computational load can be significant. To reduce the required computations, we observe that when the number of antennas is large and the channels are uncorrelated, the inner product between different channels will typically be relatively small. Since the beamforming directions will typically be closely aligned with the channel vectors, the inner product between different beamforming vectors will likely be small as well. This observation suggests removing the cross terms $\mathbf{u}_{j}^{H} \mathbf{u}_{k}, \forall j \neq k$ in (3.23b). That would yield the following approximations

$$
\begin{aligned}
\mathbf{h}_{e_{k}}^{H} \mathbf{Q}_{k} \mathbf{Q}_{k} \mathbf{h}_{e_{k}} & =\mathbf{h}_{e_{k}}^{H}\left(\beta_{k} \mathbf{u}_{k} \mathbf{u}_{k}^{H} / \gamma_{k}-\sum_{j \neq k} \beta_{j} \mathbf{u}_{j} \mathbf{u}_{j}^{H}\right)^{2} \mathbf{h}_{e_{k}} \\
& \approx\left|\mathbf{h}_{e_{k}}^{H} \mathbf{u}_{k}\right|^{2} \beta_{k}^{2} / \gamma_{k}^{2}+\sum_{j \neq k}\left|\mathbf{h}_{e_{k}}^{H} \mathbf{u}_{j}\right|^{2} \beta_{j}^{2},
\end{aligned}
$$

and

$$
\begin{aligned}
\operatorname{tr}\left(\mathbf{Q}_{k}^{2}\right) & =\operatorname{tr}\left(\beta_{k} \mathbf{u}_{k} \mathbf{u}_{k}^{H} / \gamma_{k}-\sum_{j \neq k} \beta_{j} \mathbf{u}_{j} \mathbf{u}_{j}^{H}\right)^{2} \\
& \approx \operatorname{tr}\left(\beta_{k}^{2} \mathbf{u}_{k} \mathbf{u}_{k}^{H} \mathbf{u}_{k} \mathbf{u}_{k}^{H} / \gamma_{k}^{2}+\sum_{j \neq k} \beta_{j}^{2} \mathbf{u}_{j} \mathbf{u}_{j}^{H} \mathbf{u}_{j} \mathbf{u}_{j}^{H}\right) \\
& =\beta_{k}^{2} / \gamma_{k}^{2}+\sum_{j \neq k} \beta_{j}^{2} .
\end{aligned}
$$

The numerical results presented in Section 3.6 indicate that these approximations result in designs that are very close in performance to those obtained from the original 
formulations, even when the number of antennas is quite small. Furthermore, since the terms $\left|\mathbf{h}_{e_{k}}^{H} \mathbf{u}_{j}\right|^{2}$ are already computed in the initialization step that constructs the matrix $\mathbf{A}$, these approximations reduce the computational cost of updating $\boldsymbol{\sigma}_{f}$ in step 3 of Alg. 2 from $\mathcal{O}\left(N_{t}^{2} K\right)$ to $\mathcal{O}\left(K^{2}\right)$.

\subsubsection{User rescheduling}

One of the fundamental characteristics of the original outage constrained beamformer design problem in (3.10) is that for a certain set of channel estimates the problem may be infeasible. That is, there may be no set of beamformers that can satisfy the outage constraints. Furthermore, even when the problem is feasible, the solution may be impractical in the sense that the minimum transmission power required to satisfy the outage constraints may exceed the capability of the BS. The approximations of the original formulation in (3.11), and (3.13) retain these characteristics, and the power loading problem in (3.22) retains them, too. Fortunately, as we now explain, for systems in which each user specifies the same value for $r$, the structure of a closely related power loading problem provides insights into which users should be rescheduled in order for the problem in (3.22) to be feasible, and for the solution of the problem to be within the capabilities of the BS. The auxiliary power loading problem that we will consider is that of maximizing a common offset coefficient subject to an explicit power constraint, namely

$$
\begin{array}{rl}
\max _{\beta_{k}, r} & r \\
\text { subject to } & \sum_{k=1}^{K} \beta_{k} \leq P_{t}, \\
& \mu_{k} \geq r \sigma_{f_{k}}, \quad \forall k,
\end{array}
$$


where $P_{t}$ denotes the maximum transmission power of the BS. This problem is always feasible whenever all the estimated channels are different. (The value of $r$ can be decreased until all components of (3.27c) can be satisfied using a power loading that satisfies (3.27b).) However, negative values and small positive values of $r$ correspond to cases with high probability of outage. The problem in (3.27) can be solved using an algorithm similar to that in Alg. 2. However, at the step analogous to step 2 of Alg. 2, we need an additional equation to determine the value for $r$. That equation arises from observing that the power constraint in $(3.27 \mathrm{~b})$ holds with equality at optimality, and hence, from (3.24) and (3.27b) we have that

$$
r=\frac{P_{t}-\mathbf{1}^{T} \mathbf{A}^{-1} \boldsymbol{\sigma}^{2}}{\mathbf{1}^{T} \mathbf{A}^{-1} \boldsymbol{\sigma}_{f}},
$$

where $\mathbf{1}$ is the vector with all elements equal to one. This equation clearly demonstrates the relationship between the power budget and the robustness. More importantly, it shows that these users that correspond to the largest elements of $\mathbf{A}^{-1} \boldsymbol{\sigma}^{2}$ are the ones that play the biggest role in constraining the extent of robustness that can be obtained. That suggests that if the optimal value of $r$ in (3.27) is not large enough to provide the desired robustness level, one or more of those users corresponding to large values of $\mathbf{A}^{-1} \boldsymbol{\sigma}^{2}$ should be rescheduled. (We note that the use of good user selection algorithms (e.g., Yoo and Goldsmith, 2006) prior to the design of the beamforming directions will reduce the need to reschedule users, but the inherent capability of the proposed power loading algorithms to perform rescheduling provides significant performance gains when the initial user selection is imperfect.)

Once the optimal value of the auxiliary problem in (3.27) exceeds the desired value for $r$, the power minimization problem in (3.22) can be solved. Since the distribution 
of $f_{k}\left(\mathbf{e}_{k}\right)$ is dominated by the Gaussian terms, values of $r$ in the range of 2 to 5 would be sufficient to obtain outage probabilities consistent with the expectations of contemporary applications.

\subsection{Simulation results}

In this section, we will provide three sets of numerical results. First, we will provide simulation results that show the validity of the offset-based algorithms and compare the performance of the algorithms presented here to that of algorithms that obtain robustness by ensuring that outage does not occur for uncertainties that lie in a given region. Specifically we will compare with the sphere bounding (SB) algorithm presented by Wang et al. (2014). Second, we will provide comparison between the performance of the power loading algorithms proposed in Section 3.5 and the optimal power loading algorithm by Sohrabi and Davidson (2016). In the third set of simulation results, we will demonstrate the performance gains that can be obtained by using the user rescheduling, and the power saving capabilities of the offset maximization framework.

For the simulation setups, we will we consider a downlink system in which a BS serves three single-antenna users. We will assume that the BS has four antennas, and the three users are randomly distributed within a radius of $3.2 \mathrm{~km}$. The large scale fading is described by a path-loss exponent of 3.52 and log-normal shadow fading with $8 \mathrm{~dB}$ standard deviation, and the small scale fading is modelled using the standard i.i.d. Rayleigh model. The channel estimation error is assumed to be zero-mean and Gaussian with covariance $\sigma_{e_{k}}^{2} \mathbf{I}$. The receiver noise level is $-90 \mathrm{dBm}$, and the SINR target is set to $6 \mathrm{~dB}$. A simple channel-strength user selection technique is employed, 
where users are served only if $100\left\|\mathbf{h}_{e_{k}}\right\|^{2} / \sigma_{k}^{2} \geq \gamma_{k}$, where we consider 100 here as the implicit total power constraint.

To plot the performance curves, we randomly generate a channel realization and provide a channel estimate and the corresponding error vector. Each of the algorithms is provided with the channel estimates and a robustness measure. The robustness measure for the algorithms provided in this paper is the value of the offset $r_{k}$. For the sphere bounding algorithm by Wang et al. (2014) it is the size of the zero-outage region, and for the power loading algorithm by Sohrabi and Davidson (2016) it is directly the outage probability. Each algorithm is used to design beamformers that should provide the specified robustness, and from those vectors we decide whether or not any user in the system with the actual channel realizations is in outage, and we calculate the corresponding transmission power. By repeating this experiment over thousands of channel realizations, we can plot the average power versus the outage probability for the different algorithms when these algorithms provide a feasible solution; a solution that satisfies the constraints using transmitted power that is less than 100. In fairness to all methods, the average is taken over those channel realizations for which all methods produce a feasible solution.

In Fig. 3.1, assuming $\sigma_{e_{k}}=0.1$, we plot the average total transmitted power versus the outage probability for the algorithms in (3.11), (3.13), Alg. 1, and that of the original offset maximization directions in Secion. 3.4.2 with the suggested power loading in Section 3.5. As benchmarks, we plot the performance of the SB algorithm (Wang et al., 2014), and that of the ZF when combined with the power loading in Section 3.5. In Fig. 3.2, we repeat the experiment for $\sigma_{e_{k}}=0.05$. We observe that the performance gap between the proposed algorithms becomes smaller when the error 


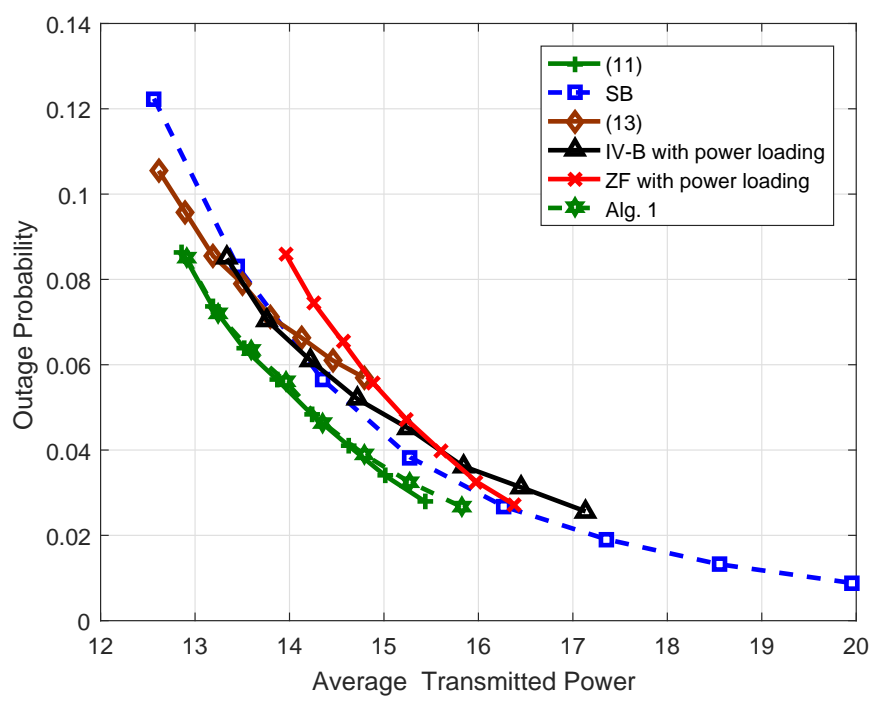

Figure 3.1: The average transmitted power against the outage probability for a system with 3 users, 4 BS antennas, $\gamma=6 \mathrm{~dB}$, and $\sigma_{e_{k}}=0.1$.

variance decreases, which justifies the validity of the approximations for small error size. We also note that the performance of Alg. 1 is very close to that of (3.11), and both of which are better than the SB-based algorithm. The performance of the ZF algorithm when combined with the proposed power loading algorithm in Section 3.5 depends on the uncertainty size, where better performance results are shown when the uncertainty size is larger. The combination of the original constant-offset directions in Section 3.4.2 with the suggested power loading in Section 3.5 seems to provide worse performance compared to other offset-based approaches. However, the decoupling of the beamforming directions design and the power loading can provide significant improvements in the performance as explained in Section 3.5. The performance gains come from the ability to control the power precisely, especially when the problem is infeasible. 


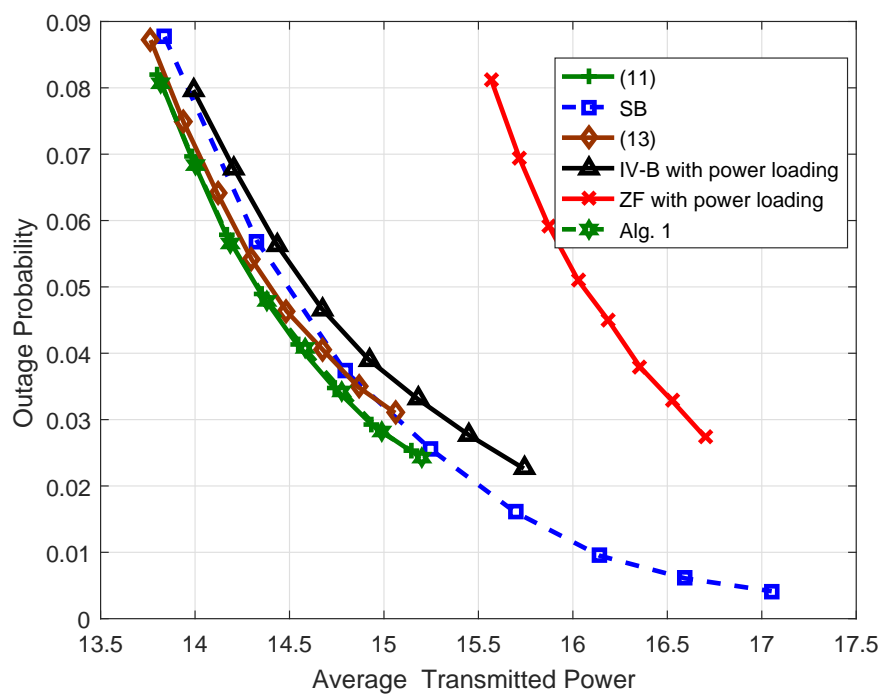

Figure 3.2: The average transmitted power against the outage probability for a system with 3 users, $4 \mathrm{BS}$ antennas, $\gamma=6 \mathrm{~dB}$, and $\sigma_{e_{k}}=0.05$.

The second set of simulation results provides the performance gap between the proposed power loading algorithm in Section 3.5, and the power loading algorithm by Sohrabi and Davidson (2016). In Fig. 3.3, we plot the average outage probability versus the average transmitted power for the power loading algorithm by Sohrabi and Davidson (2016), and the power loading in Alg. 2. While the algorithm by Sohrabi and Davidson (2016) is optimal in terms of the required power to achieve certain outage probability over the Gaussian error distribution, the proposed algorithm provides better average outage probability over time. This performance is achieved while having iterations no more than 5 .

To assess the performance gains that result from the power control capabilities of the proposed power loading algorithm, we plot the outage probability of the problem in (3.27) with the offset maximization directions in Section 3.4.2 versus the number 


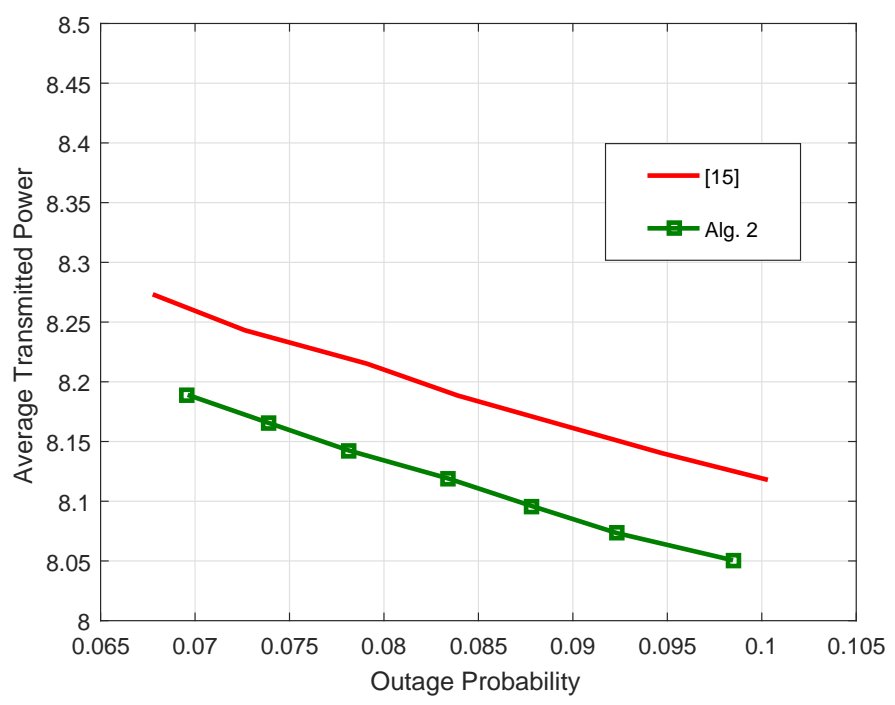

Figure 3.3: The average transmitted power against the outage probability for a system with 3 users, 4 BS antennas, $\gamma=6 \mathrm{~dB}$, and $\sigma_{e_{k}}=0.1$. Here [15] referes to (Sohrabi and Davidson, 2016).

of antennas. In this case, we set the total power constraint $P_{t}=1$, and the number of users to six. We plot the offset maximization when the approximations for obtaining the directions in Section 3.4.3, and those for obtaining the power loading in Section 3.5.1 are applied. We also plot the performance of the proposed user rescheduling scheme (Alg. Sect. 3.5.2 (a)) and the user rescheduling when combined with the power saving (Alg. Sect. 3.5.2 (b)). We observe that the proposed approximations provide almost the same outage performance over the whole range of antenna numbers. We also observe that the user rescheduling technique greatly enhances the outage performance, especially when the number of antennas is relatively low, which can be translated to a greater probability of the channels not being sufficiently orthogonal. (In this example, we apply user rescheduling whenever the resulting offset $\mathrm{r}$ in (3.27) is smaller than two, and the rescheduled user(s) are considered in outage.) 


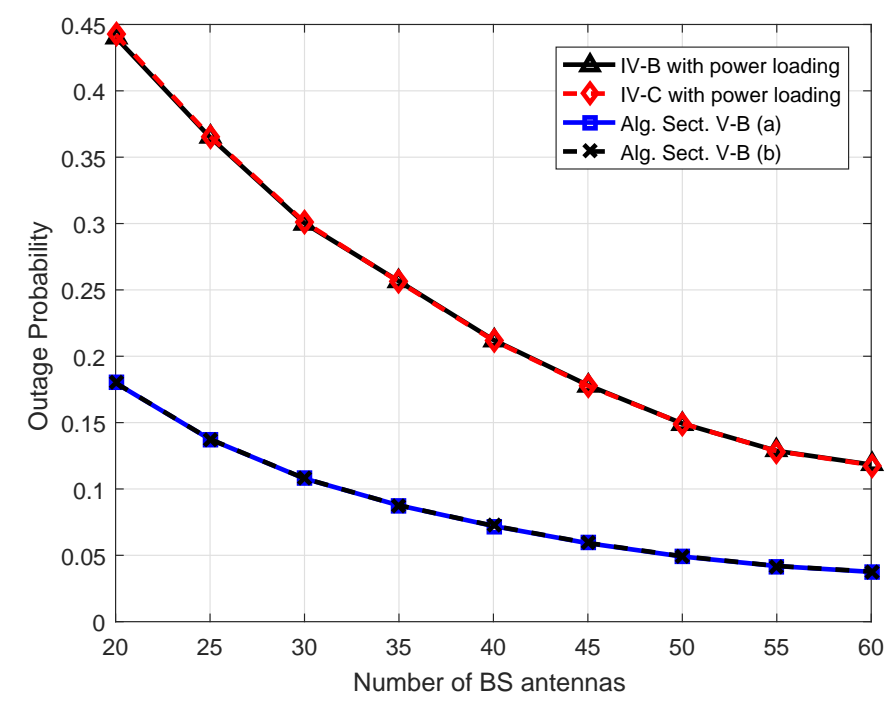

Figure 3.4: The outage probability versus the number of antennas for a system with 6 users, $\gamma=6 \mathrm{~dB}$, and $\sigma_{e_{k}}=0.1$.

We also observe that the power saving has almost no effect on the outage performance when we upper bound $r$ by 5 . However, significant power can be saved; the average actual transmitted powers used by the power saving algorithm (Alg. Sect. 3.5.2 (b))

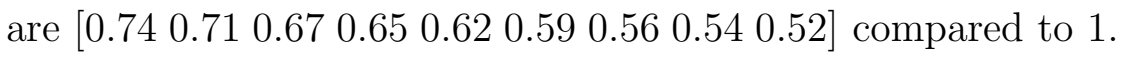

\subsection{Conclusion}

In this paper, a new offset-based approach is suggested for robust downlink beamforming. The approximation of the SINR outage constraint by an offset-based constraint enabled the formulation of the design problem as a convex problem. Further reductions can be obtained when the uncertainty size is small, allowing for an iterative closed-form solution. When the beamforming directions are defined in advance, the offset-based approach provide a power loading algorithm that provides significant 
performance and unique power control capabilities while using low number of computations. The shown performance gains, and the significant difference in computations suggest using the offset-based approach instead of the sphere bounding approach.

\section{A Choice of $r_{k}$}

From Cantelli's inequality, which is sometimes referred to as the one-sided Chebyshev inequality, we know that for any random variable $\mathbf{X}$ of a mean $\mu_{x}$ and variance $\sigma_{x}$,

$$
\operatorname{Prob}\left(\mathbf{X}-\mu_{x} \leq-r \sigma_{x}\right) \leq \frac{1}{1+r^{2}}
$$

Therefore, if we ensure that $\mu_{x} \geq r \sigma_{x}$ then $\operatorname{Prob}(\mathbf{X} \leq 0) \leq \frac{1}{1+r^{2}}$. Accordingly, if we design the beamformers such that $\mu_{f_{k}} \geq r_{k} \sigma_{f_{k}}$ then the outage probability is guaranteed to be less than $\frac{1}{1+r_{k}^{2}}$. However, for most practical applications this bound is quite loose, and such an approach for choosing $r_{k}$ can be quite conservative. Indeed, as we explained in Section 3.3, for small uncertainties the distribution of $f_{k}\left(\mathbf{e}_{k}\right)$ is close to being Gaussian. If it were in fact Gaussian, then if the beamformers are designed such that $\mu_{f_{k}} \geq r_{k} \sigma_{f_{k}}$ then the outage probability would be $Q\left(r_{k}\right)=\frac{1}{2} \operatorname{erfc}\left(\frac{r_{k}}{\sqrt{2}}\right)$, where $\operatorname{erfc}(\cdot)$ is the complementary error function.

\section{B Mean and variance derivations}

A Gaussian random variable $\mathbf{e}_{k} \sim \mathcal{C N}\left(\mathbf{m}_{k}, \mathbf{C}_{k}\right)$ can be represented as $\mathbf{e}_{k}=\mathbf{m}_{k}+$ $\mathbf{C}_{k}^{1 / 2} \hat{\mathbf{e}}_{k}$, where $\hat{\mathbf{e}}_{k} \backsim \mathcal{C N}(0, \mathbf{I})$. Using that representation we can write 


$$
\begin{aligned}
\mu_{f_{k}} & =\mathbb{E}\left\{f_{k}\left(\mathbf{e}_{k}\right)\right\} \\
& =\left(\mathbf{h}_{e_{k}}+\mathbf{m}_{k}\right)^{H} \mathbf{Q}_{k}\left(\mathbf{h}_{e_{k}}+\mathbf{m}_{k}\right)-\sigma_{k}^{2}+\mathbb{E}\left\{\hat{\mathbf{e}}_{k}^{H} \mathbf{C}_{k}^{1 / 2} \mathbf{Q}_{k} \mathbf{C}_{k}^{1 / 2} \hat{\mathbf{e}}_{k}\right\} \\
& =\left(\mathbf{h}_{e_{k}}+\mathbf{m}_{k}\right)^{H} \mathbf{Q}_{k}\left(\mathbf{h}_{e_{k}}+\mathbf{m}_{k}\right)-\sigma_{k}^{2}+\mathbf{w}_{k}^{H} \mathbf{C}_{k} \mathbf{w}_{k} / \gamma_{k}-\sum_{j \neq k} \mathbf{w}_{j}^{H} \mathbf{C}_{k} \mathbf{w}_{j}^{H} .
\end{aligned}
$$

The variance can be expressed as

$$
\begin{aligned}
\sigma_{f_{k}}^{2} & =\operatorname{var}\left\{f_{k}\left(\mathbf{e}_{k}\right)\right\} \\
& =\operatorname{var}\left\{2 \operatorname{Re}\left(\hat{\mathbf{e}}_{k}^{H} \mathbf{C}_{k}^{1 / 2} \mathbf{Q}_{k}\left(\mathbf{h}_{e_{k}}+\mathbf{m}_{k}\right)\right)+\hat{\mathbf{e}}_{k}^{H} \mathbf{C}_{k}^{1 / 2} \mathbf{Q}_{k} \mathbf{C}_{k}^{1 / 2} \hat{\mathbf{e}}_{k}\right\} \\
& =2\left(\mathbf{h}_{e_{k}}+\mathbf{m}_{k}\right)^{H} \mathbf{C}_{k}^{1 / 2} \mathbf{Q}_{k}^{2} \mathbf{C}_{k}^{1 / 2}\left(\mathbf{h}_{e_{k}}+\mathbf{m}_{k}\right)+\operatorname{var}\left\{\hat{\mathbf{e}}_{k}^{H} \mathbf{C}_{k}^{1 / 2} \mathbf{Q}_{k} \mathbf{C}_{k}^{1 / 2} \hat{\mathbf{e}}_{k}\right\}+0^{*} \\
& =2\left(\mathbf{h}_{e_{k}}+\mathbf{m}_{k}\right)^{H} \mathbf{C}_{k}^{1 / 2} \mathbf{Q}_{k}^{2} \mathbf{C}_{k}^{1 / 2}\left(\mathbf{h}_{e_{k}}+\mathbf{m}_{k}\right)+\operatorname{tr}\left(\mathbf{C}_{k}^{1 / 2} \mathbf{Q}_{k} \mathbf{C}_{k}^{1 / 2}\right)^{2}
\end{aligned}
$$

where $[\mathbf{A}]_{i j}$ denotes the $(i, j)$ th element of the matrix $\mathbf{A}$, and tr denotes the trace function. At the point marked with the asterisk we have used the fact that the expectation of the cross terms is equal to zero. This is true because $\mathbb{E}\left\{2 \operatorname{Re}\left(\mathbf{e}_{k}^{H} \mathbf{Q}_{k}\left(\mathbf{h}_{e_{k}}+\right.\right.\right.$ $\left.\left.\left.\mathbf{m}_{k}\right)\right)\left(\hat{\mathbf{e}}_{k}^{H} \mathbf{C}_{k}^{1 / 2} \mathbf{Q}_{k} \mathbf{C}_{k}^{1 / 2} \hat{\mathbf{e}}_{k}\right)\right\}$ consists of terms containing either similar or different components from the $\hat{\mathbf{e}}_{k}$ vector. Since $\hat{\mathbf{e}}_{k}$ has a zero mean, all terms with different indices will have a zero mean, while terms of similar indexes will take the form of a complex Gaussian raised to the power of three, which also have zero mean. 


\title{
Chapter 4
}

\section{Low-Complexity Robust MISO \\ Downlink Precoder Design With}

\section{Per-Antenna Power Constraints}

\begin{abstract}
This paper considers the design of the beamformers for a multiple-input singleoutput (MISO) downlink system that seeks to mitigate the impact of the imperfections in the channel state information (CSI) that is available at the base station (BS). The goal of the design is to minimize the outage probability of specified signal-tointerference-and-noise ratio (SINR) targets, while satisfying per-antenna power constraints (PAPCs), and to do so at a low computational cost. Based on insights from the offset maximization technique for robust beamforming, and observations regarding the structure of the optimality conditions, low-complexity iterative algorithms that involve the evaluation of closed-form expressions are developed. To further reduce the computational cost, algorithms are developed for per-antenna power-constrained
\end{abstract}


variants of the zero-forcing (ZF) and maximum ratio transmission (MRT) beamforming directions. In the MRT case, our low-complexity version for systems with a large number of antennas may be of independent interest. The proposed algorithms are extended to systems with both PAPCs and a total power constraint. Simulation results show that the proposed robust designs can provide substantial gains in the outage probability while satisfying the PAPCs.

\subsection{Introduction}

The spatial multiplexing capabilities of base stations (BSs) with multiple antennas offer the potential for substantial gains in the quality of service (QoS) that can be offered to users in a downlink system (e.g., Gesbert et al., 2007). In particular, linear beamforming schemes have been developed to simultaneously serve multiple users at their requested signal-to-interference-and-noise ratio (SINR) targets (Bengtsson and Ottersten, 2001; Rashid-Farrokhi et al., 1998a; Wiesel et al., 2006; Schubert and Boche, 2005; Bjornson et al., 2014). However, the performance of those beamforming schemes can be quite sensitive to the accuracy of the channel state information (CSI) that is available at the BS. Since that information is typically obtained through estimation on the uplink (in time division duplexing, TDD, systems) or through estimation on the downlink and quantized feedback (in frequency division duplexing, FDD, systems), the CSI at the BS is inherently uncertain. That observation has spawned the development of a variety of design strategies that incorporate different models for the uncertainty into the design. One strategy is to require the requested SINR to be met for all channels that are within a specified distance of the BS's model for the channel (Pascual-Iserte et al., 2006; Shenouda and Davidson, 2007; Zheng 
et al., 2008; Vucic and Boche, 2009a; Shenouda and Davidson, 2008b; Wang et al., 2014). However, in many scenarios that is a rather conservative approximation of the outage that occurs in practice. Furthermore, although this strategy, or a mild approximation thereof, often results in a convex optimization problem for finding the beamformers, the computational cost of solving those problems can be quite significant. Fortunately, different approaches to approximating the outage probability can yield alternative design strategies that provide excellent performance in practice, even when the uncertainties in the CSI are quite substantial, and do so in a computationally inexpensive way. One such strategy is the offset maximization algorithm (Medra et al., 2016), in which the beamformers are designed to maximize a carefully structured offset on the performance specification (see Section 4.2.2).

The above-mentioned design strategies seek to jointly design the beamforming directions and the power allocated to each direction. However, significant reductions in the computational cost can be obtained by computing the beamforming directions using a (computationally cheap) conventional technique and then developing a robust power loading algorithm. The beamforming directions in this approach are typically chosen to be either the maximum ratio transmission (MRT) (Lo, 1999) or zero-forcing (ZF) directions (Spencer et al., 2004). For the case of additive Gaussian uncertainties in the BS's CSI, single-integral expressions for the outage probability can be obtained (Al-Naffouri et al., 2016) and an effective algorithm for finding the power loading that minimizes the power required to meet the specified outage constraint has been developed (Sohrabi and Davidson, 2016). However, that algorithm is rather computationally expensive. (Medra and Davidson, 2015a) used insights from bounds 
on the cumulative distributive function to develop a new robust power loading technique that provides performance close to that of the optimal algorithm in (Sohrabi and Davidson, 2016), but has significantly lower computational cost.

The existing literature on robust downlink beamforming has tended to focus on designs that impose a constraint on the total power transmitted by the BS. In practice, each antenna will typically be driven by its own power amplifier, and hence the design ought to include constraints on the power transmitted from each antenna, as well as the total power. In the case of perfect CSI, a number of downlink beamforming algorithms that incorporate per-antenna power constraints (PAPCs) have been developed (Shen and Lok, 2014; Dartmann et al., 2013; Liu and Chen, 2009; Yazarel and Aktas, 2007; Yu and Lan, 2007). For robust beamforming designs that can be formulated as convex problems (e.g., Zheng et al., 2008; Shenouda and Davidson, 2007; Wang et al., 2014) and are solved using generic solvers, incorporating these additional constraints is quite straightforward. However, doing so increases the computational cost of what are, in comparison to the perfect CSI case, already quite expensive algorithms. The goal of this paper is to develop robust beamforming designs that incorporate PAPCs and have reasonable computational costs. Our technique is based on insights developed from the offset maximization approach to robust beamforming (Medra et al., 2016), a closely related power loading technique (Medra and Davidson, 2015a), and observations regarding the structure of the optimality conditions for the design problem. These observations enable us to develop a low-complexity dual update optimization startegy related to that by Yu and Lan (2007) that involves the evaluation of a sequence of closed-form expressions. After extending that algorithm 
to systems that have both PAPCs and a total power constraint, we make the observation that a large fraction of the computational cost arises from the design of the beamforming directions. To reduce that cost, we develop PAPCed variants of the $\mathrm{ZF}$ and MRT directions, and show how these can be incorporated into our design approach. Furthermore, we develop a low-complexity version of our PAPCed MRT beamforming algorithm for "massive MIMO" systems with a large number of antennas. As scaling techniques for large MRT beamformers have been recently proposed (Feng and Jing, 2016), that algorithm may be of independent interest.

\subsection{System model and design approach}

We consider a narrowband multiple-input single-output (MISO) downlink in which an $N_{t}$-antenna BS sends independent messages to $K$ single-antenna users. The transmitted signal at a given signalling instant is constructed using linear beamforming

as $\mathbf{x}=\sum_{k=1}^{K} \mathbf{w}_{k} s_{k}$, where $s_{k}$ is the power-normalized data symbol for user $k$, and $\mathbf{w}_{k}$ is the associated beamformer. In some settings we will refer to $\mathbf{u}_{k}=\mathbf{w}_{k} /\left\|\mathbf{w}_{k}\right\|$ as the direction of the beamformer, and $\beta_{k}=\mathbf{w}_{k}^{H} \mathbf{w}_{k}$ as the power allocated to that direction. That enables us to write

$$
\mathbf{w}_{k}=\sqrt{\beta_{k}} \mathbf{u}_{k} .
$$

The received signal at user $k$ can be written as

$$
y_{k}=\mathbf{h}_{k}^{H} \mathbf{w}_{k} s_{k}+\sum_{j \neq k} \mathbf{h}_{k}^{H} \mathbf{w}_{j} s_{j}+n_{k}
$$


where $\mathbf{h}_{k}^{H}$ denotes the channel between the BS and receiver $k$, and $n_{k}$ represents the additive zero-mean circular complex Gaussian noise at that user.

In the problems that we will consider, each user specifies the SINR that it will require in order to support the service that it desires. This constraint takes the form

$$
\operatorname{SINR}_{k}=\frac{\mathbf{h}_{k}^{H} \mathbf{w}_{k} \mathbf{w}_{k}^{H} \mathbf{h}_{k}}{\mathbf{h}_{k}^{H}\left(\sum_{j \neq k} \mathbf{w}_{j} \mathbf{w}_{j}^{H}\right) \mathbf{h}_{k}+\sigma_{k}^{2}} \geq \gamma_{k},
$$

where $\sigma_{k}^{2}$ is the noise variance at receiver $k$, and $\gamma_{k}$ is the required SINR. We will find it convenient to rewrite that constraint as

$$
\mathbf{h}_{k}^{H} \mathbf{Q}_{k} \mathbf{h}_{k}-\sigma_{k}^{2} \geq 0
$$

where

$$
\mathbf{Q}_{k}=\mathbf{w}_{k} \mathbf{w}_{k}^{H} / \gamma_{k}-\sum_{j \neq k} \mathbf{w}_{j} \mathbf{w}_{j}^{H} .
$$

If we denote the signal transmitted from antenna $i$ by $x_{i}$, then the power constraint on the BS as a whole can be written as $\sum_{i=1}^{N_{t}} E\left\{\left|x_{i}\right|^{2}\right\}=\sum_{k=1}^{K} \mathbf{w}_{k}^{H} \mathbf{w}_{k} \leq P_{t}$, where we have used the assumptions that the messages are independent and that the symbols $s_{k}$ are normalized. If we let $p_{i}$ denote the maximum power that can be transmitted from antenna $i$, the PAPC can be written as $E\left\{\left|x_{i}\right|^{2}\right\}=\left[\sum_{k=1}^{K} \mathbf{w}_{k} \mathbf{w}_{k}^{H}\right]_{i, i} \leq p_{i}$, where $[\cdot]_{i, i}$ denotes the $(i, i)$ th entry of the given matrix.

In order for a BS to be able to evaluate whether a candidate set of beamformers $\left\{\mathbf{w}_{k}\right\}_{k=1}^{K}$ satisfies the $K$ SINR constraints in (4.2), the BS must know each channel vector $\mathbf{h}_{k}$ (e.g., Bengtsson and Ottersten, 2001). However, typically the BS will only have an estimate of each channel, denoted $\mathbf{h}_{e_{k}}$. To incorporate the uncertainty in that channel estimate into the design, we will postulate a conditional distribution, 
$p\left(\mathbf{h}_{k} \mid \mathbf{h}_{e_{k}}\right)$, and convert the deterministic QoS constraint $\operatorname{SINR}_{k} \geq \gamma_{k}$ into the chance constraint $\operatorname{Prob}\left(\operatorname{SINR}_{k} \geq \gamma_{k}\right) \geq 1-\delta_{k}$, where $\delta_{k}$ is the required outage probability. In this paper, we will model the uncertainty additively; i.e.,

$$
\mathbf{h}_{k}=\mathbf{h}_{e_{k}}+\mathbf{e}_{k},
$$

with $\mathbf{e}_{k}$ having zero-mean and being independent of the channel and data. Our results will focus on the case where $\mathbf{e}_{k}$ is a zero-mean circular Gaussian random variable of covariance $\sigma_{e_{k}}^{2} \mathbf{I}$. Among a number of scenarios, that model is appropriate in certain TDD systems in which channels are estimated during the uplink training phase.

\subsubsection{Design approach}

With the uncertainty modeled as described above, one approach to the design of the downlink beamformers $\mathbf{w}_{k}$ is to seek to minimize the probability of outage of the SINR targets, subject to a total power constraint and PAPCs; i.e.,

$$
\begin{aligned}
\min _{\mathbf{w}_{k}, \delta_{k}} \max _{k} & \delta_{k} \\
\text { s.t. } & \sum_{k} \mathbf{w}_{k}^{H} \mathbf{w}_{k} \leq P_{t}, \\
& {\left[\sum_{k=1}^{K} \mathbf{w}_{k} \mathbf{w}_{k}^{H}\right]_{i, i} \leq p_{i}, \quad \forall i, } \\
& \operatorname{Prob}\left(\operatorname{SINR}_{k} \geq \gamma_{k}\right) \geq 1-\delta_{k}, \quad \forall k .
\end{aligned}
$$

This problem is hard to solve even without the PAPCs. However, in the case that the PAPCs are omitted, the offset maximization algorithm (Medra et al., 2016) is a low-complexity algorithm that has been shown to provide good performance. The 
goal of this paper is to use insights from the development of the offset maximization approach to develop an effective low-complexity algorithm for the PAPCed case. One observation that we will use is that the performance of the offset maximization approach can be improved by applying the robust power loading algorithm in (Medra and Davidson, 2015a) to the beamforming directions generated by the offset maximization. Doing so reveals that robust beamformers can be obtained with a computational cost that is similar to that of beamformer design in the perfect CSI case. (Many existing approaches to robust beamforming are much more expensive than the perfect CSI case (e.g., Shenouda and Davidson, 2008b; Wang et al., 2014).) However, like the perfect CSI case, it is the computation of the directions that dominate the computational cost. Therefore, we also propose to apply the principles that underlie the power loading by Medra and Davidson (2015a) to beamforming directions that can be computed more efficiently, such as PAPCed variants, derived herein, of the classical ZF and MRT directions; see Sections 4.4, and 4.5. In the latter case, a further approximation that is suitable for scenarios with a large number of antennas at the BS substantially reduces the computational cost, and has almost the same outage performance.

To lay the groundwork for the development of the proposed beamforming schemes, in the following subsections we briefly review the offset maximization approach to beamformer design under a total power constraint (Medra et al., 2016), and the lowcomplexity robust power loading technique for systems with a total power constraint that was developed by Medra and Davidson (2015a). 


\subsubsection{Offset maximization beamforming directions}

The offset maximization beamformers (Medra et al., 2016) can be found by solving the following problem:

$$
\begin{array}{rl}
r_{t}^{\star}=\max _{\mathbf{w}_{k}, r} & r \\
\text { s.t. } & \sum_{k=1}^{K} \mathbf{w}_{k}^{H} \mathbf{w}_{k} \leq P_{t}, \\
& \mathbf{h}_{e_{k}}^{H} \mathbf{Q}_{k} \mathbf{h}_{e_{k}}-\sigma_{k}^{2}-r \geq 0, \quad \forall k .
\end{array}
$$

It is implicit in (4.6c) that this algorithm tries to find the largest noise-plus-interference power each user can endure, under the total power constraint. Medra et al. (2016) developed an efficient method to solve (4.6) by considering the following problem, in which, for now, it is assumed that the optimal value for (4.6), $r_{t}^{\star}$, is known:

$$
\begin{aligned}
P^{\star}=\min _{\mathbf{w}_{k}} & \sum_{k} \mathbf{w}_{k}^{H} \mathbf{w}_{k} \\
\text { s.t. } & \mathbf{h}_{e_{k}}^{H} \mathbf{Q}_{k} \mathbf{h}_{e_{k}}-\sigma_{k}^{2}-r_{t}^{\star} \geq 0, \quad \forall k .
\end{aligned}
$$

It can be shown (Medra et al., 2016) that the optimal value of the problem in (4.7) is $P_{t}$, and that any set of beamformers that optimize (4.7) are also optimal for (4.6). Also, at optimality, all the constraints are satisfied with equality.

The advantage of the connection between problems (4.6) and (4.7) is that a highly efficient algorithm for the problem in (4.7) with $r=0$ (i.e., the perfect CSI case) was developed by Rashid-Farrokhi et al. (1998a); (see also Bjornson et al., 2014). That algorithm can be extended to jointly find the optimal beamformers and the optimal offset, $r_{t}^{\star}$, for the problem in (4.6). In particular, if we let $\nu_{k}$ denote the Lagrange 
multiplier for the SINR constraint in (4.7b), then from the KKT conditions of (4.7) we can find the offset maximization directions by solving the eigen problem

$$
\mathbf{u}_{k}=\left(\frac{\nu_{k}}{\gamma_{k}} \mathbf{h}_{e_{k}} \mathbf{h}_{e_{k}}^{H}-\sum_{j \neq k} \nu_{j} \mathbf{h}_{e_{j}} \mathbf{h}_{e_{j}}^{H}\right) \mathbf{u}_{k}
$$

where the Lagrange multipliers must satisfy the fixed-point relation

$$
\nu_{k}^{-1}=\mathbf{h}_{e_{k}}^{H}\left(\mathbf{I}_{N_{t}}+\sum_{j} \nu_{j} \mathbf{h}_{e_{j}} \mathbf{h}_{e_{j}}^{H}\right)^{-1} \mathbf{h}_{e_{k}}\left(1+\frac{1}{\gamma_{k}}\right) .
$$

Since (4.8) can be solved using a power method, the complexity of finding the directions is dominated by the matrix inversion in (4.9), which requires $\mathcal{O}\left(N_{t}^{3}\right)$ operations. Having found those directions, the offset maximization power loading and the optimal offset can be found by solving the $K+1$ linear equations that arise when the constraints in (4.6b) and (4.6c) hold with equality.

\subsubsection{Robust power loading}

The offset maximization algorithm described above uses the same offset $r$ to increase the robustness of each user to channel uncertainty. The goal of robust power loading approach by Medra and Davidson (2015a) is to provide a computationally-efficient way to adapt the offset to the characteristics of each user's channel. For an arbitrary set of beamforming directions $\left\{\mathbf{u}_{k}\right\}$, the generic power loading problem can be stated 
as

$$
\begin{array}{ll}
\min _{\beta_{k}, \delta_{k}} & \max _{k} \quad \delta_{k} \\
\text { s.t. } & \sum_{k=1}^{K} \beta_{k} \leq P_{t}, \\
& \operatorname{Prob}\left(\operatorname{SINR}_{k} \geq \gamma_{k}\right) \geq 1-\delta_{k}, \quad \forall k .
\end{array}
$$

The derivation of the algorithm developed by Medra and Davidson (2015a) for producing good solutions to (4.10) begins by observing the under the additive uncertainty model in (4.4), the probability that $\mathrm{SINR}_{k} \geq \gamma_{k}$ is equal to the probability that

$$
f_{k}\left(\mathbf{e}_{k}\right)=\mathbf{h}_{e_{k}}^{H} \mathbf{Q}_{k} \mathbf{h}_{e_{k}}+2 \operatorname{Re}\left(\mathbf{e}_{k}^{H} \mathbf{Q}_{k} \mathbf{h}_{e_{k}}\right)+\mathbf{e}_{k}^{H} \mathbf{Q}_{k} \mathbf{e}_{k}-\sigma_{k}^{2} \geq 0 .
$$

If we assume that the norms of the errors $\mathbf{e}_{k}$ are small, as they will need to be for reliable operation (Jindal, 2006), then we can approximate the quadratic term $\mathbf{e}_{k}^{H} \mathbf{Q}_{k} \mathbf{e}_{k}$ by a Gaussian random variable of the same mean and variance. In that case, the distribution of $f_{k}\left(\mathbf{e}_{k}\right)$ becomes Gaussian. (Recall that we are focusing on the case where $\mathbf{e}_{k}$ is Gaussian, with zero mean and of covariance $\sigma_{e_{k}}^{2} \mathbf{I}$; cf. (4.4).) Under that approximation, if we design the power loading so that the mean, $\mu_{f_{k}}$, of $f_{k}\left(\mathbf{e}_{k}\right)$ is a significant multiple of its standard deviation, $\sigma_{f_{k}}$, then that user will achieve a low outage probability. Indeed, we can choose a value for that multiple so that the target outage probability is guaranteed to be satisfied (e.g., Pascual-Iserte et al., 2006). We also note that the optimal solution of (4.10) has equal values for $\delta_{k}$. If that were not the case, the user(s) with higher outage probability could be allocated more power and the other user(s) less, which would reduce the objective value, and 
thus contradict the assumed optimality. Therefore, it is natural to choose the same multiple, $r$, for each user in the approximation of the outage constraint in (4.10c). The resulting approximation of the problem in (4.10) can be written as (Medra and Davidson, 2015a)

$$
\begin{array}{ll}
\max _{\beta_{k}, r} & r \\
\text { s.t. } & \sum_{k=1}^{K} \beta_{k} \leq P_{t}, \\
& \mu_{f_{k}} \geq r \sigma_{f_{k}}, \quad \forall k .
\end{array}
$$

From the definition of $f_{k}\left(\mathbf{e}_{k}\right)$ in (4.11) and the channel uncertainty model in (4.4), it can be shown that

$$
\mu_{f_{k}}=\mathbf{h}_{e_{k}}^{H} \mathbf{Q}_{k} \mathbf{h}_{e_{k}}-\sigma_{k}^{2}+\sigma_{e}^{2} \beta_{k}\left(1 / \gamma_{k}+1\right)-\sigma_{e}^{2} P_{t},
$$

which is linear in the design variables $\left\{\beta_{k}\right\}_{k=1}^{K}$. (Recall from (4.3) that $\mathbf{Q}_{k}=\beta_{k} \mathbf{u}_{k} \mathbf{u}_{k}^{H} / \gamma_{k}-$ $\sum_{j \neq k} \beta_{j} \mathbf{u}_{j} \mathbf{u}_{j}^{H}$.) Similarly, we have that

$$
\sigma_{f_{k}}^{2}=\operatorname{var}\left\{f_{k}\left(\mathbf{e}_{k}\right)\right\}=2 \sigma_{e}^{2} \mathbf{h}_{e_{k}}^{H} \mathbf{Q}_{k}^{2} \mathbf{h}_{e_{k}}+\sigma_{e}^{4} \operatorname{tr}\left(\mathbf{Q}_{k}^{2}\right) .
$$

The structure of the problem in (4.12) is such that the constraints hold with equality at optimality (Medra and Davidson, 2015a). Since $\sigma_{f_{k}}$ is not a linear function in $\boldsymbol{\beta}$, that results in a set of non-linear equations for the power loading. The following iterative linearization technique has been shown by Medra and Davidson (2015a) to be an effective way to obtain good solutions to (4.12): 
1. Initialize each $\sigma_{f_{k}}=1$.

2. Find $\left\{\beta_{k}\right\}$ and $r$ by solving the set of linear equations that arise from equality in (4.12b) and (4.12c) for the current values of $\sigma_{f_{k}}$, where $\mu_{f_{k}}$ is defined in (5.9).

3. Update each $\sigma_{f_{k}}$ using (4.14).

4. Return to (2) until a convergence criterion is satisfied.

We note that the matrix that relates $\left\{\beta_{k}\right\}$ to $\sigma_{f_{k}}$ and $r$ in step 2 is constant, and, accordingly, we need only invert this matrix once (Medra and Davidson, 2015a). In practice, this algorithm converges quickly with a high probability (Medra and Davidson, 2015a). Medra and Davidson (2016) showed that this algorithm provides very similar performance to the optimal power loading by Sohrabi and Davidson (2016), and at a cost that is dominated by the $\mathcal{O}\left(K^{3}\right)$ operations that result from the initial matrix inversion.

\subsection{Offset maximization designs with PAPCs}

To simplify the development of the proposed robust beamforming technique, we will first consider the addition of PAPCs to the offset maximization problem in (4.6). We will then modify the resulting algorithm using insights from the above robust power loading algorithm.

When we add the PAPCs to the offset maximization problem in (4.6), the design 
problem becomes

$$
\begin{array}{rl}
r_{t p a}^{\star}=\max _{\mathbf{w}_{k}, r} & r \\
\text { s.t. } \quad & \sum_{k=1}^{K} \mathbf{w}_{k}^{H} \mathbf{w}_{k} \leq P_{t}, \\
& {\left[\sum_{k=1}^{K} \mathbf{w}_{k} \mathbf{w}_{k}^{H}\right]_{i, i} \leq p_{i}, \quad \forall i,} \\
& \mathbf{h}_{e_{k}}^{H} \mathbf{Q}_{k} \mathbf{h}_{e_{k}}-\sigma_{k}^{2}-r \geq 0, \quad \forall k .
\end{array}
$$

Although the formulation in (4.15) is not convex, it can be transformed in a straightforward way into a second order cone program, using the technique that was used for the case of perfect CSI (cf., Wiesel et al., 2006; Yu and Lan, 2007). While that formulation can be solved using a generic interior point method (e.g., Boyd and Vandenberghe, 2004), such generic methods do not exploit the structure of the problem, and the development of tailored algorithms that do exploit the structure offers the potential for improved computational efficiency.

In the following subsections, we will first develop a low-complexity algorithm for the case where we have PAPCs only, with no total power constraint. Then we will tackle the general problem with both types of power constraints. The development will use insights from algorithms developed for the perfect CSI case (Yu and Lan, 2007) and insights from the robust power loading algorithm described in Section 4.2.3. 


\subsubsection{Dominant PAPCs}

If $P_{t}>\sum_{i=1}^{N_{t}} p_{i}$, the total power constraint can never be active and the problem in (4.15) can be rewritten as

$$
\begin{array}{rl}
r_{p a}^{\star}=\max _{\mathbf{w}_{k}, r} & r \\
\text { s.t. } & {\left[\sum_{k=1}^{K} \mathbf{w}_{k} \mathbf{w}_{k}^{H}\right]_{i, i} \leq p_{i}, \quad \forall i} \\
& \mathbf{h}_{e_{k}}^{H} \mathbf{Q}_{k} \mathbf{h}_{e_{k}}-\sigma_{k}^{2}-r \geq 0, \quad \forall k .
\end{array}
$$

Motivated by the way that a customized algorithm for (4.7) was adapted (Medra et al., 2016) to solve the problem in (4.6), we consider the following problem in which, for now, $r_{p a}^{\star}$ is presumed to be known,

$$
\begin{array}{ll}
\min _{\mathbf{w}_{k}, \alpha} & \alpha \sum_{i=1}^{N_{t}} p_{i} \\
\text { s.t. } & {\left[\sum_{k=1}^{K} \mathbf{w}_{k} \mathbf{w}_{k}^{H}\right]_{i, i} \leq \alpha p_{i}, \quad \forall i,} \\
& \mathbf{h}_{e_{k}}^{H} \mathbf{Q}_{k} \mathbf{h}_{e_{k}}-\sigma_{k}^{2}-r_{p a}^{\star} \geq 0, \quad \forall k .
\end{array}
$$

In the context of (4.17), the constant term $\sum_{i=1}^{N_{t}} p_{i}$ in the objective is superfluous, but it will simplify the interpretation of the Lagrangian. Using arguments analogous to those by Medra et al. (2016); Yu and Lan (2007), it can be shown that any set of beamformers that is optimal for (4.17) is also optimal for (4.16), and the optimal value of $\alpha$ in (4.17) is one.

Now, let $q_{i}$ denote the dual variable of the $i$ th condition in (4.17b) and $\nu_{k}$ denote the dual variable of the $k$ th condition in (4.17c). Let us also define the diagonal matrix $\hat{\mathbf{Q}}$, such that $[\hat{\mathbf{Q}}]_{i, i}=q_{i}$. These definitions enable us to write the Lagrangian 
of the problem in (4.17) as

$$
\begin{aligned}
\mathcal{L}\left(\mathbf{w}_{k}, \alpha, \nu_{k}, q_{i}\right)=\sum_{k=1}^{K} \nu_{k}\left(\sigma_{k}^{2}\right. & \left.+r_{p a}^{\star}\right)+\alpha\left(\sum_{i=1}^{N_{t}} p_{i}-\sum_{i=1}^{N_{t}} q_{i} p_{i}\right) \\
& +\sum_{k=1}^{K} \mathbf{w}_{k}^{H}\left(\hat{\mathbf{Q}}+\sum_{j \neq k} \nu_{j} \mathbf{h}_{e_{j}} \mathbf{h}_{e_{j}}^{H}-\nu_{k} / \gamma_{k} \mathbf{h}_{e_{k}} \mathbf{h}_{e_{k}}^{H}\right) \mathbf{w}_{k} .
\end{aligned}
$$

Using the notion of complementary slackness, since the optimal value of $\alpha$ is one, at optimality we have that $\sum_{i=1}^{N_{t}} p_{i}-\sum_{i=1}^{N_{t}} q_{i} p_{i}=0$. Also, at optimality we have $\hat{\mathbf{Q}}+\sum_{j \neq k} \nu_{j} \mathbf{h}_{e_{j}} \mathbf{h}_{e_{j}}^{H}-\nu_{k} / \gamma_{k} \mathbf{h}_{e_{k}} \mathbf{h}_{e_{k}}^{H} \succeq \mathbf{0}$, with $\mathbf{w}_{k}$ lying in the null space of this matrix. This can be simplified to show that $\mathbf{w}_{k}$ and

$$
\hat{\mathbf{w}}_{k}=\left(\hat{\mathbf{Q}}+\sum_{k} \nu_{k} \mathbf{h}_{e_{k}} \mathbf{h}_{e_{k}}^{H}\right)^{\dagger} \mathbf{h}_{e_{k}}
$$

where $(\cdot)^{\dagger}$ denotes the Moore-Penrose pseudo-inverse, should be in the same direction. Further simplifications show that the dual variable $\nu_{k}$ in (4.19) should satisfy the fixed point equation

$$
\nu_{k}^{-1}=\mathbf{h}_{e_{k}}^{H}\left(\hat{\mathbf{Q}}+\sum_{j} \nu_{j} \mathbf{h}_{e_{j}} \mathbf{h}_{e_{j}}^{H}\right)^{\dagger} \mathbf{h}_{e_{k}}\left(1+\frac{1}{\gamma_{k}}\right)
$$

From (4.20) we observe that if we were given the optimal $\hat{\mathbf{Q}}$, we could find the optimal values for $\left\{\nu_{k}\right\}$ using (4.20) and then the optimal directions $\left\{\mathbf{u}_{k}\right\}$ by normalizing the $\left\{\hat{\mathbf{w}}_{k}\right\}$ obtained using (4.19). After doing so, we could complete the solution of (4.17) by finding the optimal values for $\beta_{k}=\left\|\mathbf{w}_{k}\right\|^{2}$. That can be done by solving the set of $K$ linear equations that arise from the fact that at optimality (4.17c) holds with equality. (If this were not the case for condition $k$ in (4.17c), then the amplitude of $\mathbf{w}_{k}$ could be decreased which would allow a smaller value of $\alpha$ while satisfy all the other constraints.) To adapt that approach to solve (4.16), in the final step we must 
simultaneously solve for $\left\{\beta_{k}\right\}$ and $r_{p a}^{\star}$. To do so we observe that $r_{p a}^{\star}$ enters linearly into $(4.17 \mathrm{c})$, and hence all we need is one more linearly independent equation. To obtain that equation we observe that if $q_{i}>0$, then the $i$ th component of (4.17b) holds with equality. By summing over all the active constraints in (4.17b) we obtain the following equation

$$
\sum_{i, \forall q_{i} \neq 0}\left[\sum_{k=1}^{K} \beta_{k} \mathbf{u}_{k} \mathbf{u}_{k}^{H}\right]_{i, i}=\sum_{i, \forall q_{i} \neq 0} p_{i}
$$

In the case that all the $q_{i}$ are positive - a case that happens quite often - the equation in (4.21) simplifies to $\sum_{k=1}^{K} \beta_{k}=\sum_{i=1}^{N_{t}} p_{i}$.

To complete the algorithm, we need to develop a technique to determine the optimal Q. One strategy for doing so is to apply the projected subgradient technique developed by Yu and Lan (2007). That involves applying the update equation $\hat{\mathbf{Q}}^{n+1}=$ $\operatorname{proj}\left(\hat{\mathbf{Q}}^{n}+t_{n} \operatorname{diag}\left(\operatorname{diag}\left(\sum_{i} \mathbf{w}_{i} \mathbf{w}_{i}^{H}\right)\right)\right)$, where $\operatorname{proj}(\cdot)$ denotes the projection of a matrix on the space of diagonal positive semidefinite matrices that satisfy $\sum_{i=1}^{N_{t}} q_{i} p_{i}=\sum_{i=1}^{N_{t}} p_{i}$ and, consistent with the syntax used in MATLAB, when $\operatorname{diag}(\cdot)$ operates on a matrix it produces a vector containing the diagonal elements and when it operates on a vector it produces a diagonal matrix with the elements of the vector on the diagonal. The initialization parameters used by Yu and Lan (2007) were chosen to be $\hat{\mathbf{Q}}^{0}=\mathbf{I}$ and the step size chosen to be $t_{n}=1 / n$. Although this strategy converges, it can be quite slow (Yu and Lan, 2007). In this paper, we will refine the approach in two ways. First, in Appendix 4.A we develop a computationally cheap quasi-closed-form expression for the projection of $\hat{\mathbf{Q}}^{n+1}$ in a 2-norm sense. Second, based on insights from Scutari et al. (2014) we will choose a step size of the form $t_{n}=t_{n-1}-t_{n-1}^{2} / a$, for some positive scalar $a$. In addition, in Section 4.3 .3 we will identify a prediction 
step that can be used in the first iteration to accelerate the algorithm. One simple termination strategy is to stop the algorithm when $\left[\sum_{k} \mathbf{w}_{k} \mathbf{w}_{k}^{H}\right]_{i, i}-p_{i}<\epsilon_{i}, \forall i$, where $\epsilon_{i}$ is the maximum allowable violation of the power constraint for the $i$ th antenna. Following the above development, the algorithm can be summarized as shown in Algorithm 1.

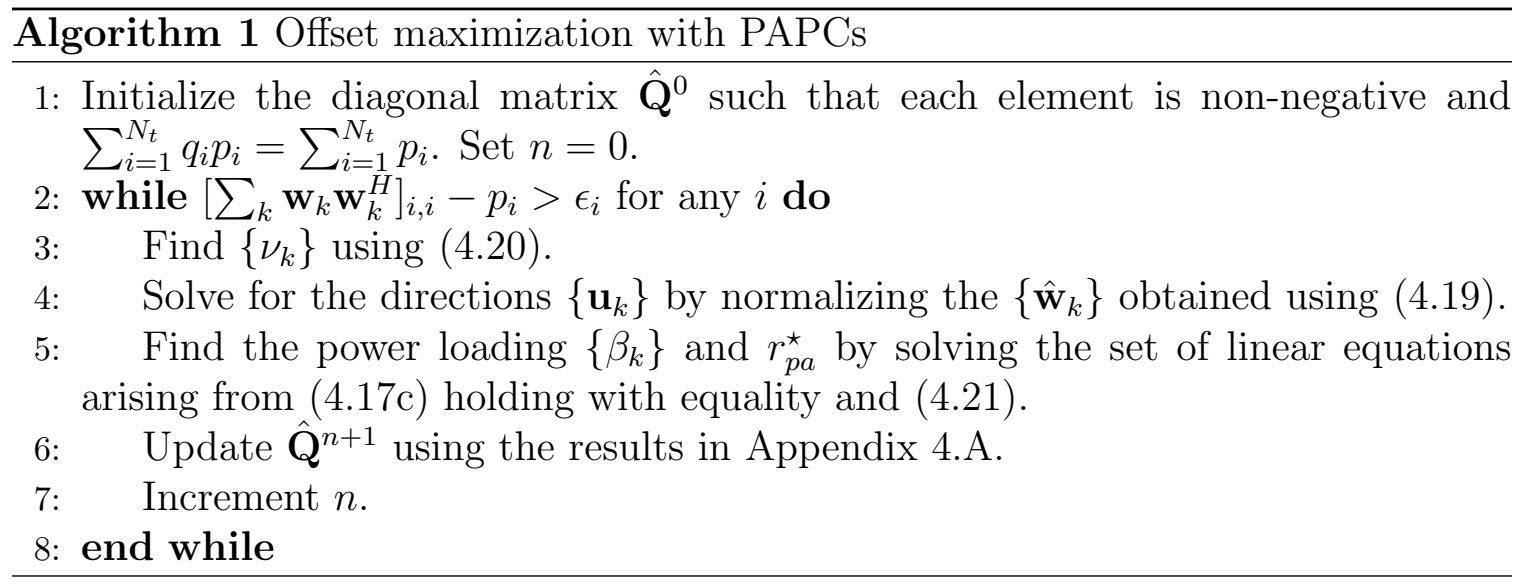

Having developed an efficient algorithm for the offset maximization problem with PAPCs, we now seek to incorporate the principles of the robust power loading discussed in Section 4.2.3. To do so, we note that in the offset maximization design, the directions are independent of the offset term $r$ in (4.16c); cf. (4.19) and (4.20). That suggests that we could simply modify the power loading step. Indeed, once the directions have been obtained in step 4 of Algorithm 1, we can replace the power loading in step 5 by the $\left\{\beta_{k}\right\}$ and $r^{\star}$ that solve (4.12). Those values can be found using the algorithm in Section 4.2.3 (see Medra and Davidson, 2015a). Incorporating that robust power loading algorithm into the framework of Algorithm 1 results in Algorithm 2. 


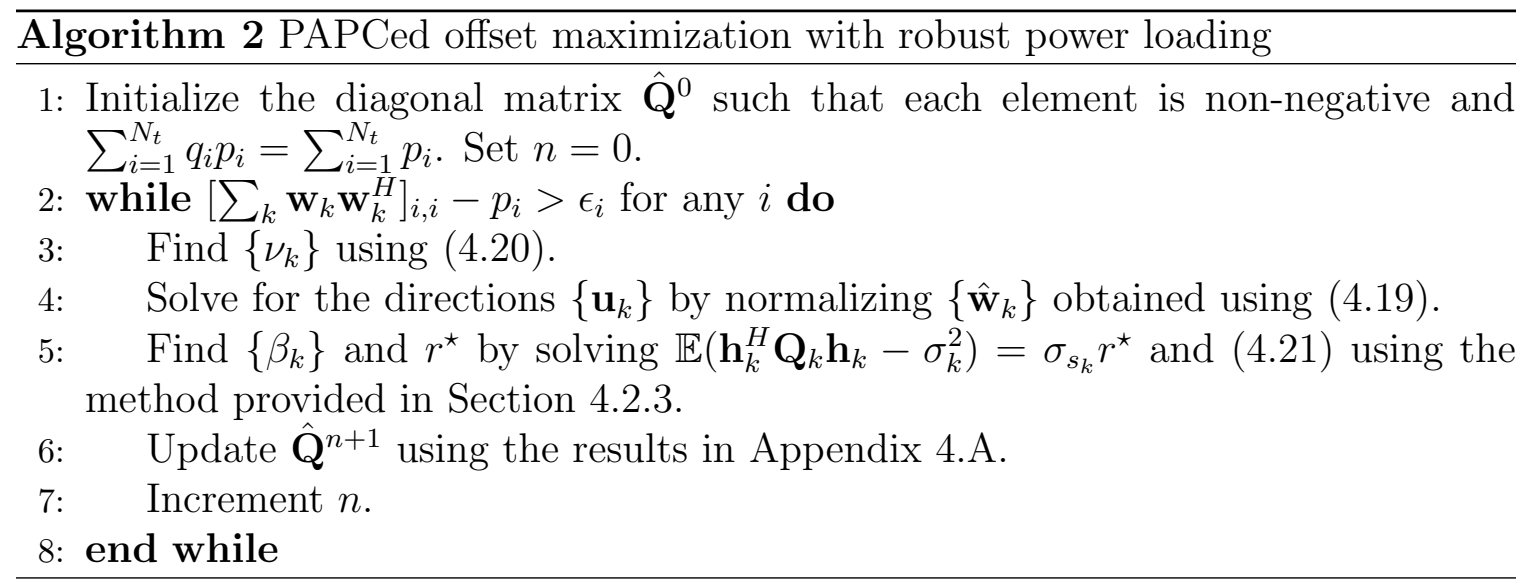

\subsubsection{Total and PAPCed algorithm}

Using the principles outlined in Section 4.2.2 and the previous section, we can develop an algorithm for solving the general problem in (4.15), which has PAPCs and a total power constraint. In this section, we will focus on the case when $P_{t}$ is sufficiently smaller than $\sum_{i} p_{i}$ to ensure that the total power constraint is active. (Otherwise, the problem can be solved by the techniques in the previous section.) Similar to the previous section, we will obtain the beamforming directions by normalizing the beamformers resulting from the following problem

$$
\begin{array}{ll}
\min _{\mathbf{w}_{k}} & \sum_{k=1}^{K} \mathbf{w}_{k}^{H} \mathbf{w}_{k} \\
\text { s.t. } & {\left[\sum_{j=1}^{K} \mathbf{w}_{j} \mathbf{w}_{j}^{H}\right]_{i, i} \leq p_{i}, \quad \forall i} \\
& \mathbf{h}_{e_{k}}^{H} \mathbf{Q}_{k} \mathbf{h}_{e_{k}}-\sigma_{k}^{2}-r_{t p a}^{\star} \geq 0, \quad \forall k,
\end{array}
$$

and then we will refine the power loading using the method described in Section 4.2.3. As in the previous development, the Lagrangian of (4.22) plays a key role. It can be 
written as

$$
\begin{aligned}
\mathcal{L}\left(\mathbf{w}_{k}, \nu_{k}, q_{i}\right)=\sum_{k=1}^{K} \nu_{k}\left(\sigma_{k}^{2}+r_{t p a}^{\star}\right)-\sum_{i=1}^{N_{t}} q_{i} p_{i}+ \\
\sum_{k=1}^{K} \mathbf{w}_{k}^{H}\left(\mathbf{I}+\hat{\mathbf{Q}}+\sum_{j \neq k} \nu_{j} \mathbf{h}_{e_{j}} \mathbf{h}_{e_{j}}^{H}-\nu_{k} / \gamma_{k} \mathbf{h}_{e_{k}} \mathbf{h}_{e_{k}}^{H}\right) \mathbf{w}_{k} .
\end{aligned}
$$

Using the KKT conditions, for a given value for $\hat{\mathbf{Q}}$ we can compute the corresponding directions and then the robust power loading in Section 4.2.3. Furthermore, the subgradient used in the previous section remains a subgradient in this case. However, the structure of the KKT conditions is simpler in this case, which results in a more straightforward projection for the $\hat{\mathbf{Q}}$ matrix. Indeed, since the only constraint on $q_{i}$ in this case is that it is non-negative, the update equation for $\hat{\mathbf{Q}}$ can be written as

$$
\hat{\mathbf{Q}}^{n+1}=\max \left(\hat{\mathbf{Q}}^{n}+t_{n} \operatorname{diag}\left(\operatorname{diag}\left(\sum_{k} \mathbf{w}_{k} \mathbf{w}_{k}^{H}\right)-\mathbf{p}\right), \mathbf{0}\right),
$$

where the maximum operator is defined element-wise, and $\mathbf{p}$ is the vector whose $i$ th element is $p_{i}$. Therefore, we can construct an algorithm that has a similar structure to that in Algorithm 2. Having said that, in the case of PAPCs only there is a strong likelehood that the PAPCs will be active at optimality, and hence it makes sense to initialize the algorithm with a positive definite matrix $\hat{\mathbf{Q}}^{0}$. In the general case, the PAPCs are less likely to be active at optimality, and hence we will initialize the algorithm with $\hat{\mathbf{Q}}^{0}=\mathbf{0}$. The resulting algorithm is provided in Algorithm 3. 


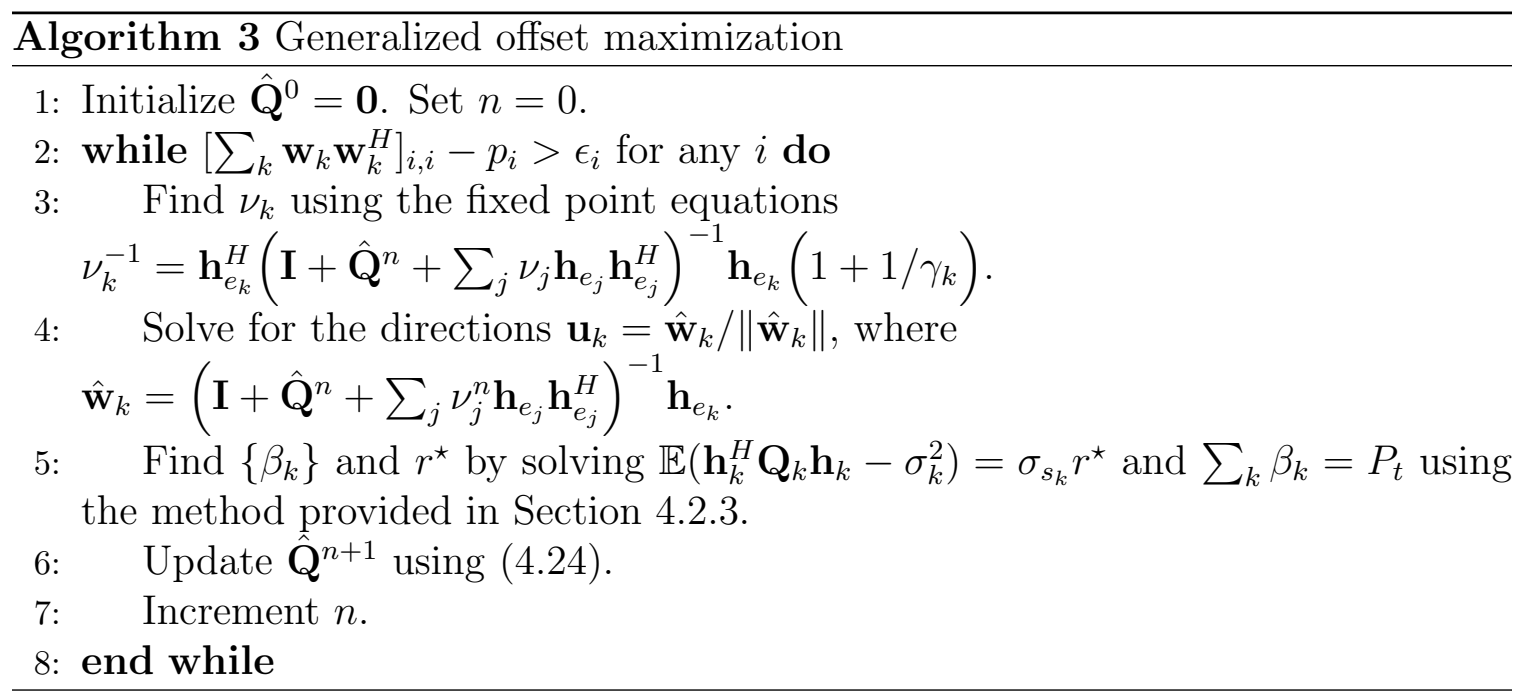

\subsubsection{Algorithm acceleration}

As will be apparent in the simulations in Section 4.6, the modified update in Appendix 4.A and the improved step size selection result in a substantial reduction of the number of iterations required over the number required using the choices made by Yu and Lan (2007). Furthermore, we have observed that $\hat{\mathbf{Q}}^{1}$ and the corresponding matrix $\hat{\mathbf{Q}}^{n}$ at the termination of the algorithm are typically closely related. If that relationship can be determined with reasonable accuracy, this observation suggests that a predictive step could be used to further reduce the number of iterations. As an example of what can be done, in Section 4.6 we illustrate how replacing $\hat{\mathbf{Q}}^{1}$ with a simple affine prediction, $\hat{\mathbf{Q}}_{p}^{1}$, of the terminating matrix $\hat{\mathbf{Q}}^{n}$ results in substantial reduction in the number of iterations. 


\subsection{Conventional ZF beamforming with per-antenna power constraints}

Even though the computational cost of each iteration of the PAPCed offset maximization beamforming algorithms in the previous section is dominated by terms that are only $\mathcal{O}\left(N_{t}^{3}\right)$, when the BS has a large number of antennas the resulting computational load can still be substantial. The dominating components arise from determining the beamforming directions, and the fact that these directions are updated at each iteration. That suggests that we may be able to develop lower cost algorithms for systems with a large number of antennas if we could find a way to simplify the computation of the beamforming directions. In this section we will do that by developing variants of the nominal ZF directions, and we will integrate them with the robust power loading technique while ensuring that the required PAPCs are satisfied. In the following section we will develop analogous techniques based on variants of the MRT directions. For the ZF case, the beamforming directions are obtained using techniques developed by Wiesel et al. (2008), but in the MRT case, the design of the beamforming directions appears to be new.

To develop PAPCed variants of the conventional ZF and MRT beamformers, we observe that in contrast to QoS-based designs, in which the SINR is controlled directly (e.g., (4.15d)), the conventional ZF and MRT designs focus on the desired signal power and interference components of the SINR separately. In particular,

given that the SINR for user $k$ is $\operatorname{SINR}_{k}=\frac{\mathbf{h}_{k}^{H} \mathbf{w}_{k} \mathbf{w}_{k}^{H} \mathbf{h}_{k}}{\mathbf{h}_{k}^{H}\left(\sum_{j \neq k} \mathbf{w}_{j} \mathbf{w}_{j}^{H}\right) \mathbf{h}_{k}+\sigma_{k}^{2}}$, if we were to maximize the minimum nominal received signal power subject to a total power constraint 
(i.e., $\max _{\left\{\mathbf{w}_{k}\right\}} \min _{k} \mathbf{h}_{e_{k}}^{H} \mathbf{w}_{k} \mathbf{w}_{k}^{H} \mathbf{h}_{e_{k}}$ subject to $\sum_{k} \mathbf{w}_{k}^{H} \mathbf{w}_{k} \leq P_{t}$ ) we would obtain beamformers that are a particular power loading of the nominal MRT directions. If we were to add the nominal ZF constraints on the interference into that problem (i.e., $\mathbf{h}_{e_{j}}^{H} \mathbf{w}_{k} \mathbf{w}_{k}^{H} \mathbf{h}_{e_{j}}=0, \forall k \neq j$ ), then we would obtain beamformers that are a particular power loading of the ZF directions (Wiesel et al., 2008). Due to the structure of the total power constraint, in many simple beamforming problems the optimization of the beamforming directions decouples from the power loading. That is indeed the case for our formulation for MRT and ZF beamforming directions. As an example, if we were to maximize the minimum value of $\mathbf{h}_{e_{k}}^{H} \mathbf{w}_{k} \mathbf{w}_{k}^{H} \mathbf{h}_{e_{k}} /\left\|\mathbf{h}_{e_{k}}\right\|^{2}$, which is the power of the signal transmitted in the direction of user $k$, rather than the power received by that user, we would obtain a set of beamformers in the MRT or ZF directions, but with a different power loading.

When the total power constraint is replaced by PAPCs, the optimization of the beamforming directions becomes coupled with the power loading and hence the choice of the metric to optimize changes both the power loading and the directions. While our approach will work for either metric, and indeed for several others, we will focus on the second metric $\mathbf{h}_{e_{k}}^{H} \mathbf{w}_{k} \mathbf{w}_{k}^{H} \mathbf{h}_{e_{k}} /\left\|\mathbf{h}_{e_{k}}\right\|^{2}$. The rationale for this choice is that while the received signal power is suitable for the ZF problem in the perfect CSI case, where the ZF constraints will eliminate the interference (Wiesel et al., 2008), it can be quite sensitive to the interference incurred due to channel estimation errors. (This is illustrated in our simulation results in Section 4.6.) Accordingly, we define the normalized channel directions $\mathbf{h}_{n_{k}}=\mathbf{h}_{e_{k}} /\left\|\mathbf{h}_{e_{k}}\right\|$ and we formulate the following generic problem to obtain PAPCed versions of the conventional beamformers 


$$
\begin{array}{ll}
\max _{\mathbf{w}_{k}, t} & t \\
\text { s.t. } & {\left[\sum_{k=1}^{K} \mathbf{w}_{k} \mathbf{w}_{k}^{H}\right]_{i, i} \leq p_{i}, \quad \forall i} \\
& \mathbf{h}_{n_{k}}^{H} \mathbf{w}_{k} \mathbf{w}_{k}^{H} \mathbf{h}_{n_{k}} \geq t \quad \forall k, \\
& \mathbf{h}_{n_{j}}^{H} \mathbf{w}_{k} \mathbf{w}_{k}^{H} \mathbf{h}_{n_{j}} \leq \varepsilon \quad \forall k \neq j .
\end{array}
$$

The value of $\varepsilon$ determines whether the problem is of the ZF type, the MRT type, or a variant thereof. When $\varepsilon$ is negligible compared to the noise power, the formulation describes a ZF-based approach, and when $\varepsilon$ is of the order of the noise power this represents a regularized ZF-based approach (cf. Spencer et al., 2004). When $\varepsilon$ is sufficiently large, the constraints in $(4.25 \mathrm{~d})$ become inactive, and accordingly the formulation describes an MRT-based approach.

One strategy for solving (4.25) is to employ a semidefinite relaxation (Luo et al., 2010). As in related beamforming methods based on semidefinite relaxation (e.g., Bengtsson and Ottersten, 2001), that approach involves the solution of a convex optimization problem for a set of matrices and a post-processing step that extracts good beamformers from these matrices. However, the computational cost of solving the convex optimization problem is even higher than that of the offset maximization algorithm, and that is only the cost of determining the beamforming directions. Accordingly, in the following sections we will present low-cost algorithms for robust beamforming with PAPCed variants of the ZF and MRT beamforming directions. 


\subsubsection{ZF beamforming with PAPCs only}

When $\varepsilon=0$, the problem in (4.25) involves finding the beamforming vectors that remove the interference at the receivers under the nominal channel conditions and satisfy the PAPCs. The essence of this problem was addressed in (Wiesel et al., 2008) using a re-parametrization technique. In particular, let us define the matrix $\mathbf{H}$

as the matrix whose $k$ th column is $\mathbf{h}_{n_{k}}$ and the matrix $\tilde{\mathbf{U}}_{\mathrm{ZF}}=\mathbf{H}\left(\mathbf{H}^{H} \mathbf{H}\right)^{-1}$. The $k$ th column of $\tilde{\mathbf{U}}_{\mathrm{ZF}}$, denoted $\tilde{\mathbf{u}}_{\mathrm{ZF}}$ is a zero-forcing direction for the $k$ th user with a unit signal gain; i.e., $\tilde{\mathbf{u}}_{\mathrm{ZF}_{k}}^{H} \mathbf{h}_{n_{k}}=1$. If we let $\mathbf{H}_{\perp}$ denote a matrix whose columns form a basis for the null space of $\mathbf{H}$, then the set of all ZF directions for the $k$ th user is given by the $k$ th column of $\tilde{\mathbf{U}}_{\mathrm{ZF}}+\mathbf{H}_{\perp} \mathbf{M}$, for an arbitrary scaling matrix $\mathbf{M}$. Accordingly, the solution to the problem in (4.25) takes the form

$$
\mathbf{w}_{k}=\sqrt{t}\left(\tilde{\mathbf{u}}_{\mathrm{ZF}_{k}}+\mathbf{H}_{\perp} \mathbf{m}_{k}\right),
$$

where $\mathbf{m}_{k}$ is the $k$ th column of matrix $\mathbf{M}$ (Wiesel et al., 2008). Note that the constraints in (4.25c) and (4.25d) (with $\varepsilon=0$ ) are automatically satisfied by designing the precoding vectors $\mathbf{w}_{k}$ in the form in (4.26). The conditions that remain to be met are the PAPCs, and that can be done by adjusting the scaling matrix M. (Wiesel et al., 2008) formulated this problem as a convex quadratically-constrained program that can be efficiently solved

$$
\begin{array}{ll}
\min _{\mathbf{M}, \hat{p}} & \hat{p} \\
\text { s.t. } & \left\|\left(\tilde{\mathbf{U}}_{\mathrm{ZF}}+\mathbf{H}_{\perp} \mathbf{M}\right)^{H} \tilde{\mathbf{e}}_{i}\right\|^{2} \leq \hat{p}, \quad \forall i,
\end{array}
$$


where $\tilde{\mathbf{e}}_{i}$ is the $i$ th column of the identity matrix. To complete the design, we choose the largest value for $t$ such that $t \hat{p} \leq p_{i}, \forall i$, which means that the beamformers of the form in (4.26) satisfy the remaining constraints; i.e., those in (4.25b).

If we let the $(k, k)$ th entry of the diagonal matrix $\hat{\mathbf{Q}}$ denote the dual variable of the $k$ th PAPC in (4.27b), then the KKT conditions of the dual problem of (4.27) show that the scaling matrix should satisfy $\mathbf{M}=-\left(\mathbf{H}_{\perp}^{H} \hat{\mathbf{Q}} \mathbf{H}_{\perp}\right)^{\dagger}\left(\mathbf{H}_{\perp}^{H} \hat{\mathbf{Q}} \tilde{\mathbf{U}}_{\mathrm{ZF}}\right)$. Although such a relation does not allow for a closed-form solution, as we do not know $\hat{\mathbf{Q}}$, it does allow for the integration of the robust power loading method in (Medra and Davidson, 2015a), as an alternative to giving all the users the same nominal signal strength $t$. Furthermore, the explicit relation between $\hat{\mathbf{Q}}$ and $\mathbf{M}$ allows us to use the sub-gradient algorithm for $\hat{\mathbf{Q}}$ and to calculate $\mathbf{M}$ accordingly. The proposed algorithm is summarized as Algorithm 4.

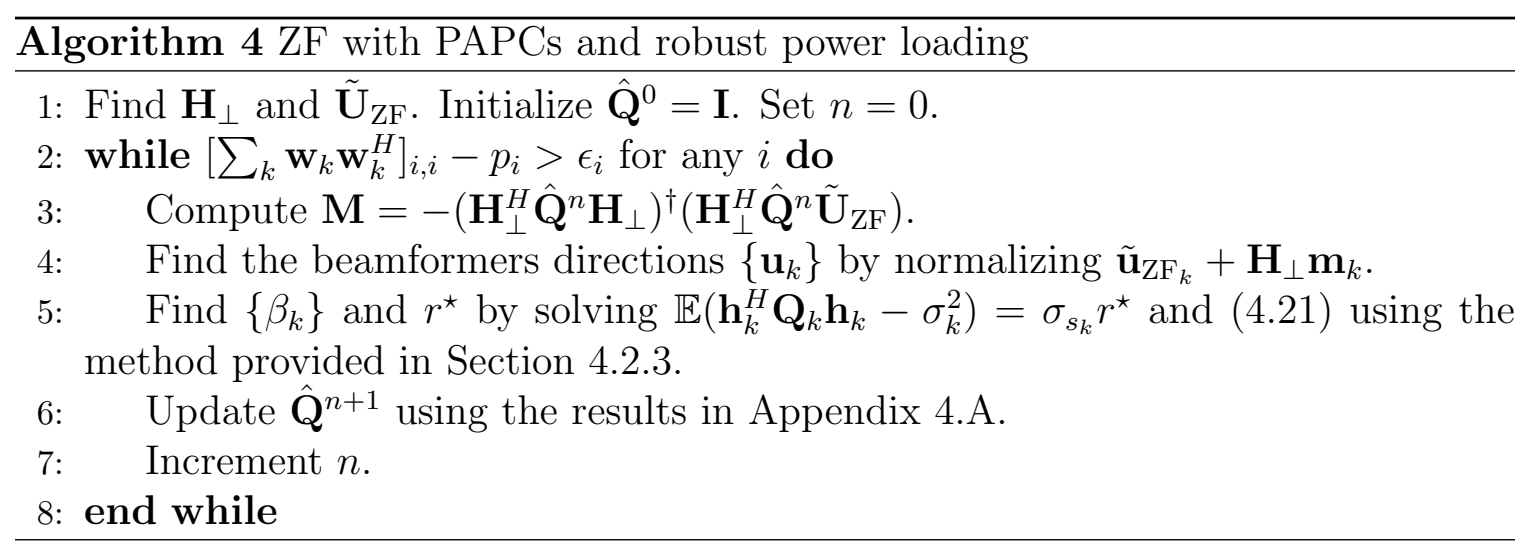

From a computational respective, the key steps in the initialization of this algorithm are the finding of the ZF directions and the null space of $\mathbf{H}$, which requires $\mathcal{O}\left(N_{t}^{2} K\right)$ operations. Each iteration of the algorithm involves the iterative solution of the $K+1$ linear equations in step 5, which, as explained in Section 4.2.3, requires $\mathcal{O}\left(K^{3}\right)$ operations, and the matrix operations required to update $\mathbf{M}$ in step 3, which 
require $\mathcal{O}\left(\left(N_{t}-K\right)^{3}\right)$ operations. When the number of antennas $N_{t}$ is close to the number of users $K$, the dimensions of the matrix $\mathbf{H}_{\perp}^{H} \hat{\mathbf{Q}} \mathbf{H}_{\perp}$ are small, which means that in that case the computational cost of this algorithm is dominated by finding the ZF directions and the null space in the initialization step.

\subsubsection{Generalized ZF beamforming}

The extension of the ZF design with PAPCs to accommodate a total power constraint is straightforward, and follows the same steps that were used in the generalized offset maximization problem; see Section 4.3.2. The generalized ZF problem can be formu-

lated by adding the total power constraint $\sum_{k=1}^{K} \mathbf{w}_{k}^{H} \mathbf{w}_{k} \leq P_{t}$ to the constraints in (4.25). Then we consider the equivalent power minimization problem, assuming, for now, that the optimal $t$ is known

$$
\begin{array}{ll}
\min _{\mathbf{M}} & t \sum_{i}\left\|\left(\tilde{\mathbf{U}}_{\mathrm{ZF}}+\mathbf{H}_{\perp} \mathbf{M}\right) \tilde{\mathbf{e}}_{i}\right\|^{2} \\
\text { s.t. } & t\left\|\left(\tilde{\mathbf{U}}_{\mathrm{ZF}}+\mathbf{H}_{\perp} \mathbf{M}\right)^{H} \tilde{\mathbf{e}}_{i}\right\|^{2} \leq p_{i}, \quad \forall i .
\end{array}
$$

Consistent with our previous analysis, we will let $\hat{\mathbf{Q}}$ denote the diagonal matrix with the dual variables of the PAPCs on its diagonal. From the KKT conditions we can then show that $\mathbf{M}=-\left(\mathbf{H}_{\perp}^{H}\left(\hat{\mathbf{Q}}+\mathbf{I}_{N_{t}}\right) \mathbf{H}_{\perp}\right)^{-1}\left(\mathbf{H}_{\perp}^{H}\left(\hat{\mathbf{Q}}+\mathbf{I}_{N_{t}}\right) \tilde{\mathbf{U}}_{\mathrm{ZF}}\right)$. Furthermore, as in the previous algorithm we replace the uniform power loading, $t$, with the robust power loading by Medra and Davidson (2015a). The resulting modified version of Algorithm 4 is stated in Algorithm 5. As is apparent from Algorithm 5, the order of its computational cost is the same as that of Algorithm 4 . 


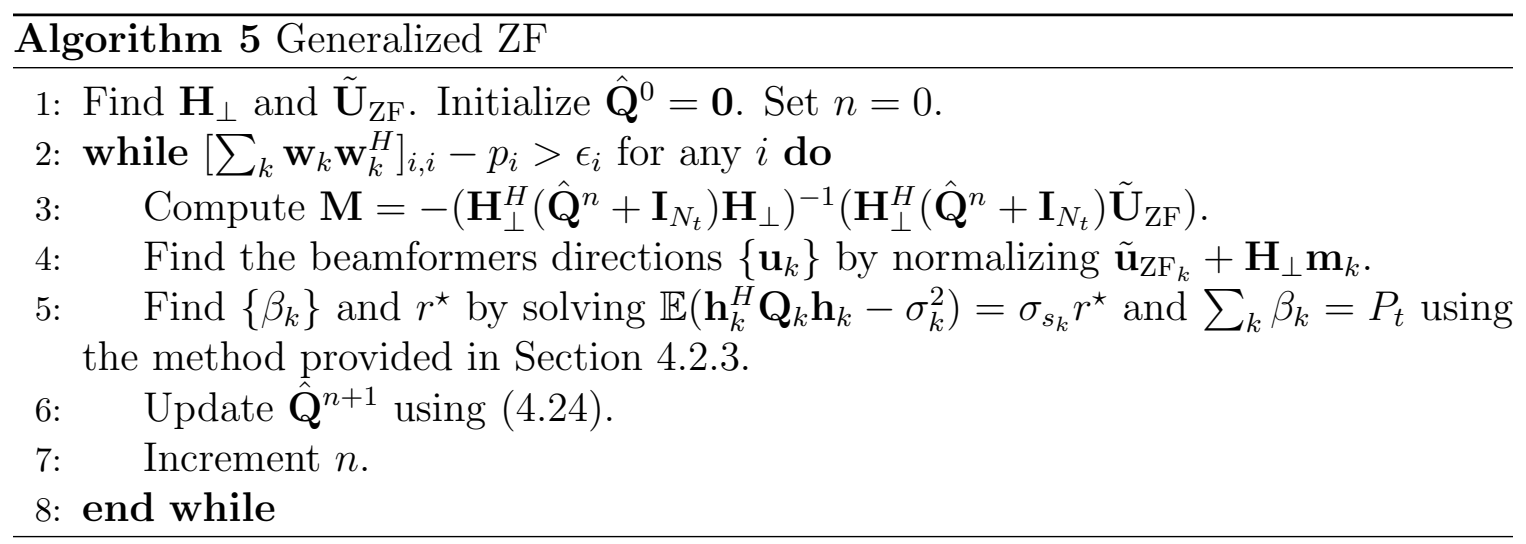

\subsection{Conventional MRT with per-antenna power con- straints}

As we have seen in the previous section, our approach to imposing PAPCs on the class of ZF beamformers can result in an algorithm of lower computational cost than that of offset maximization with PAPCs. However, any advantage is dependent on the size of the null space of the channel matrix. In settings with a large number of antennas and a small number of users, such as those arise in massive MIMO, the size of the null space can be quite large. In this section, we will show how the complexity can be further reduced by using an MRT-based approach rather than the ZF-based approach.

\subsubsection{MRT with PAPCs}

In the MRT case, the interference conditions $\mathbf{h}_{n_{j}}^{H} \mathbf{w}_{k} \mathbf{w}_{k}^{H} \mathbf{h}_{n_{j}} \leq \varepsilon$ are omitted from the problem in (4.25), and the problem of finding nominal MRT-based beamformers that 
satisfy PAPCs can be written as

$$
\begin{array}{ll}
\max _{\mathbf{w}_{k}, t} & t \\
\text { s.t. } & {\left[\sum_{k=1}^{K} \mathbf{w}_{k} \mathbf{w}_{k}^{H}\right]_{i, i} \leq p_{i}, \quad \forall i} \\
& \mathbf{h}_{n_{k}}^{H} \mathbf{w}_{k} \mathbf{w}_{k}^{H} \mathbf{h}_{n_{k}} \geq t, \quad \forall k .
\end{array}
$$

Following a similar analysis to those performed earlier, if we let $q_{i}$ denote the dual variable for the $i$ th PAPC, define the diagonal matrix $\hat{\mathbf{Q}}$ such that $[\hat{\mathbf{Q}}]_{i, i}=q_{i}$, and define $\nu_{k}$ to be the dual variable for the $k$ th condition in $(4.29 \mathrm{c})$, then the Lagrangian of the problem in (4.29) can be written as:

$$
\begin{aligned}
\mathcal{L}\left(t, \mathbf{w}_{k}, \nu_{k}, q_{i}\right)=-t+\sum_{i=1}^{N_{t}} q_{i}\left(\left[\sum_{k=1}^{K} \mathbf{w}_{k} \mathbf{w}_{k}^{H}\right]_{i, i}-p_{i}\right) & \\
& -\sum_{k=1}^{K} \nu_{k}\left(\mathbf{h}_{n_{k}}^{H} \mathbf{w}_{k} \mathbf{w}_{k}^{H} \mathbf{h}_{n_{k}}-t\right) .
\end{aligned}
$$

Accordingly, we can state the KKT conditions in a simplified form as $\sum_{k=1}^{K} \nu_{k}=1$, $t=\sum_{i=1}^{N_{t}} q_{i} p_{i}$, and $\hat{\mathbf{Q}} \mathbf{w}_{k}=\nu_{k} \mathbf{h}_{n_{k}} \mathbf{h}_{n_{k}}^{H} \mathbf{w}_{k}$. The last condition can be re-written as $\mathbf{w}_{k}=\nu_{k} \mathbf{h}_{n_{k}}^{H} \mathbf{w}_{k} \hat{\mathbf{Q}}^{-1} \mathbf{h}_{n_{k}}$, which means that $\mathbf{w}_{k}$ and $\hat{\mathbf{Q}}^{-1} \mathbf{h}_{n_{k}}$ have the same direction. We note that at optimality $[\hat{\mathbf{Q}}]_{i, i}$ is equal to the $i$ th element of $\mathbf{h}_{n_{k}}$, scaled by $\nu_{k} \mathbf{h}_{n_{k}}^{H} \mathbf{w}_{k}$, then divided by the $i$ th element of $\mathbf{w}_{k}$. This equation does not allow any optimal $[\hat{\mathbf{Q}}]_{i, i}$ to be zero except if the channel vector $\mathbf{h}_{n_{k}}$ contains a zero, which, under most reasonable channel models, is a "zero-probability" event. Since each $q_{i}$ is positive, the constraints in (4.29b) are all active, and accordingly $\sum_{k} \beta_{k}=\sum_{i} p_{i}$.

Similar to the analysis of the previous problems, if we know $\hat{\mathbf{Q}}$, then we can 
find the beamforming directions using $\hat{\mathbf{Q}}^{-1} \mathbf{h}_{n_{k}}$ and subsequently solve for the power loading using the linear equations that arise when $(4.29 \mathrm{c})$ holds with equality. The value of $t$ in $(4.29 \mathrm{c})$ can be calculated using the KKT equation $t=\sum_{i=1}^{N_{t}} q_{i} p_{i}$. If the equations in (4.29c) were not satisfied with equality at optimality, we could rescale the beamforming vectors to get a larger value of $t$, which would contradict the assumed optimality. This observation is similar to the observation in the offset maximization section that enabled the use of the subgradient algorithm to find $\hat{\mathbf{Q}}$. Accordingly, we can suggest the iterative algorithm in Algorithm 6.

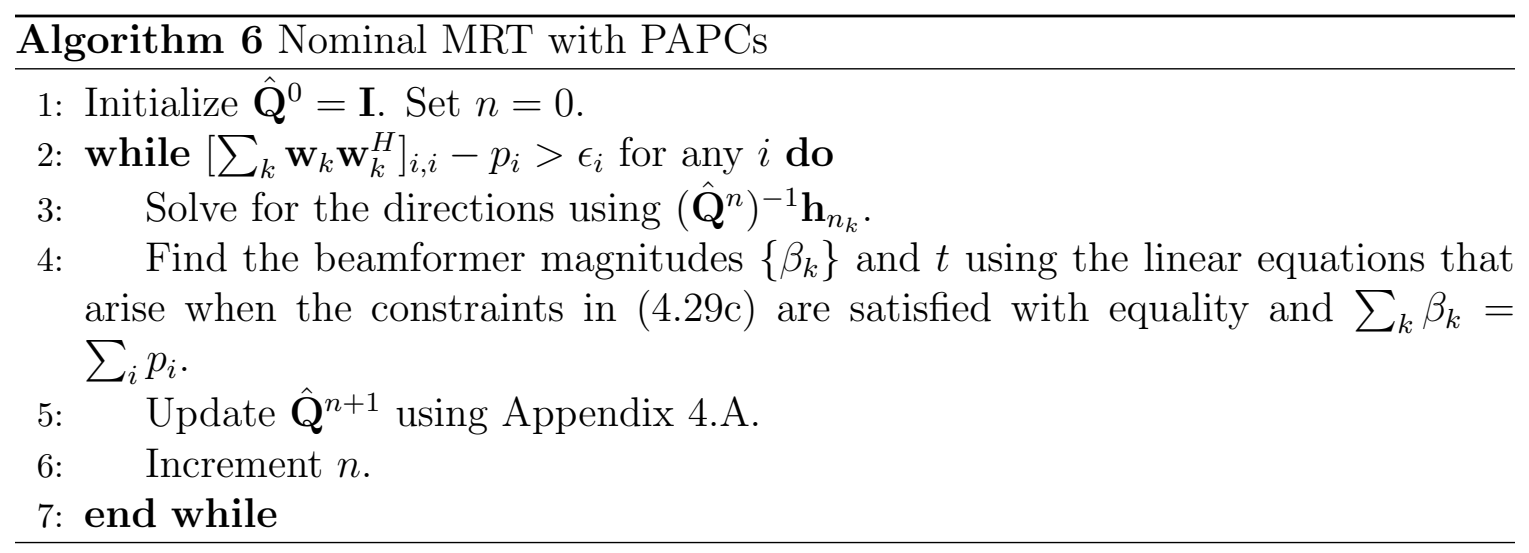

Algorithm 6 provides an iterative way to find the values of $\hat{\mathbf{Q}}$, and, accordingly, the optimal precoding vectors. Its complexity per iteration is no more than linear in $N_{t}$. However, we will now develop a closed-form expression that approximates the optimal solution of Algorithm 6 when the PAPCs are the same; i.e., $p_{i}=p, \forall i$. This closed-form removes the need for any iterations, which allows for an algorithm that is suitable for massive MIMO settings. To develop the approximation, we first note that the PAPCs and the MRT constraints hold with equality at optimality. That 
means that at optimality

$$
\begin{aligned}
{\left[\sum_{k=1}^{K} \mathbf{w}_{k} \mathbf{w}_{k}^{H}\right]_{i, i} } & =\sum_{k=1}^{K} \nu_{k}^{2}\left|\mathbf{h}_{n_{k}}^{H} \mathbf{w}_{k}\right|^{2}\left|\left[\hat{\mathbf{Q}}^{-1} \mathbf{h}_{n_{k}}\right]_{i}\right|^{2} \\
& =\sum_{k=1}^{K} \nu_{k}^{2} t\left|\left[\hat{\mathbf{Q}}^{-1} \mathbf{h}_{n_{k}}\right]_{i}\right|^{2} \\
& =p
\end{aligned}
$$

Now let us define

$$
g_{i}=\sum_{k=1}^{K} \nu_{k}^{2}\left|\left[\mathbf{h}_{n_{k}}\right]_{i}\right|^{2}
$$

Using (4.31), we can also write $g_{i}=p q_{i}^{2} / t=p q_{i}^{2} /\left(\sum_{i=1}^{N_{t}} q_{i} p\right)$. Accordingly, we can calculate $q_{i}$ from $\left\{g_{i}\right\}$ as $q_{i}=\left(\sum_{j} \sqrt{g_{j}}\right) \sqrt{g_{i}}$. The objective of maximizing $t=\sum_{i=1}^{N_{t}} q_{i} p$ is, therefore, equivalent to maximizing $\sum_{j} \sqrt{g_{j}}$. Since the dual variables $\nu_{k}^{2}$ enter (4.32) as weighting variables for the power gains of the components of $\mathbf{h}_{n_{k}}$, the optimal values of $\nu_{k}$ are influenced by the relative values of the elements of each set $\left\{\left|\left[\mathbf{h}_{n_{k}}\right]_{i}\right|^{2}\right\}_{k=1}^{K}$. When these elements have the same distribution, the optimal values of $\nu_{k}$ tend to get closer as the number of antennas grows. Since $\sum_{k} \nu_{k}=1$, that suggests the approximation $\nu_{k} \approx 1 / K$. Since the approximation only holds in the limit, there will be discrepancy between the actual power on the antennas and $p$, but as the number of antennas grows, that difference decreases. For a finite number of antennas, we may rescale the result so that the PAPCs are satisfied. That is done in steps 6 and 7 in Algorithm 7.

Both of the algorithms for the nominal MRT-based approach (Algos 6 and 7) result in beamformers that satisfy the PAPCs. As we will see in the simulation section, the resulting beamformers provide similar outage performance even for relatively small 


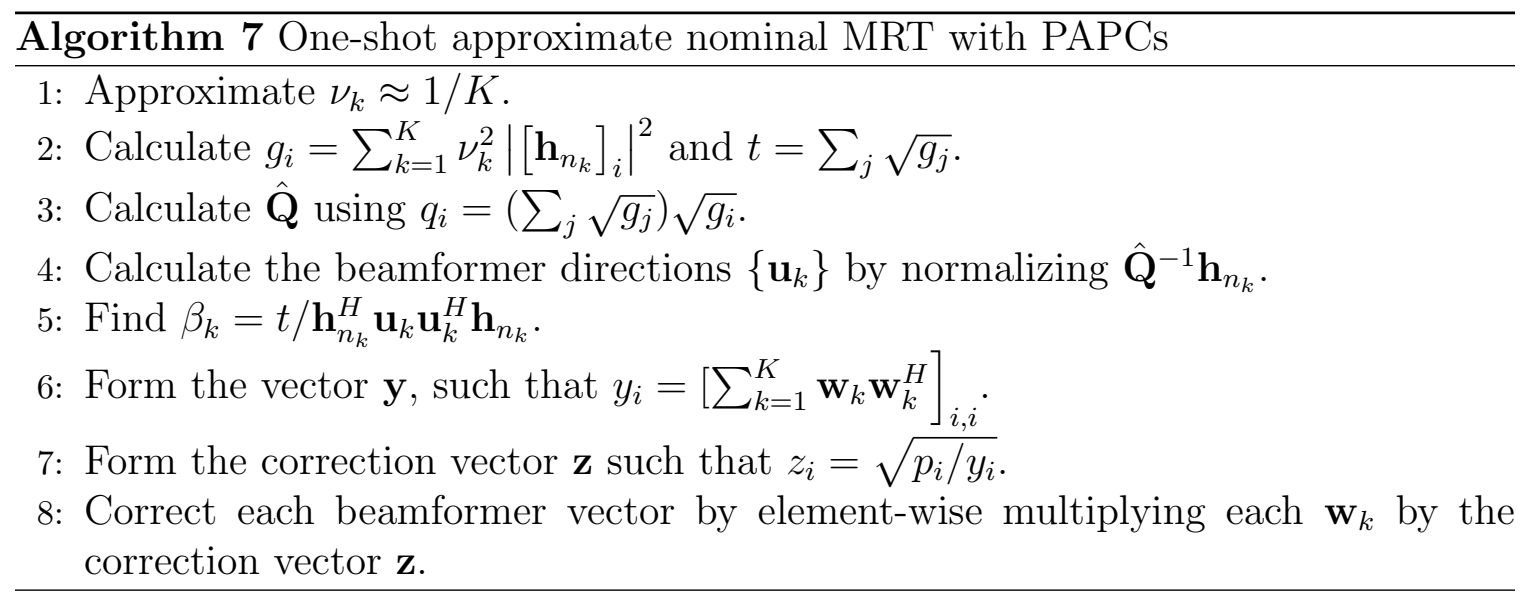

number of antennas. However, both of the algorithms are based on nominal performance criteria and any robustness that is obtained arises only implicitly. To address that point, we observe that Algorithm 6 updates $\hat{\mathbf{Q}}$ iteratively using the sub-gradient algorithm, which allows for the incorporation of the robust power loading described in Section 4.2.3. The ability to incorporate that power loading can significantly reduce the outage probability by allocating each user an appropriate amount of power rather than forcing the nominal signal power of different users to be the same value $t$. The resulting algorithm is stated in Algorithm 8. In scenarios in which it is reasonable to use the same value of $t$ for all users, or when we can pre-define different weights for the value of $t$, Algorithm 7 can provide a closed-form solution that is close to the optimal one, without the need for any iterations.

The complexity of Algorithm 8 is dominated by operations that are linear in the number of antennas for each user. This means that the complexity per iteration is of the order of $\mathcal{O}\left(N_{t} K\right)$ operations. The robust power loading can be effectively approximated in the massive MIMO settings so that it requires only $\mathcal{O}\left(N_{t} K\right)$ operations, beside the $\mathcal{O}\left(K^{3}\right)$ operations for the initial matrix inversion (Medra and Davidson, 2015a). 


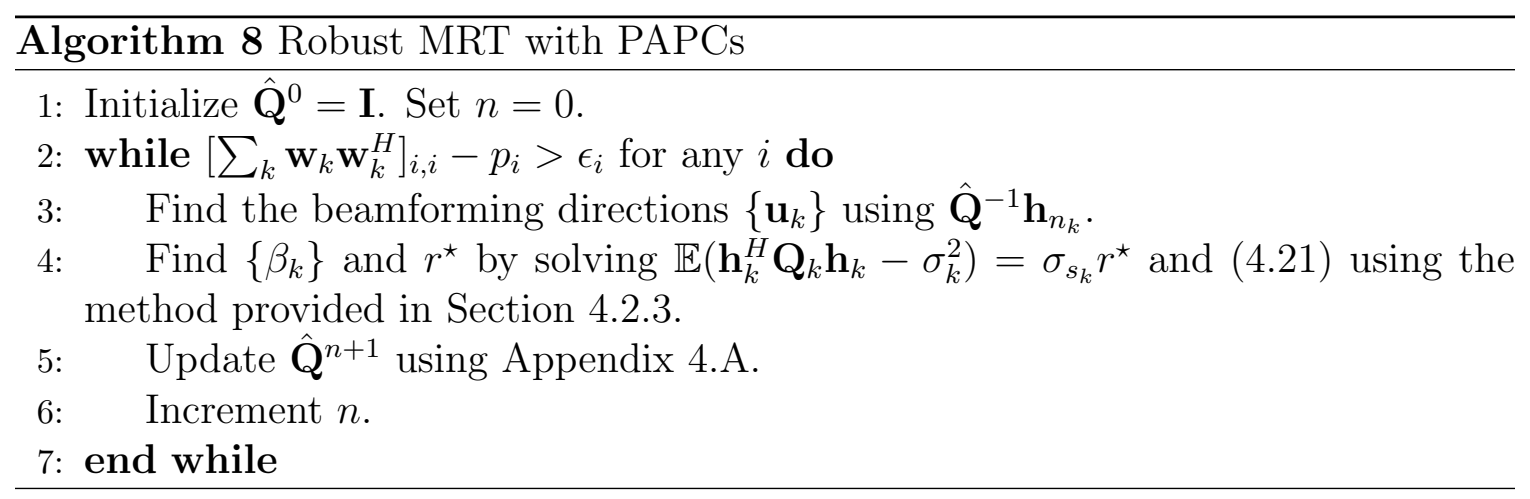

\subsubsection{Generalized MRT}

The derivation of the MRT-based algorithm when the total power constraint is added to (4.29) follows the same steps that were performed in the ZF case and the offset maximization case. The modified algorithm is presented in Algorithm 9.

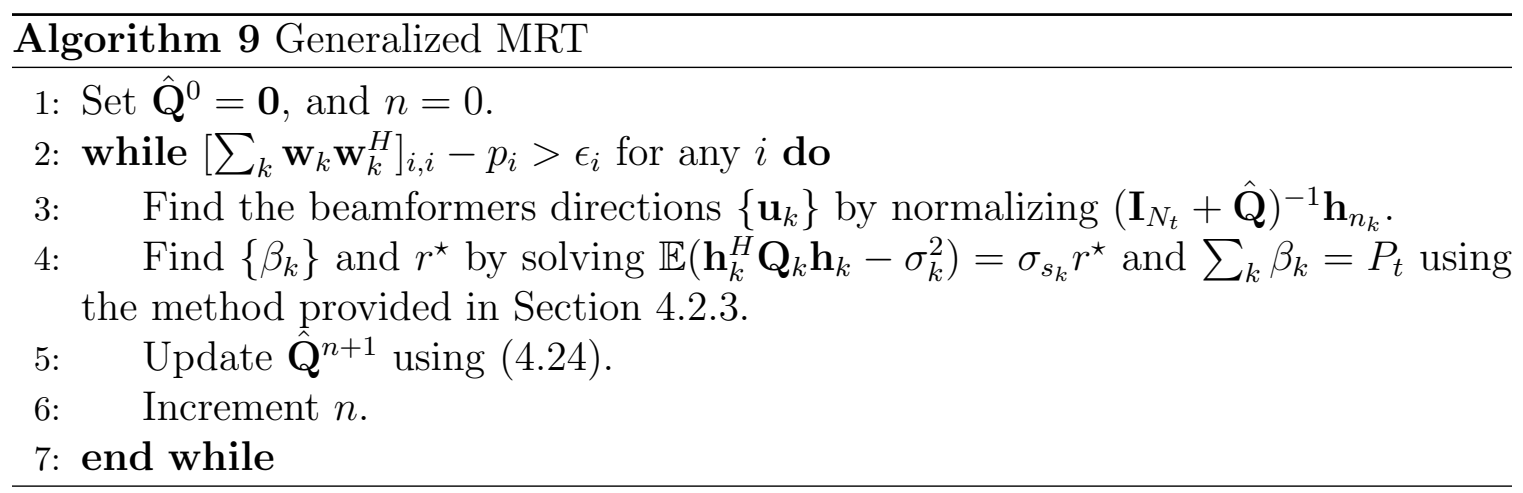

\subsection{Simulation results}

In this section, we will show how the application of PAPCs to substantially reduce the dynamic range of the power transmitted from each antenna can be implemented without significantly degrading the outage probability of the system. We consider a system in which a BS with $N_{t}$ antennas serves $K$ single-antenna users distributed 
uniformly in a disk of radius $3.2 \mathrm{~km}$ around the BS. The large scale fading is modelled using a path-loss exponent of 3.52 and log-normal shadow fading with $8 \mathrm{~dB}$ standard deviation. The small scale fading is modelled using the standard i.i.d. Rayleigh model. We assume an additive channel estimation error of covariance 0.04I, and an SINR target of $\gamma=3 \mathrm{~dB}$ for all users. For the algorithms with PAPCs only, the PAPC is uniform and is set to $p_{i}=P_{t} / N_{t}$, where $P_{t}$ is the total power constraint, which is implicit in this case. For the generalized algorithms with both PAPCs and a total power constraint (Algos 3, 5, and 9), the PAPCs are set to be slightly larger, so that the total power constraint is active. For these cases we choose $p_{i}=1.2 P_{t} / N_{t}$. We assume that each user has a signal sensitivity of $-90 \mathrm{dBm}$, and we will consider this power as the noise power. The termination parameter for the algorithms is chosen to be $\epsilon_{i}=0.1 p_{i}$, and each experiment is repeated on 20,000 channel realizations. A simple channel-strength user selection technique is employed, where users having $\left\|\mathbf{h}_{e_{k}}\right\|^{2} P_{t} / k \sigma_{k}^{2} \geq \gamma_{k}$ are served.

To demonstrate the application of PAPCs with offset maximization, in Fig. 4.1 we plot the outage probability versus the total power constraint $P_{t}$ for six different algorithms in a scenario in which $N_{t}=4$ and $K=3$. The first algorithm is the nominal PAPCed design algorithm presented by Yu and Lan (2007), with the beamforming vectors scaled so that the total power is equal to $P_{t}$. This is equivalent to solving (4.17) when $r_{p a}^{\star}=0$, then scaling the resulting beamforming vectors. We compare the performance of Yu and Lan (2007) to the performance of Algo. 1 with and without the acceleration step, and Algo. 2 with the acceleration step. We note that while the performance of Algo. 1 is close to that of Yu and Lan (2007), the application of the robust power loading in Algo. 2 provides a significant reduction 
in the outage probability. To assess the impact of the PAPCs we compare the performance of Algo. 2 to that of the robust offset maximization technique with a total power constraint only (Medra and Davidson, 2015a). As seen in Fig. 4.1, Algo. 2 achieves a performance close to that of Medra and Davidson (2015a) even though it imposes PAPCs. As expected, the performance of Algo. 3, which imposes a total power constraint and weaker PAPCs, falls in between that of Medra and Davidson (2015a) and Algo. 2.

The convergence rate of the subgradient algorithm strongly depends on how the step size is chosen and, hence, this should be tailored to the application. Based on insights from (Scutari et al., 2014) we have chosen a step size that is updated using $t_{n}=t_{n-1}-t_{n-1}^{2} / 1000$. Our numerical experience has suggested choosing $t_{0}=$ $N_{t} /\left(P_{t} K\right)$. To examine the potential impact of the prediction scheme outlined in Section 4.3.3, we have implemented a linear predictor of the form $\hat{\mathbf{Q}}_{p}^{1}=2.8 \operatorname{diag}\left(\mathbf{q}^{1}\right)$ 1.8I. To show the effectiveness of these choices, we plot in Fig. 4.2 the percentage of violated PAPCs versus the iteration number for the scenario in which $P_{t}=40$. We set the violation to one when any antenna is transmitting a power that is more than $10 \%$ higher than $p_{i}$. (Recall that we set $\epsilon_{i}=0.1 p_{i}$.) We observe from Fig. 4.2 that within the first few iterations, the PAPCs are met in most cases. We also note that the acceleration step can reduce the average number of iterations while providing almost the same outage performance. In order to provide context for these results, we point out that the average number of iterations required by the nominal algorithm by $\mathrm{Yu}$ and Lan (2007) is much higher. Indeed, as shown by Yu and Lan (2007), it can range from a few tens to hundreds in analogous settings.

In assesing the performance of the ZF-based PAPCed beamforming algorithms, 


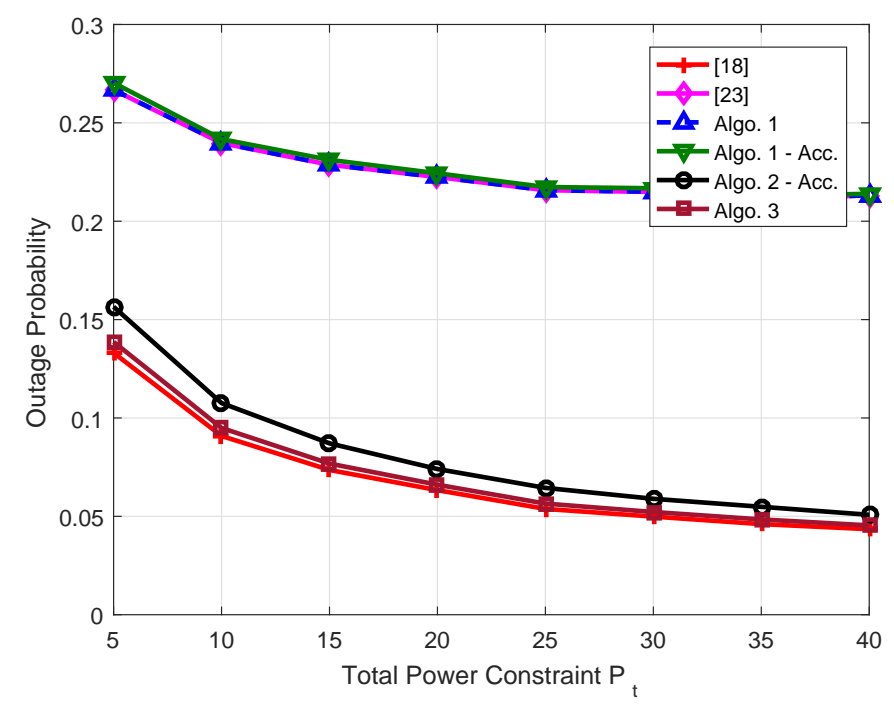

Figure 4.1: Outage probability for a 4 antenna BS serving 3 users with a total transmitted power of $P_{t}$. Here [18] refers to Medra and Davidson (2015a), and [23] refers to $\mathrm{Yu}$ and Lan (2007).

rather than examining the outage performance against the transmission power, we will fix the total power constraint to $P_{t}=2$ and examine the performance as the number of antennas, $N_{t}$, increases. Other than that, the scenario is the same as the previous one. As performance benchmarks for Algo. 4, we have included the performance of the algorithm by Wiesel et al. (2008) which maximizes the minimum received signal power, $\mathbf{h}_{e_{k}}^{H} \mathbf{w}_{k} \mathbf{w}_{k}^{H} \mathbf{h}_{e_{k}}$, and a modified version of the algorithm by Wiesel et al. (2008) that maximizes the minimum value of $\mathbf{h}_{n_{k}}^{H} \mathbf{w}_{k} \mathbf{w}_{k}^{H} \mathbf{h}_{n_{k}}$ instead. We observe that in the case of noisy channel estimates, the normalization step significantly reduces the outage probability. More importantly, the application of the robust power loading in Algo. 4 provides significantly better performance. As a lower bound on the outage achieved by Algo. 4 we consider ZF beamforming with the nominal ZF directions and robust power loading with only a total power constraint (Medra and Davidson, 2015a); i.e., 


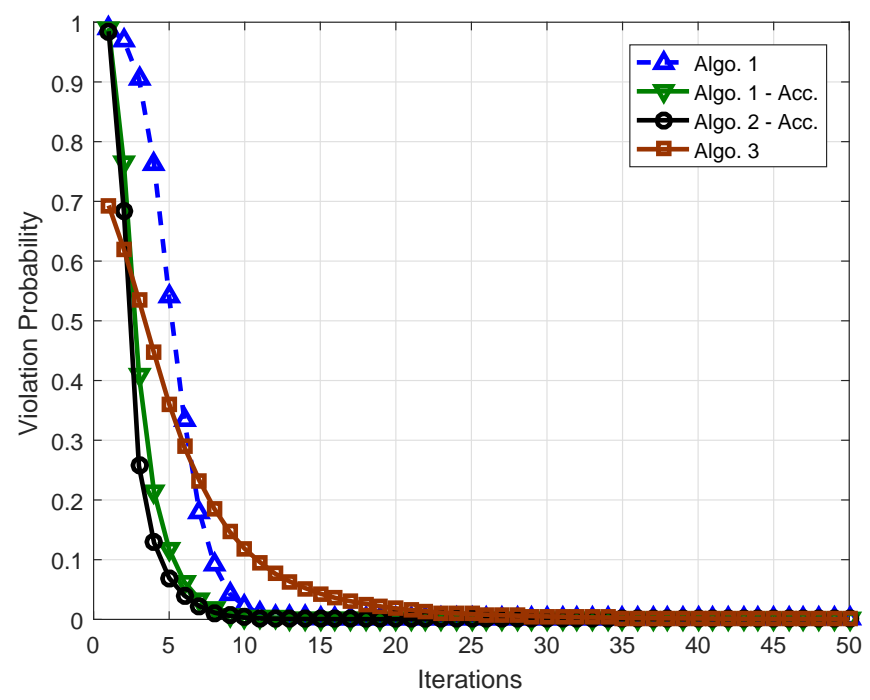

Figure 4.2: Convergence behaviour for a 4 antenna BS serving 3 users with a total transmitted power of $P_{t}$. The violation probability measures the fraction of the 20,000 realizations for which at least one PAPC was violated by more then $10 \%$ at the given iteration of the algorithm. 


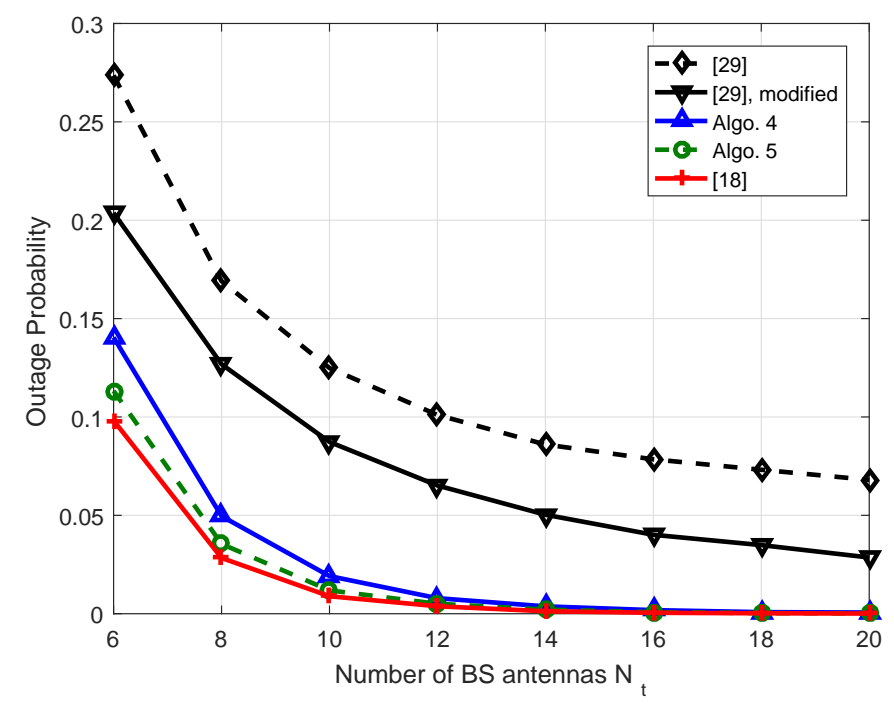

Figure 4.3: Outage probability for a BS serving 3 users with a total transmitted power of $P_{t}=2$. Here [18] refers to Medra and Davidson (2015a), and [29] refers to Wiesel et al. (2008).

without the PAPCs. The resulting comparison shows that the degradation incurred by imposing the PAPCs is quite small. Finally, as expected, the performance of the generalized algorithm (Algo. 5) lies in between that of Algo. 4 and that by Medra and Davidson (2015a).

To assess the performance of the MRT-based PAPCed algorithms, we will allow for more users, $K=8$, and set the total power constraint $P_{t}$ to be 1 . As in the $\mathrm{ZF}$ case, we examine the outage performance versus the total number of antennas, $N_{t}$, but we do so for a larger number of antennas. In Fig. 4.4, the performance of Algos 6, 7, and 8 is compared to the performance of the algorithm by Feng and Jing (2016). We observe that the performance of Algos 6, and 7 is almost identical to that of the algorithm presented by Feng and Jing (2016), and that the performance of Algo. 8 is superior. As a benchmark, the performance of the robust MRT beamformer with 


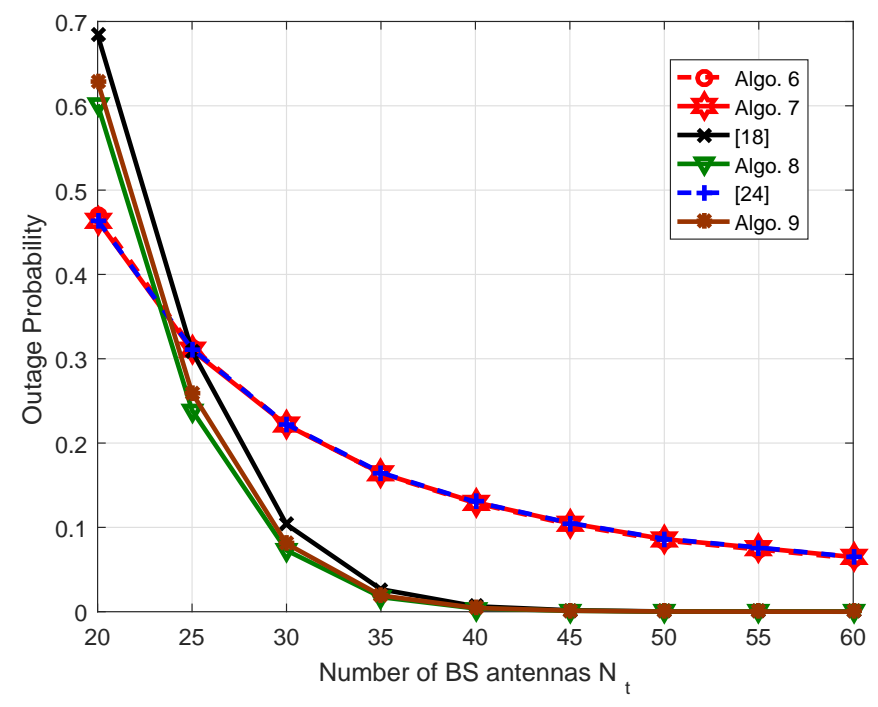

Figure 4.4: Outage probability for a BS serving 8 users with a total transmitted power of $P_{t}=1$. Here [18] refers to Medra and Davidson (2015a), and [24] refers to Feng and Jing (2016).

only a total power constraint (i.e., no PAPCs, Medra and Davidson, 2015a) is plotted in Fig. 4.4. The performance of the generalized algorithm (Algo. 9) is also plotted.

\subsection{Conclusion}

In this paper, we developed low-complexity algorithms for finding robust beamformers that provide low outage of target SINRs while satisfying specified per-antenna power constraints (PAPCs). Initially, we used insights from the subgradient method for designing PAPCed beamformers in the case of perfect channel state information (Yu and Lan, 2007) to obtain PAPCed version of the offset maximization algorithm developed by Medra et al. (2016). Further reductions in the outage probability were then obtained by incorporating the robust power loading presented by Medra and 
Davidson (2015a) into the design problem. While the proposed algorithms are of low complexity, we identified the evaluation of the beamforming directions as the computational bottleneck. To address that, we developed algorithms that employ PAPCed variants of the conventional zero-forcing (ZF) and maximum ratio transmission (MRT) directions and incorporate the robust power loading. In the process of doing so, we developed a closed-form expression for an MRT-based beamformer that satisfies PAPCs and may be appropriate for massive MIMO systems. Our simulation results revealed that PAPCed beamforming can be achieved without incurring a significant degradation in outage performance.

\section{A Appendix: $\hat{Q}$ update}

To determine the updated value for $\hat{\mathbf{Q}}^{n+1}$, we have to determine the projection,

$$
\hat{\mathbf{Q}}^{n+1}=\operatorname{proj}\left(\hat{\mathbf{Q}}^{n}+t_{n} \operatorname{diag}\left(\operatorname{diag}\left(\sum_{i} \mathbf{w}_{i} \mathbf{w}_{i}^{H}\right)\right)\right) .
$$

To do so, we let $\mathbf{q}=\operatorname{diag}\left(\hat{\mathbf{Q}}^{n+1}\right)$, and $\mathbf{q}_{o}=\operatorname{diag}\left(\hat{\mathbf{Q}}^{n}+t_{n} \operatorname{diag}\left(\operatorname{diag}\left(\sum_{i} \mathbf{w}_{i} \mathbf{w}_{i}^{H}\right)\right)\right)$. That enables us to write the projection problem as

$$
\begin{array}{ll}
\min _{\mathbf{q}} & \left\|\mathbf{q}-\mathbf{q}_{o}\right\|^{2} \\
\text { s.t. } & \sum_{i=1}^{N_{t}} q_{i} p_{i}=\sum_{i=1}^{N_{t}} p_{i} \\
& q_{i} \geq 0, \quad \forall i .
\end{array}
$$


If we let $\zeta$ denote the dual variable of the equality constraint, then from the KKT conditions of (4.33) we can show that the optimal $q_{i}$ is

$$
q_{i}=\max \left(q_{o_{i}}-p_{i} \zeta / 2,0\right)
$$

where $\zeta / 2=\left(\sum_{i, \forall q_{i} \neq 0} p_{i} q_{o_{i}}-\sum_{i} p_{i}\right) / \sum_{i, \forall q_{i} \neq 0} p_{i}^{2}$. Given the nature of dependence of $\left\{q_{i}\right\}$ and $\zeta$ on each other, we will solve for their values using a fixed-point approach. First, we initialize $\zeta=0$, and then we iteratively calculate $q_{i}$ and $\zeta$ from the provided equations until their values stabilize.

In the case of equal $p_{i}$ (i.e., $p_{i}=p, \forall i$ ), and when all the $q_{i}$ are positive (i.e., all the PAPCS are active), the update equation can be simplified to

$$
\hat{\mathbf{Q}}^{n+1}=\hat{\mathbf{Q}}^{n}+t_{n} \operatorname{diag}\left(\operatorname{diag}\left(\sum_{i} \mathbf{w}_{i} \mathbf{w}_{i}^{H}-p \mathbf{I}\right)\right) .
$$




\title{
Chapter 5
}

\section{Low-Complexity Robust Multi-Cell MISO Downlink Precoder Design}

\begin{abstract}
This paper develops low-complexity design techniques for robust linear precoders suitable for various multi-cell multiple-input single-output (MISO) downlink systems. The goal is to satisfy prespecified SINR requirements for users in multiple cells under some base station power constraints, in the absence of perfect channel state information (CSI). First, we consider the case of full cooperation between base stations and derive a simple iterative algorithm that achieves the required SINRs with high probability despite the presence of channel uncertainties. Then we consider the case of distributed coordination between base stations and develop a simple iterative algorithm that requires only very limited communication among the base stations. Our simulation results demonstrate that substantial robustness can be obtained at a low computational cost.
\end{abstract}




\subsection{Introduction}

Managing the interference among clusters of cells has the potential to provide significant performance gains over wireless networks that avoid interference between cells (Gesbert et al., 2010; Hong and Luo, 2014). One taxonomy of interference management schemes classifies them as being centralized or distributed, depending on where the design decisions are made, and as being cooperative or coordinating. In cooperative schemes multiple base stations (BSs) work together to transmit the same information to a receiver, whereas in coordinating schemes each receiver is assigned to a single BS, but the design of the transmissions from each BS is coordinated with that of the other BSs.

Among the many scenarios that could benefit from cooperation or coordination, we will focus on the multiple-input single-output (MISO) downlink case in which a cluster of BSs seek to cooperate or coordinate in the transmission of messages to multiple receivers each with a single antenna. We will focus on linear transmission schemes and hence the design variables are the shape of the beam transmitted to each receiver and the power allocated to that beam. Based on the assumption that sufficiently accurate channel state information (CSI) can be made available at the design nodes, a number of techniques for multi-cell downlink beamforming have been developed (e.g., Dahrouj and Yu, 2010; Tolli et al., 2011; Pennanen et al., 2011; Hong and Luo, 2014; Mirza et al., 2015; Qiu et al., 2010). More recently, efforts have been made to mitigate the sensitivity of those techniques to the uncertainties in the CSI that inevitably arise from the estimation and transmission of CSI (e.g., Shen et al., 2012b; Tajer et al., 2011; Pennanen et al., 2014; Vucic and Boche, 2007; Zhang et al., 2015; Shen et al., 2012a; Tshangini and Nakhai, 2013). However, many 
such techniques require the solution of optimization problems that incur significantly greater computational cost than that of the original designs for the perfect CSI case (some of which are, themselves, quite computationally costly).

In this paper, we will develop low-complexity iterative algorithms for the beamforming directions and the power loading in both a centrally-design cooperative mutlicell MISO downlink, and for a coordinated multi-cell MISO downlink that is designed in a distributed manner. Using insights from recent work on the isolated single-cell MISO downlink (Medra et al., 2015; Medra and Davidson, 2015b,a), we develop designs that provide substantial robustness to uncertainties and can be obtained using simple iterative algorithms. In our simulation experiments, these straightforward designs provide significantly lower outage rates than existing designs.

\subsection{System Model}

The system that we consider consists of a total of $K$-users each with a single antenna served by a cluster of $B$ BSs. User $k$ is served by a subset of the BSs, $\mathcal{B}_{k}$, where $\mathcal{B}_{k}$ consists of only one BS index in the case of distributed precoding and contains all the BS indices in the case of full cooperation. We assume that each BS is equipped with $N_{t}$ antennas and is provided with an imperfect version of the CSI of the users. We use the notation $\mathbf{h}_{k}^{j} \in \mathbb{C}^{N_{t}}$ to denote the channel between the BS $j$ and user $k$. we let $\mathbf{w}_{k}^{j}$ denote the designed precoding vector for transmission from BS $j$ to user $k$, and let $s_{k}$ denote the intended normalized data symbol for that user. To unify the notations

for the full cooperation and distributed cases, we define $\mathbf{h}_{k}=\left[\mathbf{h}_{k}^{1^{T}}, \mathbf{h}_{k}^{2^{T}}, \cdots, \mathbf{h}_{k}^{B^{T}}\right]^{T}$ as the stacking of all the channel vectors to user $k$, and we define $\mathbf{w}_{k}$ analogously. We also let $\sqrt{\beta_{k}}$ denote the Euclidean norm of $\mathbf{w}_{k}$ and $\mathbf{u}_{k}$ denote its normalized direction; 
i.e., $\mathbf{w}_{k}=\sqrt{\beta_{k}} \mathbf{u}_{k}$. With that notation, we can write the received signal of user $k$ in the following simplified form:

$$
y_{k}=\mathbf{h}_{k}^{H} \mathbf{w}_{k} s_{k}+\sum_{i \neq k} \mathbf{h}_{k}^{H} \mathbf{w}_{i} s_{i}+n_{k},
$$

where $n_{k}$ is the zero mean circular Gaussian noise of variance $\sigma_{k}^{2}$ at user $k$. We will translate each user's quality-of-service (QoS) constraint into an SINR requirement $\operatorname{SINR}_{k} \geq \gamma_{k}$. By defining $\mathbf{W}_{k}=\mathbf{w}_{k} \mathbf{w}_{k}^{H}$, we can write the SINR expression for user $k$ as

$$
\operatorname{SINR}_{k}=\frac{\mathbf{h}_{k}^{H} \mathbf{W}_{k} \mathbf{h}_{k}}{\mathbf{h}_{k}^{H}\left(\sum_{i \neq k} \mathbf{W}_{i}\right) \mathbf{h}_{k}+\sigma_{k}^{2}} .
$$

For a BS or a cluster management centre to calculate the SINR at each user, it needs to know the actual channel vectors $\left\{\mathbf{h}_{k}\right\}$. However, in practice only an estimate of those vectors will be available. In this paper we will model the uncertainty additively; i.e., $\mathbf{h}_{k}^{j}=\mathbf{h}_{e_{k}}^{j}+\mathbf{e}_{k}^{j}$, and with $\mathbf{e}_{k}^{j}$ being an independent zero-mean circular Gaussian random variable of covariance $\left(\sigma_{e_{k}}^{j}\right)^{2} \mathbf{I}$. Among a number of scenarios, this model is appropriate in certain time division duplexing (TDD) systems in which channels are estimated during the uplink training phase.

In the following sections, we will first review an existing approach to precoding for intra-cell interference mitigation in an isolated cell. We will then show how that approach can be integrated with inter-cell interference mitigation techniques that capture different levels of coordinations among the BSs. 


\subsection{Offset-maximization for the single cell case}

In the case of the downlink of an isolated cell, the task of satisfying the users' QoS constraints involves managing the interference imposed on one user due to simultaneous transmissions to other users in the same cell (i.e., managing intracell interference), and doing so in the presence of uncertainty in the BS's estimates of the channels to the users. Among many possible approaches to this problem, (including Zheng et al., 2008; Shenouda and Davidson, 2007, 2008b; Wang et al., 2014), we will review the offset maximization approach to robust precoding, which was initially developed by Medra et al. (2015), was extended to the case of per-antenna power constraints by Medra and Davidson (2015b) and enhanced with an alternative power loading by Medra and Davidson (2015a). A key advantage of that approach and its enhanced power loading is its low computational cost.

For the single cell case, the offset maximization approach is based on the observation that under our additive model for the uncertainty in the CSI, the constraint $\operatorname{SINR}_{k}>\gamma_{k}$ can be rewritten as

$$
\mathbf{h}_{e_{k}}^{H} \mathbf{Q}_{k} \mathbf{h}_{e_{k}}-\sigma_{k}^{2}+\mathbf{h}_{e_{k}}^{H} \mathbf{Q}_{k} \mathbf{e}_{k}+\mathbf{e}_{k}^{H} \mathbf{Q}_{k} \mathbf{h}_{e_{k}}+\mathbf{e}_{k}^{H} \mathbf{Q}_{k} \mathbf{e}_{k} \geq 0
$$

where $\mathbf{Q}_{k}=\mathbf{W}_{k} / \gamma_{k}-\sum_{j \neq k} \mathbf{W}_{j}$. This expression suggests that if we were to maximize the deterministic "offset" term in (5.3), we would obtain robustness against the terms that involve the error in the CSI. If we do so, with a total power constraint $P_{t}$, the 
semidefinite relaxation of the problem can be written as

$$
\begin{array}{rl}
r^{\star}=\max _{\mathbf{W}_{k}, r} & r \\
\text { s.t. } & \sum_{k=1}^{K} \operatorname{tr}\left(\mathbf{W}_{k}\right) \leq P_{t}, \\
& \mathbf{h}_{e_{k}}^{H} \mathbf{Q}_{k} \mathbf{h}_{e_{k}}-\sigma_{k}^{2}-r \geq 0, \\
& \mathbf{W}_{k} \succeq \mathbf{0}, \quad k=\{1,2, \ldots, K\},
\end{array}
$$

Although the problem in (5.4) is a semidefinite program and can be solved in a polynomial time, a closed-form solution was obtained by Medra et al. (2015). That solution also demonstrates that the semidefinite relaxation that led to (5.4) is tight. The derivation of the closed-form solution by Medra et al. (2015) is based on the following problem

$$
\begin{array}{ll}
\min _{\mathbf{W}_{k}} & \sum_{k=1}^{K} \operatorname{tr}\left(\mathbf{W}_{k}\right) \\
& \mathbf{h}_{e_{k}}^{H} \mathbf{Q}_{k} \mathbf{h}_{e_{k}}-\sigma_{k}^{2}-r^{\star} \geq 0, \\
& \mathbf{W}_{k} \succeq \mathbf{0}, \quad k=\{1,2, \ldots, K\},
\end{array}
$$

where $r^{\star}$ is the optimal value of (5.4). Problems (5.4) and (5.5) are equivalent in the sense that the optimal value for (5.5) is $P_{t}$ and that an optimal solution set $\left\{\mathbf{W}_{k}\right\}$ for one is also optimal for the other. The key to the derivation of the closed form is the similarity in structure between (5.5) and the power minimization problem for the perfect CSI case (Bengtsson and Ottersten, 2001; Wiesel et al., 2006; Bjornson et al., 2014). In particular, we first solve the following fixed point equations for the dual 
variables for the constraints in $(5.5 \mathrm{~b})$

$$
\nu_{k}^{-1}=\mathbf{h}_{e_{k}}^{H}\left(\mathbf{I}+\sum_{j} \nu_{j} \mathbf{h}_{e_{j}} \mathbf{h}_{e_{j}}^{H}\right)^{-1} \mathbf{h}_{e_{k}}\left(1+1 / \gamma_{k}\right) .
$$

Then we can find the beamforming directions using the eigen equation

$$
\mathbf{u}_{k}=\left(\left(\nu_{k} / \gamma_{k}\right) \mathbf{h}_{e_{k}} \mathbf{h}_{e_{k}}^{H}-\sum_{j \neq k} \nu_{j} \mathbf{h}_{e_{j}} \mathbf{h}_{e_{j}}^{H}\right) \mathbf{u}_{k} .
$$

Having found those directions, we then determine the power loading. The original power loading method by Medra et al. (2015) is based on solving the linear equations that arise from the fact that at optimality the constraints in $(5.5 \mathrm{~b})$ hold with equality and that the optimal objective value is $P_{t}$; i.e., $\sum_{i} \beta_{i}=P_{t}$. This results in $K+1$ linear equations for $\left\{\beta_{k}\right\}_{k=1}^{K}$ and $r^{\star}$. That method gives the same "robustness" $r$ to all users.

The enhanced power loading method by Medra and Davidson (2015a) is based on providing greater robustness to "weaker" users, and accordingly having comparable outage probabilities for all users. The notion of weakness is measured using the variance, $\sigma_{s_{k}}^{2}$, of $\mathbf{h}_{k}^{H} \mathbf{Q}_{k} \mathbf{h}_{k}-\sigma_{k}^{2}$. The users with higher $\sigma_{s_{k}}$ should be provided with more robustness, or offset, than other users with lower $\sigma_{s_{k}}$. Accordingly, the algorithm proposed by Medra and Davidson (2015a) is based on finding $\left\{\beta_{k}\right\}$ and $r^{\star}$ such that $\mathbb{E}\left(\mathbf{h}_{k}^{H} \mathbf{Q}_{k} \mathbf{h}_{k}-\sigma_{k}^{2}\right)=\sigma_{s_{k}} r^{\star}$, and $\sum_{k} \beta_{k}=P_{t}$. With such a strategy, the power loading algorithm allocates power such that the mean value of the rearranged SINR expression in (5.3) is proportional to its standard deviation. Although $\mathbb{E}\left(\mathbf{h}_{k}^{H} \mathbf{Q}_{k} \mathbf{h}_{k}-\sigma_{k}^{2}\right)$ is linear in each $\beta_{k}, \sigma_{s_{k}}$ is not, and thus complicates the problem. Medra and Davidson (2015a) adopted an iterative linearization technique in which $\sigma_{s_{k}}$ was determined 
from the values of $\left\{\beta_{k}\right\}_{k=1}^{K}$ and $r^{\star}$ at the previous iteration. That algorithm converges with high probability (Medra and Davidson, 2015a). The resulting power allocation provides similar outage performance for each user, and in the numerical experiments by Medra and Davidson (2015a) it provided improved overall outage performance.

\subsection{Network MIMO offset-maximization}

In the case of full cooperation, the BSs are all connected to a central processing unit, and all the CSI and the users' data are shared. In such a case, the system resembles a single BS with many distributed antennas and can be treated as a single cell but with different power constraints. Here we will consider the total power constraint and per-BS power constraints. If we define $\boldsymbol{\Lambda}_{i}$ to be a diagonal matrix with ones on the elements corresponding to the antennas of the $i$ th BS and zeros elsewhere and $P_{i}$ to be the power constraint on the $i$ th BS, then the offset maximization precoding problem can be stated as

$$
\begin{array}{rl}
\max _{\mathbf{W}_{k}, r} & r \\
\text { s.t. } & \sum_{k=1}^{K} \mathbf{w}_{k}^{H} \mathbf{\Lambda}_{i} \mathbf{w}_{k} \leq P_{i}, \quad i=\{1,2, \ldots, B\}, \\
& \sum_{k=1}^{K} \operatorname{tr}\left(\mathbf{W}_{k}\right) \leq P_{t}, \\
& \mathbf{h}_{e_{k}}^{H} \mathbf{Q}_{k} \mathbf{h}_{e_{k}}-\sigma_{k}^{2}-r \geq 0, \quad k=\{1,2, \ldots, K\} .
\end{array}
$$

This problem can be solved with generic convex optimization techniques. Although those techniques are effective in that they produce an optimal solution in polynomial time, the computational cost can still be quite high. In the following sections we 
consider two special cases of the problem in (5.8) in which we can develop tailored algorithms to solve the problem more efficiently. The first special case arises when $P_{t}<P_{i}, \forall i$, in which case the condition in (5.8b) can never be active and can be removed. The second special case arises when $P_{t}>\sum_{i} P_{i}$, in which case the condition in $(5.8 \mathrm{c})$ cannot be active.

\subsubsection{Dominant total power constraint}

In the absence of (5.8b), the problem in (5.8) is in the same form as (5.4) and hence the existing techniques can be applied directly. The difference between the single-cell case and the multi-cell case lies in the fact that the channel vector $\mathbf{h}_{k}$ is the stacking of all the channel vectors from all the BSs to a certain user $k$, and, accordingly, those channel vectors have different error vectors with different error variances. In this case, we can derive the mean and variance of $\mathbf{h}_{k}^{H} \mathbf{Q}_{k} \mathbf{h}_{k}-\sigma_{k}^{2}$ as

$$
\begin{aligned}
& \mathbb{E}\left(\mathbf{h}_{k}^{H} \mathbf{Q}_{k} \mathbf{h}_{k}-\sigma_{k}^{2}\right) \\
& =\mathbf{h}_{e_{k}}^{H} \mathbf{Q}_{k} \mathbf{h}_{e_{k}}-\sigma_{k}^{2}+\beta_{k} \mathbf{u}_{k}^{H} \boldsymbol{\Gamma}_{e} \mathbf{u}_{k} / \gamma_{k}-\sum_{j \neq k} \beta_{j} \mathbf{u}_{j}^{H} \boldsymbol{\Gamma}_{e} \mathbf{u}_{j} . \\
& \sigma_{s_{k}}^{2}=\operatorname{var}\left(\mathbf{h}_{e_{k}}^{H} \mathbf{Q}_{k} \mathbf{h}_{e_{k}}+2 \operatorname{Re}\left(\mathbf{e}_{k}^{H} \mathbf{Q}_{k} \mathbf{h}_{e_{k}}\right)+\mathbf{e}_{k}^{H} \mathbf{Q}_{k} \mathbf{e}_{k}-\sigma_{k}^{2}\right) \\
& =2 \mathbf{h}_{e_{k}}^{H} \mathbf{Q}_{k} \boldsymbol{\Gamma}_{e} \mathbf{Q}_{k} \mathbf{h}_{e_{k}}+\operatorname{tr}\left(\boldsymbol{\Gamma}_{e}^{2} \mathbf{Q}_{k}^{2}\right),
\end{aligned}
$$

where $\boldsymbol{\Gamma}_{e}=\mathbb{E}\left(\mathbf{e}_{i} \mathbf{e}_{i}^{H}\right)$ and is diagonal by assumption. Since each $\mathbf{Q}_{k}$ is linear in $\boldsymbol{\beta}$, the expression for the mean in (5.9) is linear in $\boldsymbol{\beta}$. Therefore, we can adapt the iterative linearization technique that was developed for the single case (Medra and Davidson, 2015a) to produce the following algorithm

1. Calculate the beamforming directions $\mathbf{u}_{k}$ using (5.6) and (5.7). 
2. Initialize $\sigma_{s_{k}}=1$.

3. Update $r^{\star}$, and $\left\{\beta_{k}\right\}$ using $\mathbb{E}\left(\mathbf{h}_{k}^{H} \mathbf{Q}_{k} \mathbf{h}_{k}-\sigma_{k}^{2}\right)=\sigma_{s_{k}} r^{\star}$ and $\sum_{k} \beta_{k}=P_{t}$, where the expected value is given in (5.9).

4. Update each $\sigma_{s_{k}}$ using (5.10).

5. Evaluate a termination criterion and return to 3 if not satisfied.

\subsubsection{Dominant per-base station power constraints}

When the constraint in (5.8c) is inactive, we can simplify the formulation in (5.8) to

$$
\begin{array}{rl}
r^{\star}=\max _{\mathbf{w}_{k}, r} & r \\
\text { s.t. } & \sum_{k=1}^{K} \mathbf{w}_{k}^{H} \boldsymbol{\Lambda}_{i} \mathbf{w}_{k} \leq P_{i}, \quad i=\{1,2, \ldots, B\}, \\
& \mathbf{h}_{e_{k}}^{H} \mathbf{Q}_{k} \mathbf{h}_{e_{k}}-\sigma_{k}^{2}-r \geq 0, \quad k=\{1,2, \ldots, K\} .
\end{array}
$$

As we will see, dealing with all the BSs together as a one virtual BS then applying perBS power constraints is analogous to dealing with one BS and applying per-antenna power constraints. Accordingly, the derivation for the closed-form solution for the problem in (5.11) will follow the same steps for the offset maximization algorithm with per-antenna power constraint (Yu and Lan, 2007; Medra and Davidson, 2015a). We will briefly summarize it here for completeness. Following what was done for the 
single case, we will consider the following equivalent problem

$$
\begin{array}{ll}
\min _{\mathbf{w}_{k}, \alpha} & \alpha \sum_{i=1}^{B} P_{i} \\
\text { s.t. } & \sum_{k=1}^{K} \mathbf{w}_{k}^{H} \boldsymbol{\Lambda}_{i} \mathbf{w}_{k} \leq \alpha P_{i}, \quad i=\{1,2, \ldots, B\}, \\
& \mathbf{h}_{e_{k}}^{H} \mathbf{Q}_{k} \mathbf{h}_{e_{k}}-\sigma_{k}^{2}-r^{\star} \geq 0, k=\{1,2, . ., K\} .
\end{array}
$$

The equivalence here means that both problems share an optimal solution. This can be verified by observing that substituting the optimal solution of (5.11) in (5.12) will give us a value of $\alpha=1$, and by observing that the optimal $\alpha$ can not be smaller than one, as that would mean that we could rescale the precoding vectors and have a larger $r^{\star}$ which contradicts the presumed optimality; i.e., problems (5.11) and (5.12) share the optimal solution with $\alpha=1$. If we define $q_{i}$ and $\nu_{i}$ to be the dual variables for the constraints in (5.12b) and (5.12c) respectively, and $\hat{\mathbf{Q}}=\sum_{i=1}^{B} q_{i} \boldsymbol{\Lambda}_{i}$, then we can write the Lagrangian of (5.12) as

$$
\begin{aligned}
\mathcal{L}\left(\mathbf{w}_{k}, \alpha, \nu_{k}, q_{i}\right)=\sum_{k=1}^{K} \nu_{k}\left(\sigma_{k}^{2}+r^{\star}\right)+\alpha\left(\sum_{i=1}^{B} P_{i}-\sum_{i=1}^{B} q_{i} P_{i}\right) & \\
& +\sum_{k=1}^{K} \mathbf{w}_{k}^{H}\left(\hat{\mathbf{Q}}+\sum_{j \neq k} \nu_{j} \mathbf{h}_{e_{j}} \mathbf{h}_{e_{j}}^{H}-\nu_{k} / \gamma_{k} \mathbf{h}_{e_{k}} \mathbf{h}_{e_{k}}^{H}\right) \mathbf{w}_{k} .
\end{aligned}
$$

The Lagrangian now has the same form as the Lagrangian in the case of one BS with per-antenna constraints presented by Medra and Davidson (2015b). Accordingly, we suggest using an analogous iterative quasi-closed form solution. In the case of equal $P_{i}$ that algorithm can be summarized as

1. Initialize $\hat{\mathbf{Q}}^{0}$ such that $\sum q_{i}=B$. Set $n=0$.

2. Find $\nu_{k}^{n}$ using the fixed point equations 


$$
\left(\nu_{k}^{n}\right)^{-1}=\mathbf{h}_{e_{k}}^{H}\left(\hat{\mathbf{Q}}^{n}+\sum_{j} \nu_{j}^{n} \mathbf{h}_{e_{j}} \mathbf{h}_{e_{j}}^{H}\right)^{-1} \mathbf{h}_{e_{k}}\left(1+1 / \gamma_{k}\right) .
$$

3. Solve for the directions $\mathbf{u}_{k}=\hat{\mathbf{w}}_{k} /\left\|\hat{\mathbf{w}}_{k}\right\|$, where

$$
\hat{\mathbf{w}}_{k}=\left(\hat{\mathbf{Q}}^{n}+\sum_{j} \nu_{j}^{n} \mathbf{h}_{e_{j}} \mathbf{h}_{e_{j}}^{H}\right)^{-1} \mathbf{h}_{e_{k}} .
$$

4. Find $r^{\star}$, and $\left\{\beta_{k}\right\}$ by solving $\mathbb{E}\left(\mathbf{h}_{k}^{H} \mathbf{Q}_{k} \mathbf{h}_{k}-\sigma_{k}^{2}\right)=\sigma_{s_{k}} r^{\star}$ and $\sum_{k} \beta_{k}=\sum_{i} P_{i}$.

5. Update $\hat{\mathbf{Q}}^{n+1}$ using $q_{i}^{n+1}=q_{i}^{n}+t_{n}\left(\sum_{k=1}^{K} \mathbf{w}_{k}^{H} \boldsymbol{\Lambda}_{i} \mathbf{w}_{k}-P_{i}\right)$, where $t_{n}$ is the step size used.

6. Increment $n$, check whether $\sum_{k=1}^{K} \mathbf{w}_{k}^{H} \boldsymbol{\Lambda}_{i} \mathbf{w}_{k}-P_{i}<\delta_{i}$, where $\delta_{i}$ is the maximum allowable violation of the power constraint for the $i$ th BS. If the test fails, return to 2 .

\subsection{Distributed algorithm based on virtual users}

Implementing the centralized processing and data sharing system described in the previous section can be a challenging task no matter how the beamformers and the power allocation are determined. Therefore, there is considerable interest in distributed systems that coordinate their signals via limited backhaul communications (Gesbert et al., 2010). In a distributed coordinated system each user is served by a single BS, and we will assume that that assignment has been made. We consider a system in which each BS obtains estimates of the channels to users that have been assigned to it, and also obtains estimates of the channels to users assigned to neighbouring BSs upon which the BS may impose significant interference. If we let $\mathbf{h}_{e_{k}}^{b}$ denote the estimate of the channel from BS $b$ to a user $k$ that is not assigned to that $\mathrm{BS}$, then one way in which BS $b$ could manage the interference it imposes on user 
$k$ would be to enforce a constraint of the form $\left\|\left(\mathbf{h}_{e_{k}}^{b}\right)^{H} \sum_{j} \mathbf{w}_{j}^{b}\right\|^{2}<\epsilon$. Such "softshaping" constraints (e.g., Scutari et al., 2008) are convex and can be incorporated into a variety of precoder design formulations (e.g., Hong and Luo, 2014; Scutari et al., 2008) and effective beamforming vectors optimization can be obtained using generic convex tools. However, the structure of those constraints results in dual formulations that do not appear to be amendable to the analysis that we developed for the centralized case. In our quest for low-complexity algorithms for the distributed case, we will instead consider an alternative design approach that takes into consideration the interference imposed on users in other cells by treating them as virtual users when designing the normalized beamformers in the cell of interest (Park and Lee, 2009; Lee and Shin, 2011; Mirza et al., 2015). This principle was implemented using a zero-forcing (ZF) approach by Park and Lee (2009); Lee and Shin (2011), and the regularized zero-forcing approach by Mirza et al. (2015). In this section we propose a scheme in which the normalized beamformers are designed using the closed-form solutions of the offset maximization approach in (5.6) and (5.7) and the power loading is designed using the simple iterative algorithm by Medra and Davidson (2015a).

To describe that approach, we let the set $\mathcal{K}_{i}$ denote the indices of the users assigned to BS $i$ and let $\tilde{\mathcal{K}}_{i}$ denote the union of that set and the indices of the users to which BS $i$ should mitigate its interference. We will discuss the selection of $\mathcal{K}_{i}$ and $\tilde{\mathcal{K}}_{i}$ below. The number of users in these sets are denoted by $K_{i}$ and $\tilde{K}_{i}$, respectively. With the goal of computation efficiency in mind, each BS designs the normalized beamformers as if it were designing them for all users in $\tilde{\mathcal{K}}_{i}$. It then designs the power loading for the users in $\mathcal{K}_{i}$. That procedure is as follows

1. Find $\nu_{k}$ for all users in $\tilde{\mathcal{K}}_{i}$ using (5.6). 
2. Solve for the directions for the users in $\mathcal{K}_{i}$ using (5.7).

3. Initialize $\sigma_{s_{k}}=1$.

4. Find the power loading for the users in $\mathcal{K}_{i}$ and $r^{\star}$ using (5.9) and the power constraint $\sum_{k \in \mathcal{K}_{i}} \beta_{k}=P_{i}$.

5. Update $\sigma_{s_{k}}$ using (5.10).

6. Return to 4 until an appropriate stopping criterion is satisfied.

Although the design of the beamforming directions and the power loading in the above algorithm is distributed, the BSs within a cluster coordinate their designs through the selection of the users in $\tilde{K}_{i}$. While many strategies are possible, one simple strategy that keeps the amount of information to be shared between the BSs in the cluster small is to first select $\mathcal{K}_{i}$ using a conventional BS assignment technique for cell-by-cell operation and then have the neighbouring BSs inform BS $i$ of the users in their cells that are to have interference mitigated. BS $i$ would acquire the CSI for these users as if they were assigned to the $i$ th cell.

The above algorithm provides implicit control over the influence that the users in $\tilde{K}_{i} \backslash \mathcal{K}_{i}$ have on the beamforming directions in cell $i$. This is provided through the target SINR for those virtual users. As can be seen from (5.6), $\nu_{k}$ scales in an approximately inverse fashion with $\gamma_{k}$, and as can be seen in (5.7), $\nu_{k}$ controls the influence of the channel to user $k$ on all the beamforming directions. This kind of flexibility is not present in the ZF and RZF techniques by Park and Lee (2009); Lee and Shin (2011); Mirza et al. (2015). 


\subsection{Simulation results}

To illustrate the performance of the proposed algorithms we consider a system consisting of two BSs separated by a distance of $2.4 \mathrm{~km}$, each with 4 antennas, serving a total of 4 users. Half of the users are uniformly distributed in a circle of radius $1.5 \mathrm{~km}$ around the first BS, the other half are similarly distributed around the other BS. We assume a large scale fading model described with a path-loss exponent of 3.52 and log-normal shadow fading with $8 \mathrm{~dB}$ standard deviation. The small scale fading is modelled using the standard i.i.d. Rayleigh model. We assume a TDD system with a channel estimation error variance $\sigma_{e}^{2}=0.04$, and an SINR target of $\gamma=3 \mathrm{~dB}$ for all users (including the "virtual users" in the algorithms of Section 5.5). The per-BS power constraint is $P_{i}=P_{t} K_{i} / K$. We assume that each user has a signal sensitivity of $-90 \mathrm{dBm}$, and we will consider this power as the noise power. In Fig. 5.1, we plot the outage probability versus the total power constraint $P_{t}$ for the cooperative algorithms with total power constraints (Section 5.4.1) and per-BS power constraints (Section 5.4.2). We also assess the performance of the distributed algorithms in Section 5.5; once with the users assigned to the BS for which they were generated and another time with BS selection according to the channel norm (i.e., the user is assigned to the BS that has a channel vector with bigger Euclidean norm).

We will compare our algorithms to two centralized algorithms and one distributed algorithm from the literature. The first is an adaptation of the robust centralized coordination algorithm by Pennanen et al. (2014, Equation (5)) for the considered scenario. In the adaptation a binary search on the zero-outage region size is performed to find the largest "zero-outage" region for which a problem with a total power constraint is feasible. That problem is convex, but involves many linear matrix 
inequality constraints. The second comparison is with the robust centralized coordination algorithm by Qiu et al. (2010, Equation (5)) with per-BS power constraint $P_{i}=P_{t} / 2$. This algorithm involves repeated solutions of modified perfect-CSI problems. In these two cases, each BS is assigned to the users in its area (no BS selection), and the processing is done in a centralized manner.

From Fig. 5.1 we observe, as expected, that the centralized cooperative algorithms proposed in Sections 5.4.1 and 5.4.2 provide the best performance. Perhaps the more interesting observations from Fig. 5.1 are that the proposed distributed coordination algorithm provides better performance than the existing centralized coordination algorithms by Pennanen et al. (2014) and Qiu et al. (2010). This is despite the fact that the centralized algorithms by Pennanen et al. (2014) and Qiu et al. (2010) incur significantly larger computational costs. A comparison of the distributed algorithms in Fig. 5.1 shows that the proposed distributed coordination algorithm outperforms the original versions of the distributed coordination RZF-based algorithm by Mirza et al. (2015) that uses the regularization factor described by Mirza et al. (2015, Equation (52)). Furthermore, when the proposed algorithm is augmented with a simple BS selection scheme it also outperforms a variant of the algorithm by Mirza et al. (2015) that employs the power loading developed by Medra and Davidson (2015a). (That variant significantly improves the performance of Mirza et al., 2015). This is despite the fact that the proposed algorithm is based on a simple iterative algorithm. For reference, Fig. 5.1 includes the performance of the power loaded offset maximization approach (Medra et al., 2015) applied to each cell individually. In the low power regime this approach performs well, whereas at higher power levels, where the impact of the interference increases, its relative performance degrades. 


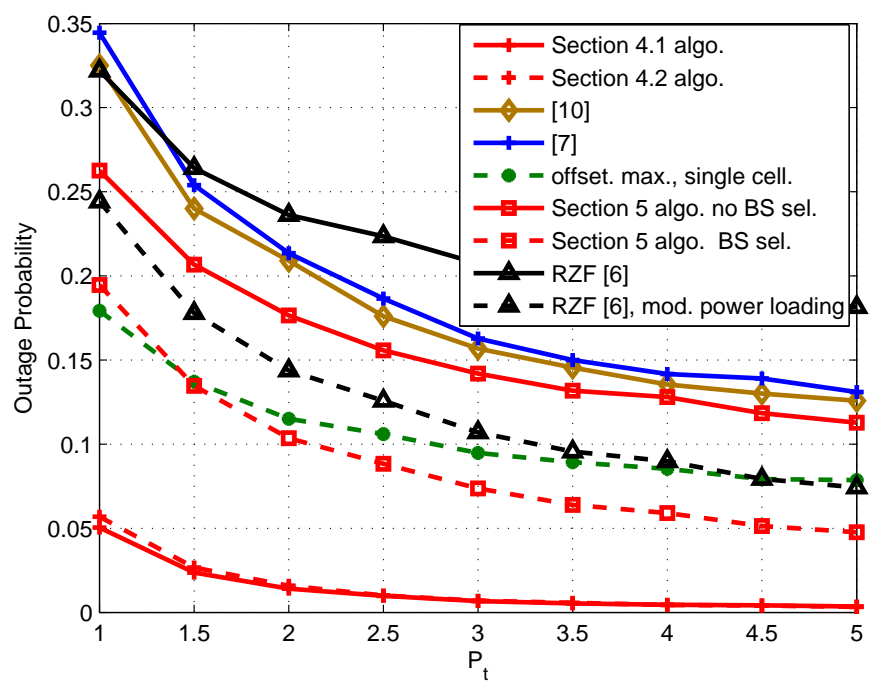

Figure 5.1: Outage probability for 4 users, 4 antennas, $\gamma=3 \mathrm{~dB}, \sigma^{2}=-90 \mathrm{dBm}, \sigma_{h}=0.2$. Here [6] refers to Mirza et al. (2015), [7] refers to Qiu et al. (2010), and [10] refers to Pennanen et al. (2014).

\subsection{Conclusion}

In this paper we proposed multi-cell MISO downlink algorithms that can provide substantial robustness against channel uncertainties. We proposed a centralized cooperative algorithm that has significant performance gains compared to other algorithms in literature, and has lower computational complexity. We also provided a distributed coordination algorithm that needs very limited backhaul communication, and incurs an even lower computational cost, and yet can provide better performance than several existing methods. 


\section{Chapter 6}

\section{Summary, conclusions and future work}

\subsection{Summary}

This thesis focused on the design of low-complexity robust linear beamforming algorithms for multi-user downlink multiple-input single-output (MISO) communication systems. The algorithms were designed either to provide certain SINR levels with prespecified probabilities using the least possible transmitted power or to minimize the outage probability under certain power constraints.

Chapter 2 introduced a zero-outage region approach for robust downlink beamforming in frequency division duplexing (FDD) systems. By analyzing the structure of the semidefinite relaxation of the design problem, a scalar approximation of the linear matrix inequalities (LMIs) was developed and this led to a low-complexity algorithm that provides a balance between the performance inside and outside the 
zero-outage region. This approach was shown to extend naturally to time division duplexing (TDD) systems. Further analysis of the approximation of the LMIs revealed that the proposed approach implicitly generates an offset-style approximation of the SINR outage constraint. That offset-based approach plays a key role in this thesis. That interpretation motivated the work in Chapter 3, where improved offset-based approximations for the SINR outage constraint were introduced, and low-complexity algorithms were derived. A near-optimal robust power loading technique was also introduced. The beamformers designed using the offset maximization framework were shown to provide significant performance gains and to possess several design flexibilities, in addition to being of low complexity.

In Chapter 4, per-antenna power constraints (PAPCs) were introduced to the offset maximization framework. Consistent with the theme of the thesis, the suggested algorithms enable the management of the outage probability at low computational cost, thus allowing the usability in different applications. Beyond the introduction of PAPCs to the offset-maximization directions, the PAPCed variants of conventional beamforming methods, such as zero forcing (ZF) and maximum ratio transmission (MRT) were also developed. When the number of antennas is large, the computational cost of the MRT-based algorithm grows only linearly in the number of antennas, making it suitable for massive MISO systems.

The previous discussion focused on intra-cell interference, and the interference from other cells was assumed to be managed independently. In Chapter 5, the offset maximization algorithm was extended in the multi-cell case and provided joint robustness against inter-cell and intra-cell interferences. The proposed multi-cell algorithms provide a trade-off between the amount of cooperation or coordination and 
the outage performance.

\subsection{Conclusions}

This thesis has presented a suite of algorithms that provide high-quality approximate solutions to a broad range of robust downlink beamforming problems with quality-ofservice constraints. The unifying feature of these algorithms is that they are based, either explicitly or implicitly, on a transformation of each SINR outage constraint into a non-negativity constraint on a random variable, and the approximation of that non-negativity constraint by various forms of "offset" on the mean of the distribution. It was shown that this approach can generate robust beamformers that provide significant performance gains, and that it can do so at low computational cost. A feature of the offset-based approach is that it facilitates the separation of the design of the beamforming directions and the power loading; providing computationally cheap algorithms for the directions and a near-optimal algorithm for the power loading. The approach is also quite flexible, as demonstrated by its ability to accommodate different power constraints, including the PAPCs and the total power constraints. That flexibility was further demonstrated by the extension to multi-cell environments in which intra-cell interference is managed in addition to the inter-cell interference. Moreover, the offset maximization framework provides significant insights into the trade-off between power consumption and outage probability. Perhaps most importantly, the family of the offset maximization algorithms was shown to admit further approximations that result in even lower computational cost. That enables the application of the approach to envisioned systems with a large number of antennas. Accordingly, this thesis suggests the use of offset maximization principles 
to tackle the channel uncertainties in downlink beamforming problems, rather than the conservative zero-outage region-based ideas that are abundant in the existing literature.

\subsection{Future work}

This thesis presents the offset maximization technique as a framework for robust downlink precoding, and provides many problems that can be efficiently solved using this framework. However, there are several additional design challenges that could benefit from being viewed within that framework.

1. The work done in this thesis focused on quality-of-service (QoS) kind of problems, where users specify the required SINR targets for proper operations. A related problem of interest is that of maximizing the sum rate of the system. The extension of the low-complexity offset maximization framework to maximize the sum rate in the presence of channel uncertainty appears to be worthy of investigation. When the quality of the channel state information (CSI) at the BS scales linearly with the signal-to-noise (SNR), the slope of the sum rate curve (versus the SNR, in decibels) can be the same as the perfect CSI case (Jindal, 2006). However, the curve will have an offset which might be reduced using the proposed framework. More improvements are possible when the CSI quality is kept constant or when the quality does not improve linearly in the SNR. When the system is narrow-band, the sum rate maximization might not provide each user with the required rate for proper operation, and weighted versions of the 
sum rate maximization can be used. When the system is wide-band, and orthogonal frequency division multiplexing (OFDM) is used to transform the wide frequency selective channel to many flat fading channels, then maximizing the sum rate can be beneficial in terms that each user is provided higher rate, on average, on different frequency bins. However, the large scale fading of the user channel can cause the far users to have lower rates on all the allocated frequency bins, in which case, some sort of fairness control should be introduced.

2. The PAPCs in Chapter 4 were designed for a narrow-band system. In many practical applications, the system is typically a wide-band system that uses OFDM techniques, or variants thereof, to provide multiple narrow bands that have flat fading instead of frequency selective fading. The OFDM block does not change the input signal power, however, it suffers from high peak to average power ratio (PAPR). The PAPR is a well known problem in OFDM literature, and significant efforts were done to reduce that ratio (e.g., Rahmatallah and Mohan, 2013). However, the literature in the case of beamforming is not as rich (e.g., Arvola et al., 2016; Joung et al., 2007). The extension of the offset maximization framework to accommodate for PAPCs in the case of OFDM signalling while providing low PAPR is interesting. Since the offset-based power loading algorithm provides explicit relationship between the power required for each user and the outage probability of that user, this relationship might be able to be used to suggest some sort of power allocation over the different frequency bins. This power allocation can be designed to reduce the PAPR.

3. The channel modelling in this thesis was represented by small and large scale fading. The small scale fading was described by Rayleigh fading channel, with 
the channel from each BS antenna to each user being modelled as independent with a circular complex Gaussian distribution with zero mean and unit variance. The large scale fading is described by a path-loss exponent and lognormal shadow fading. The channel uncertainty models discussed are that of additive Gaussian errors that typically appear in time division duplexing (TDD) systems, and that arise from downlink training and quantized feedback in frequency division duplexing (FDD) systems. Recently, there has been great interest in using the millimeter waves as it has plenty of available spectrum in that frequency range. The channel models of the millimeter waves are different from those of conventional microwave links, in terms of the parameters of large scale fading, and the nature of the small scale fading. The small scale fading was measured by Samimi et al. (2016) and shown to be rather Rician than Rayleigh even in the non line-of-sight case. The high attenuation nature of the millimeter waves provide channel matrices that might be sparse (e.g., Sun et al., 2014). The adaptation of the offset maximization for that frequency range can be of great importance.

4. The offset maximization framework relied on having channel estimates to produce low-complexity robust beamforming. Those channel estimates rely on the small scale and large scale fading. The large scale fading typically changes very slowly as it depends on the distance between the base station (BS) and the user, whereas, the small scale fading can change very frequently. The application of offset maximization to produce beamforming vectors that are based on large scale fading only would allow those vectors to be used for many time blocks. While there might be some performance degradation due to using the 
large scale fading only, the channel estimation will be required less frequently and the overall degradation might be negligible.

5. In this thesis, the users are assumed to have single antenna. When the user has multiple antennas, that opens the opportunity to improve the performance in two different ways. The BS can send multiple data streams to that user, and the user can apply a multiple-input multiple output (MIMO) detector to regenerate the transmitted symbols. However, the significant complexity of the optimal detector suggests the use of simpler sub-optimal linear detectors. Using such a spatial multiplexing strategy can provide higher rates to the user. Another possible strategy is using the multiple antennas at the BS to send the same data to the user. When using spatial diversity, the reliability and the resulting SINR can be improved by designing a receiver beamforming vector at the user side. While single-antenna users require beamforming processing at the BS side, in multi-antenna users case, detection algorithms should be deployed at the user side as well. In the later case, the low-complexity algorithms are more important as the computational power of the user equipment is significantly lower than the BS.

6. In this thesis the multi-cell case were addressed according to two different scenarios. The cooperative scenario, and the coordinated distributed scenario. Another network architecture that might be of interest is that coordinated centralized scheme, where each user is assigned to one BS only, and the beamforming design for the users is done jointly so that the inter-cell and intra-cell interferences are minimized. The adaptation of offset maximization to this case may be of considerable interest. 


\section{Bibliography}

Al-Naffouri, T. Y., Moinuddin, M., Ajeeb, N., Hassibi, B., and Moustakas, A. L. (2016). On the distribution of indefinite quadratic forms in Gaussian random variables. IEEE Trans. Commun., 64(1), 153-165.

Alodeh, M., Chatzinotas, S., and Ottersten, B. (2015). Constructive multiuser interference in symbol level precoding for the MISO downlink channel. IEEE Trans. Signal Process., 63(9), 2239-2252.

Alodeh, M., Spano, D., Kalantari, A., Tsinos, C., Christopoulos, D., Chatzinotas, S., and Ottersten, B. (2017). Symbol-level and multicast precoding for multiuser multiantenna downlink: A survey, classification and challenges. arXiv preprint arXiv:1703.0361\%.

Arvola, A., Tolli, A., and Gesbert, D. (2016). A user cooperative beamforming approach to PAPR reduction in MIMO-OFDM uplink. In Conf. Rec. 50th Asilomar Conf. Signals, Systems, Computers, pages 691-695.

Ben-Tal, A. and Nemirovski, A. (2001). Lectures on Modern Convex Optimization: Analysis, Algorithms, and Engineering Applications. SIAM. 
Ben-Tal, A., El Ghaoui, L., and Nemirovski, A. (2009). Robust optimization. Princeton University Press.

Bengtsson, M. and Ottersten, B. (2001). Optimal and suboptimal transmit beamforming. In L. C. Godara, editor, Handbook of Antennas in Wireless Communications, chapter 18. CRC Press.

Bjornson, E., Bengtsson, M., and Ottersten, B. (2014). Optimal multiuser transmit beamforming: A difficult problem with a simple solution structure. IEEE Signal Process. Mag., 31(4), 142-148.

Boyd, S. and Vandenberghe, L. (2004). Convex Optimization. Cambridge University Press.

Caire, G., Jindal, N., Kobayashi, M., and Ravindran, N. (2010). Multiuser MIMO achievable rates with downlink training and channel state feedback. 56(6), 28452866.

Chang, T.-H., Ma, W.-K., and Chi, C.-Y. (2011). Worst-case robust multiuser transmit beamforming using semidefinite relaxation: Duality and implications. In Conf. Rec. 45th Asilomar Conf. Signals, Systems, Computers, pages 1579-1583, Pacific Grove, CA.

Cisco (2016). Cisco visual networking index: Global mobile data traffic forecast update, 2016-2021. White Paper. Available at http://www.cisco.com.

Costa, M. (1983). Writing on dirty paper (corresp.). IEEE Trans. Inf. Theory, 29(3), 439-441. 
Dahrouj, H. and Yu, W. (2010). Coordinated beamforming for the multicell multiantenna wireless system. IEEE Trans. Wireless Commun., 9(5), 1748-1759.

Dartmann, G., Gong, X., Afzal, W., and Ascheid, G. (2013). On the duality of the maxmin beamforming problem with per-antenna and per-antenna-array power constraints. IEEE Trans. Veh. Tech., 62(2), 606-619.

Ding, P., Love, D. J., and Zoltowski, M. D. (2007). Multiple antenna broadcast channels with shape feedback and limited feedback. IEEE Trans. Signal Process., 55(7), 3417-3428.

Feng, C. and Jing, Y. (2016). Modified MRT and outage probability analysis for massive MIMO downlink under per-antenna power constraint. In Proc. IEEE Int. Wkshp Signal Process. Adv. Wireless Commun., pages 1-6.

Gershman, A. B., Sidiropoulos, N. D., ShahbazPanahi, S., Bengtsson, M., and Ottersten, B. (2010). Convex optimization-based beamforming. IEEE Signal Process. Mag., 27(3), 62-75.

Gesbert, D., Kountouris, M., Heath, R. W., Chae, C.-B., and Salzer, T. (2007). Shifting the MIMO paradigm. IEEE Signal Proc. Mag., 24(5), 36-46.

Gesbert, D., Hanly, S., Huang, H., Shamai Shitz, S., Simeone, O., and Yu, W. (2010). Multi-cell MIMO cooperative networks: A new look at interference. IEEE J. Sel. Areas Commun., 28(9), 1380-1408.

Goldsmith, A., Jafar, S. A., Jindal, N., and Vishwanath, S. (2003). Capacity limits of MIMO channels. IEEE J. Sel. Areas Commun., 21(5), 684-702. 
Grant, M., Boyd, S., and Ye, Y. (2008). CVX: Matlab software for disciplined convex programming.

He, X. and Wu, Y.-C. (2013). Probabilistic QoS constrained robust downlink multiuser MIMO transceiver design with arbitrarily distributed channel uncertainty. IEEE Trans. Wireless Commun., 12(12), 6292-6302.

Hochwald, B. M., Marzetta, T. L., and Tarokh, V. (2004). Multiple-antenna channel hardening and its implications for rate feedback and scheduling. IEEE Trans. Inf. Theory, 50(9), 1893-1909.

Hochwald, B. M., Peel, C. B., and Swindlehurst, A. L. (2005). A vector-perturbation technique for near-capacity multiantenna multiuser communication-part II: perturbation. IEEE Trans. Commun., 53(3), 537-544.

Hong, M. and Luo, Z.-Q. (2014). Signal processing and optimal resource allocation for the interference channel. In N. D. Sidiropoulos, F. Gini, R. Chellappa, and S. Theodoridis, editors, Academic Press Library in Signal Processing: Volume 2 Communications and Radar Signal Processing, volume 2, chapter 8, pages 409 469. Elsevier.

Huang, Y. and Palomar, D. P. (2010). Rank-Constrained separable semidefinite programming with applications to optimal beamforming. IEEE Trans. Signal Process., 58(2), 664-678.

Huang, Y. and Palomar, D. P. (2014). Randomized algorithms for optimal solutions of double-sided QCQP with applications in signal processing. IEEE Trans. Signal Process., 62(5), 1093-1108. 
Huang, Y. and Zhang, S. (2007). Complex matrix decomposition and quadratic programming. Math. Oper. Res., 32(3), 758-768.

Huang, Y., Palomar, D. P., and Zhang, S. (2013). Lorentz-positive maps and quadratic matrix inequalities with applications to robust MISO transmit beamforming. IEEE Trans. Signal Process., 61(5), 1121-1130.

Jindal, N. (2006). MIMO broadcast channels with finite-rate feedback. IEEE Trans. Inf. Theory, 52(11), 5045-5060.

Joham, M., Castro, P., Utschick, W., and Castedo, L. (2012). Robust precoding with limited feedback design based on precoding MSE for MU-MISO systems. IEEE Trans. Signal Process., 60(6), 3101-3111.

Joudeh, H. and Clerckx, B. (2016). Robust transmission in downlink multiuser MISO systems: A rate-splitting approach. IEEE Trans. Signal Process., 64(23), 62276242.

Joung, J., Jeong, E. R., and Lee, Y. H. (2007). Beamforming and PAPR reduction for MISO-OFDM systems. In Proc. IEEE Int. Conf. Acoustics, Speech, Signal Process., volume 3, pages III-377-III-380.

Kaltenberger, F., Jiang, H., Guillaud, M., and Knopp, R. (2010). Relative channel reciprocity calibration in MIMO/TDD systems. In Proc. Future Network and Mobile Summit, 2010, pages 1-10.

Kandukuri, S. and Boyd, S. (2002). Optimal power control in interference-limited fading wireless channels with outage-probability specifications. IEEE Trans. Wireless Commun., 1(1), 46-55. 
Lee, N. and Shin, W. (2011). Adaptive feedback scheme on K-cell MISO interfering broadcast channel with limited feedback. IEEE Trans. Wireless Commun., 10(2), 401-406.

Liu, T. and Chen, J. (2009). Joint downlink transmit and receive beamforming under per-antenna power constraints. In Proc. 7th Int. Conf. Inf. Commun. Signal Proc., pages $1-5$.

Lo, T. K. Y. (1999). Maximum ratio transmission. IEEE Trans. Commun., 47(10), $1458-1461$.

Love, D. J., Heath, R. W., and Strohmer, T. (2003). Grassmannian beamforming for multiple-input multiple-output wireless systems. 49(10), 2735-2747.

Love, D. J., Heath, R. W., Lau, V. K. N., Gesbert, D., Rao, B. D., and Andrews, M. (2008). An overview of limited feedback in wireless communication systems. IEEE J. Sel. Areas Commun., 26(8), 1341-1365.

Luo, Z.-Q., Ma, W.-K., So, A. M.-C., Ye, Y., and Zhang, S. (2010). Semidefinite relaxation of quadratic optimization problems. 27(3), 20-34.

Ma, W. K., Pan, J., So, A. M. C., and Chang, T. H. (2017). Unraveling the rankone solution mystery of robust MISO downlink transmit optimization: A verifiable sufficient condition via a new duality result. IEEE Trans. Signal Process., 65(7), 1909-1924.

Medra, M. and Davidson, T. N. (2015a). Per-user outage-constrained power loading technique for robust MISO downlink. In Conf. Rec. 49th Asilomar Conf. Signals, Systems, Computers, pages 1232-1236. 
Medra, M. and Davidson, T. N. (2015b). Robust MISO downlink precoder design with per-antenna power constraints. In Proc. IEEE Int. Wkshp Signal Process. Adv. Wireless Commun., pages 580-584, Stockholm.

Medra, M. and Davidson, T. N. (2016). Robust MISO downlink: An efficient algorithm for improved beamforming directions. In Proc. IEEE Signal Process. Wkshp Sensor Array and Multichannel Signal Process., pages 1-5.

Medra, M., Ma, W.-K., and Davidson, T. N. (2015). Low-complexity robust MISO downlink precoder optimization for the limited feedback case. In Proc. IEEE Int. Conf. Acoustics, Speech, Signal Process., pages 3108-3112, Brisbane.

Medra, M., Huang, Y., Ma, W. K., and Davidson, T. N. (2016). Low-complexity robust MISO downlink precoder design under imperfect CSI. IEEE Trans. Signal Process., 64(12), 3237-3249.

Mirza, J., Smith, P. J., Dmochowski, P. A., and Shafi, M. (2015). Coordinated regularized zero-forcing precoding for multicell MISO systems with limited feedback. arXiv preprint arXiv:1508.07333.

Park, S.-H. and Lee, I. (2009). Analysis of degrees of freedom of interfering MISO broadcast channels. In Proc. IEEE Global Telecommun. Conf., pages 1-6.

Pascual-Iserte, A., Palomar, D., Perez-Neira, A., and Lagunas, M. (2006). A robust maxmin approach for MIMO communications with imperfect channel channel state information based on convex optimization. 45, 346-360.

Peel, C., Hochwald, B., and Swindlehurst, A. (2005). A vector-perturbation technique 
for near-capacity multiantenna multiuser communication-Part I: Channel inversion and regularization. IEEE Trans. Commun., 53(1), 195-202.

Pennanen, H., Tolli, A., and Latva-aho, M. (2011). Decentralized coordinated downlink beamforming via primal decomposition. IEEE Signal Process. Letters, 18(11), $647-650$.

Pennanen, H., Tolli, A., and Latva-aho, M. (2014). Decentralized robust beamforming for coordinated multi-cell MISO networks. IEEE Signal Process. Letters, 21(3), 334-338.

Pólik, I. and Terlaky, T. (2007). A survey of the S-lemma. SIAM Review, 49(3), $371-418$.

Poor, H. V. (1994). An Introduction to Signal Detection and Estimation. Springer.

Qiu, J., Zhang, R., Luo, Z.-Q., and Cui, S. (2010). Optimal distributed beamforming for MISO interference channels. In Conf. Rec. 44th Asilomar Conf. Signals, Systems, Computers, pages 277-281.

Rahmatallah, Y. and Mohan, S. (2013). Peak-to-average power ratio reduction in OFDM systems: A survey and taxonomy. IEEE Commun. Surveys Tutorials, 15(4), $1567-1592$.

Rashid-Farrokhi, F., Tassiulas, L., and Liu, K. J. R. (1998a). Joint optimal power control and beamforming in wireless networks using antenna arrays. IEEE Trans. Commun., 46(10), 1313-1324.

Rashid-Farrokhi, F., Liu, K. J. R., and Tassiulas, L. (1998b). Transmit beamforming 
and power control for cellular wireless systems. IEEE J. Sel. Areas Commun., 16(8), 1437-1450.

Samimi, M. K., MacCartney, G. R., Sun, S., and Rappaport, T. S. (2016). 28 GHz millimeter-wave ultrawideband small-scale fading models in wireless channels. In 2016 IEEE 83rd Vehicular Technology Conference (VTC Spring), pages 1-6.

Schubert, M. and Boche, H. (2004). Solution of the multiuser downlink beamforming problem with individual SINR constraints. IEEE Trans. Veh. Tech., 53(1), 18-28.

Schubert, M. and Boche, H. (2005). Iterative multiuser uplink and downlink beamforming under SINR constraints. IEEE Trans. Signal Process., 53(7), 2324-2334.

Schubert, M. and Boche, H. (2007). A generic approach to QoS-based transceiver optimization. IEEE Trans. Commun., 55(8), 1557-1566.

Scutari, G., Palomar, D. P., and Barbarossa, S. (2008). Cognitive MIMO radio. IEEE Signal Process. Mag., 25(6), 46-59.

Scutari, G., Facchinei, F., Song, P., Palomar, D. P., and Pang, J. S. (2014). Decomposition by partial linearization: Parallel optimization of multi-agent systems. IEEE Trans. Signal Process., 62(3), 641-656.

Shen, C., Chang, T.-H., Wang, K.-Y., Qiu, Z., and Chi, C.-Y. (2012a). Chanceconstrained robust beamforming for multi-cell coordinated downlink. In Proc. IEEE Global Commun. Conf., pages 4957-4962.

Shen, C., Chang, T.-H., Wang, K.-Y., Qiu, Z., and Chi, C.-Y. (2012b). Distributed robust multicell coordinated beamforming with imperfect CSI: An ADMM approach. IEEE Trans. Signal Process., 60(6), 2988-3003. 
Shen, S. and Lok, T.-M. (2014). Downlink beamforming and power control with perantenna power constraints. In Proc. IEEE Wireless Commun. Networking Conf., pages 1206-1211.

Shenouda, M. B. and Davidson, T. N. (2007). Convex conic formulations of robust downlink precoder designs with quality of service constraints. IEEE J. Sel. Topics Signal Process., 1(4), 714-724.

Shenouda, M. B. and Davidson, T. N. (2008a). On the design of linear transceivers for multiuser systems with channel uncertainty. IEEE J. Sel. Areas Commun., 26(6), $1015-1024$.

Shenouda, M. B. and Davidson, T. N. (2008b). Probabilistically-constrained approaches to the design of the multiple antenna downlink. In Conf. Rec. 42nd Asilomar Conf. Signals, Systems, Computers, pages 1120-1124, Pacific Grove, CA.

Shenouda, M. B. and Davidson, T. N. (2009). Nonlinear and linear broadcasting with QoS requirements: Tractable approaches for bounded channel uncertainties. IEEE Trans. Signal Process., 57(5), 1936-1947.

Sohrabi, F. and Davidson, T. N. (2016). Coordinate update algorithms for robust power loading for the MU-MISO downlink with outage constraints. IEEE Trans. Signal Process., 64(11), 2761-2773.

Song, E., Shi, Q., Sanjabi, M., Sun, R.-Y., and Luo, Z.-Q. (2012). Robust SINRconstrained MISO downlink beamforming: When is semidefinite programming relaxation tight? EURASIP J. Wireless Commun. Networking, 1, 1-11. 
Spencer, Q. H., Swindlehurst, A. L., and Haardt, M. (2004). Zero-forcing methods for downlink spatial multiplexing in multiuser MIMO channels. 52(2), 461-471.

Sturm, J. (1999). Using SeDuMi 1.02, a MATLAB toolbox for optimization over symmetric cones. Optimization Methods and Software, 11-12, 625-653.

Sturm, J. F. and Zhang, S. (2003). On cones of nonnegative quadratic functions. Math. Oper. Res., 28(2), 246-267.

Sun, S., Rappaport, T. S., Heath, R. W., Nix, A., and Rangan, S. (2014). MIMO for millimeter-wave wireless communications: Beamforming, spatial multiplexing, or both? IEEE Commun. Mag., 52(12), 110-121.

Tajer, A., Prasad, N., and Wang, X. (2011). Robust linear precoder design for multicell downlink transmission. IEEE Trans. Signal Process., 59(1), 235-251.

Tolli, A., Pennanen, H., and Komulainen, P. (2011). Decentralized minimum power multi-cell beamforming with limited backhaul signaling. IEEE Trans. Wireless Commun., 10(2), 570-580.

Tseng, F.-S. and Gu, J.-F. (2015). Robust beamforming design in MISO interference channels with RVQ limited feedback. IEEE Trans. Veh. Technol., 64(2), 580-592.

Tshangini, M. and Nakhai, M. R. (2013). Second-order cone programming for robust downlink beamforming with imperfect CSI. In Proc.IEEE Global Commun. Conf., pages $3452-3457$.

Ubaidulla, P. and Chockalingam, A. (2008). Precoder designs for MIMO broadcast channels with imperfect CSI. In IEEE Int. Conf. Wireless, Mobile Computing, Networking, Commun., pages 145-150. 
Vucic, N. and Boche, H. (2007). Robust transceiver optimization for multiuser MISO broadcast systems with MSE targets. In Proc. 2nd IEEE Int. Wkshp on Computational Adv. in Multi-Sensor Adaptive Processing, pages 73-76.

Vucic, N. and Boche, H. (2009a). Robust QoS-constrained optimization of downlink multiuser MISO systems. IEEE Trans. Signal Process., 57(2), 714-725.

Vucic, N. and Boche, H. (2009b). A tractable method for chance-constrained power control in downlink multiuser MISO systems with channel uncertainty. IEEE Signal Proc. Letters, 16(5), 346-349.

Wang, K.-Y., So, A.-C., Chang, T.-H., Ma, W.-K., and Chi, C.-Y. (2014). Outage constrained robust transmit optimization for multiuser MISO downlinks: Tractable approximations by conic optimization. IEEE Trans. Signal Process., 62(21), 56905705.

Weingarten, H., Steinberg, Y., and Shamai, S. (2006). The capacity region of the Gaussian multiple-input multiple-output broadcast channel. 52(9), 3936-3964.

Wiesel, A., Eldar, Y., and Shamai, S. (2006). Linear precoding via conic optimization for fixed MIMO receivers. 54(1), 161-176.

Wiesel, A., Eldar, Y. C., and Shamai, S. (2008). Zero-forcing precoding and generalized inverses. IEEE Trans. Signal Process., 56(9), 4409-4418.

Windpassinger, C., Fischer, R. F. H., and Huber, J. B. (2004a). Lattice-reductionaided broadcast precoding. IEEE Trans. Commun., 52(12), 2057-2060.

Windpassinger, C., Fischer, R. F. H., Vencel, T., and Huber, J. B. (2004b). Precoding 
in multiantenna and multiuser communications. IEEE Trans. Wireless Commun., 3(4), 1305-1316.

Yazarel, Y. K. and Aktas, D. (2007). Downlink beamforming under individual SINR and per antenna power constraints. In Proc. IEEE Pac. Rim Conf. Commun. Comput. Signal Process., pages 422-425.

Ye, Y. (2011). Interior point algorithms: Theory and Analysis, volume 44. John Wiley \& Sons.

Yoo, T. and Goldsmith, A. (2006). On the optimality of multiantenna broadcast scheduling using zero-forcing beamforming. IEEE J. Sel. Topics Signal Process., 24(3), 528-541.

Yu, W. and Lan, T. (2007). Transmitter optimization for the multi-antenna downlink with per-antenna power constraints. 55(6), 2646-2660.

Zhang, Q., He, C., and Jiang, L. (2015). Per-stream MSE based linear transceiver design for MIMO interference channels with CSI error. IEEE Trans. Commun., 63(5), 1676-1689.

Zheng, G., Wong, K.-K., and Ng, T.-S. (2008). Robust linear MIMO in the downlink: A worst-case optimization with ellipsoidal uncertainty regions. EURASIP J. Adv. Signal Process., 2008, 154:1-154:15.

Zheng, G., Ma, S., Wong, K.-K., and Ng, T.-S. (2009). Robust beamforming in the MISO downlink with quadratic channel estimation and optimal training. IEEE Trans. Wireless Commun., 8(3), 1067-1072. 